\title{
Event generator tunes obtained from underlying event and multiparton scattering measurements
}

\author{
CMS Collaboration* \\ CERN, 1211 Geneva 23, Switzerland
}

Received: 2 December 2015 / Accepted: 29 February 2016 / Published online: 17 March 2016

(c) CERN for the benefit of the CMS collaboration 2016. This article is published with open access at Springerlink.com

\begin{abstract}
New sets of parameters ("tunes") for the underlying-event (UE) modelling of the PYTHIA8, PYTHIA6 and HERWIG++ Monte Carlo event generators are constructed using different parton distribution functions. Combined fits to CMS UE proton-proton (pp) data at $\sqrt{s}=7 \mathrm{TeV}$ and to UE proton-antiproton ( $\mathrm{p} \overline{\mathrm{p}}$ ) data from the CDF experiment at lower $\sqrt{s}$, are used to study the UE models and constrain their parameters, providing thereby improved predictions for proton-proton collisions at $13 \mathrm{TeV}$. In addition, it is investigated whether the values of the parameters obtained from fits to UE observables are consistent with the values determined from fitting observables sensitive to double-parton scattering processes. Finally, comparisons are presented of the UE tunes to "minimum bias" (MB) events, multijet, and DrellYan ( $\mathrm{q} \overline{\mathrm{q}} \rightarrow \mathrm{Z} / \gamma^{*} \rightarrow$ lepton-antilepton+jets) observables at 7 and $8 \mathrm{TeV}$, as well as predictions for MB and UE observables at $13 \mathrm{TeV}$.
\end{abstract}

\section{Introduction}

Monte Carlo (MC) event generators of hadron-hadron collisions based on perturbative quantum chromodynamics (QCD) contain several components. The "hard-scattering" part of the event consists of particles resulting from the hadronization of the two partons (jets) produced in the hardest scattering, and in their associated hard initial- and finalstate radiation (ISR and FSR). The underlying event (UE) consists of particles from the hadronization of beam-beam remnants (BBR), of multiple-parton interactions (MPI), and their associated ISR and FSR. The BBR include hadrons from the fragmentation of spectator partons that do not exchange any appreciable transverse momentum $\left(p_{\mathrm{T}}\right)$ in the collision. The MPI are additional 2-to-2 parton-parton scatterings that occur within the same hadron-hadron collision, and are softer in transverse momentum $\left(p_{\mathrm{T}} \lesssim 3 \mathrm{GeV}\right)$ than the hard scattering.

\footnotetext{
*e-mail: cms-publication-committee-chair@cern.ch
}

The perturbative 2-to-2 parton-parton differential cross section diverges like $1 / \hat{p}_{\mathrm{T}}^{4}$, where $\hat{p}_{\mathrm{T}}$ is the transverse momentum of the outgoing partons in the parton-parton center-of-mass (c.m.) frame. Usually, QCD MC models such as PYTHIA [1-5] regulate this divergence by including a smooth phenomenological cutoff $p_{\mathrm{T} 0}$ as follows:

$1 / \hat{p}_{\mathrm{T}}^{4} \rightarrow 1 /\left(\hat{p}_{\mathrm{T}}^{2}+p_{\mathrm{T} 0}^{2}\right)^{2}$.

This formula approaches the perturbative result for large scales and is finite as $\hat{p}_{\mathrm{T}} \rightarrow 0$. The divergence of the strong coupling $\alpha_{\mathrm{S}}$ at low $\hat{p}_{\mathrm{T}}$ is also regulated through Eq. (1). The primary hard 2-to-2 parton-parton scattering process and the MPI are regulated in the same way through a single $p_{\mathrm{T} 0}$ parameter. However, this cutoff is expected to have a dependence on the center-of-mass energy of the hadron-hadron collision $\sqrt{s}$. In the PYTHIA MC event generator this energy dependence is parametrized with a power-law function with exponent $\epsilon$ :

$p_{\mathrm{T} 0}(\sqrt{s})=p_{\mathrm{T} 0}^{\mathrm{ref}}\left(\sqrt{s} / \sqrt{s_{0}}\right)^{\epsilon}$,

where $\sqrt{s_{0}}$ is a given reference energy and $p_{\mathrm{T} 0}^{\text {ref }}$ is the value of $p_{\mathrm{T} 0}$ at $\sqrt{s_{0}}$. At a given $\sqrt{s}$, the amount of MPI depends on $p_{\mathrm{T} 0}$, the parton distribution functions (PDF), and the overlap of the matter distributions (or centrality) of the two colliding hadrons. Smaller values of $p_{\mathrm{T} 0}$ provide more MPI due to a larger MPI cross section. Table 1 shows the parameters in PYTHIA6 [1] and PYTHIA8 [5] that, together with the selected PDF, determine the energy dependence of MPI. Recently, in HERWIG++ $[6,7]$ the same formula has been adopted to provide an energy dependence to their MPI cutoff, which is also shown in Table 1. The QCD MC generators have other parameters that can be adjusted to control the modelling of the properties of the events, and a specified set of such parameters adjusted to fit certain prescribed aspects of the data is referred to as a "tune" [8-10].

In addition to hard-scattering processes, other processes contribute to the inelastic cross section in hadron-hadron collisions: single-diffraction dissociation (SD), doublediffraction dissociation (DD), and central-diffraction (CD). 
Table 1 Parameters in PYTHIA6 [1], PYTHIA8 [5], and HERWIG++ [6,7] MC event generators that, together with some chosen PDF, determine the energy dependence of MPI

\begin{tabular}{llll}
\hline Parameter & PYTHIA6 & PYTHIA8 & HERWIG++ \\
\hline MPI cutoff, $p_{\mathrm{T} 0}^{\text {ref }}$ at $\sqrt{s}=\sqrt{s_{0}}$ & PARP(82) & MultipartonInteractions:pT0Ref & MPIHandler:pTmin0 \\
Reference energy, $\sqrt{s_{0}}$ & PARP(89) & MultipartonInteractions:ecmRef & MPIHandler:ReferenceScale \\
Exponent of $\sqrt{s}$ dependence, $\epsilon$ & PARP(90) & MultipartonInteractions:ecmPow & MPIHandler:Power \\
\hline
\end{tabular}

In SD and DD events, one or both beam particles are excited into high-mass color-singlet states (i.e. into some resonant $\mathrm{N}^{*}$ ), which then decay. The SD and DD processes correspond to color-singlet exchanges between the beam hadrons, while CD corresponds to double color-singlet exchange with a diffractive system produced centrally. For non-diffractive processes (ND), color is exchanged, the outgoing remnants are no longer color singlets, and this separation of color generates a multitude of quark-antiquark pairs that are created via vacuum polarization. The sum of all components except SD corresponds to non single-diffraction (NSD) processes.

Minimum bias (MB) is a generic term that refers to events selected by requiring minimal activity within the detector. This selection accepts a large fraction of the overall inelastic cross section. Studies of the UE are often based on MB data, but it should be noted that the dominant particle production mechanisms in MB collisions and in the UE are not exactly the same. On the one hand, the UE is studied in collisions in which a hard 2-to-2 parton-parton scattering has occurred, by analyzing the hadronic activity in different regions of the event relative to the back-to-back azimuthal structure of the hardest particles emitted [11]. On the other hand, MB collisions are often softer and include diffractive interactions that, in the case of PYTHIA, are modelled via a Regge-based approach [12].

The MPI are usually much softer than primary hard scatters, however, occasionally two hard 2-to-2 parton scatters can take place within the same hadron-hadron collision. This is referred to as double-parton scattering (DPS) [13-16], and is typically described in terms of an effective cross section parameter, $\sigma_{\text {eff }}$, defined as:

$\sigma_{\mathrm{AB}}=\frac{\sigma_{\mathrm{A}} \sigma_{\mathrm{B}}}{\sigma_{\mathrm{eff}}}$,

where $\sigma_{\mathrm{A}}$ and $\sigma_{\mathrm{B}}$ are the inclusive cross sections for individual hard scattering processes of generic type $\mathrm{A}$ and $\mathrm{B}$, respectively, and $\sigma_{\mathrm{AB}}$ is the cross section for producing both scatters in the same hadron-hadron collision. If A and B are indistinguishable, as in four-jet production, a statistical factor of $1 / 2$ must be inserted on the right-hand side of Eq. (3). Furthermore, $\sigma_{\text {eff }}$ is assumed to be independent of $\mathrm{A}$ and B. However, $\sigma_{\text {eff }}$ is not a directly observed quantity, but can be calculated from the overlap function of the two transverse profile distributions of the colliding hadrons, as implemented in any given MPI model.

The UE tunes have impact in both soft and hard particle production in a given pp collision. First, about half of the particles produced in a MB collision originate from the hadronization of partons scattered in MPI, and have their differential cross sections in $p_{\mathrm{T}}$ regulated via Eq. (1), using the same $p_{\mathrm{T} 0}$ cutoff used to tame the hardest 2-to-2 parton-parton scattering in the event. The tuning of the cross-section regularization affects therefore all (soft and hard) parton-parton scatterings and provides a prediction for the behavior of the ND cross section. Second, the UE tunes parametrize the distribution in the transverse overlap of the colliding protons and thereby the probability of two hard parton-parton scatters that is then used to estimate DPS-sensitive observables.

In this paper, we study the $\sqrt{s}$ dependence of the UE using recent CDF proton-antiproton data from the Fermilab Tevatron at $0.3,0.9$, and $1.96 \mathrm{TeV}$ [11], together with CMS pp data from the CERN LHC at $\sqrt{s}=7 \mathrm{TeV}$ [17]. The 0.3 and $0.9 \mathrm{TeV}$ data are from the "Tevatron energy scan" performed just before the Tevatron was shut down. Using the RIVET (version 1.9.0) and PROFESSOR (version 1.3.3) frameworks [18,19], we construct: (i) new PYTHIA8 (version 8.185) UE tunes using several PDF sets (CTEQ6L1 [20], HERAPDF1.5LO [21], and NNPDF2.3LO [22,23]), (ii) new PYTHIA6 (version 6.327) UE tunes (using CTEQ6L1 and HERAPDF1.5LO), and (iii) a new HERWIG++ (version 2.7.0) UE tune for CTEQ6L1. The RIVET software is a tool for producing predictions of physics quantities obtained from MC event generators. It is used for generating sets of MC predictions with a different choice of parameters related to the UE simulation. The predictions are then included in the PROFESSOR framework, which parametrizes the generator response and returns the set of tuned parameters that best fits the input measurements.

In addition, we construct several new CMS "DPS tunes" and investigate whether the values of the UE parameters determined from fitting the UE observables in a hardscattering process are consistent with the values determined from fitting DPS-sensitive observables. The PROFESSOR software also offers the possibility of extracting "eigentunes", which provide an estimate of the uncertainties in the fitted parameters. The eigentunes consist of a collection of additional tunes, obtained through the covariance matrix of 
the data-theory fitting procedure, to determine independent directions in parameter space that provide a specific modification in the goodness of the fit, $\chi^{2}$ (Sect. 2). All of the CMS UE and DPS tunes are provided with eigentunes. In Sect. 4, predictions using the CMS UE tunes are compared to other UE measurements not used in determining the tunes, and we examine how well Drell-Yan, MB, and multijet observables can be predicted using the UE tunes. In Sect. 5, predictions of the new tunes are shown for UE observables at $13 \mathrm{TeV}$, together with a comparison to the first MB distribution measured. Section 6 has a brief summary and conclusions. The appendices contain additional comparisons between the PYTHIA6 and HERWIG++ UE tunes and the data, information about the tune uncertainties, and predictions for some MB and DPS observables at $13 \mathrm{TeV}$.

\section{The CMS UE tunes}

Previous UE studies have used the charged-particle jet with largest $p_{\mathrm{T}}[24,25]$ or a $\mathrm{Z}$ boson $[11,26]$ as the leading (i.e. highest $p_{\mathrm{T}}$ ) objects in the event. The CDF and CMS data, used for the tunes, select the charged particle with largest $p_{\mathrm{T}}$ in the event $\left(p_{\mathrm{T}}^{\max }\right)$ as the "leading object", and use just the charged particles with $p_{\mathrm{T}}>0.5 \mathrm{GeV}$ and $|\eta|<0.8$ to characterize the UE.

On an event-by-event basis, the leading object is used to define regions of pseudorapidity-azimuth $(\eta-\phi)$ space. The "toward" region relative to this direction, as indicated in Fig. 1, is defined by $|\Delta \phi|<\pi / 3$ and $|\eta|<0.8$, and the "away" region by $|\Delta \phi|>2 \pi / 3$ and $|\eta|<0.8$. The chargedparticle and the scalar- $p_{\mathrm{T}}$ sum densities in the transverse region are calculated as the sum of the contribution in the two regions: "Transverse-1" $(\pi / 3<\Delta \phi<2 \pi / 3,|\eta|<0.8)$ and
"Transverse-2" $(\pi / 3<-\Delta \phi<2 \pi / 3,|\eta|<0.8)$, divided by the area in $\eta-\phi$ space, $\Delta \eta \Delta \phi=1.6 \times 2 \pi / 3$. The transverse region is further separated into the "TransMAX" and "TransMIN" regions, also shown in Fig. 1. This defines on an eventby-event basis the regions with more (TransMAX) and fewer (TransMIN) charged particles $\left(\mathrm{N}_{\mathrm{ch}}\right)$, or greater (TransMAX) or smaller (TransMIN) scalar- $p_{\mathrm{T}}$ sums $\left(p_{\mathrm{T}}^{\text {sum }}\right)$. The UE particle and $p_{\mathrm{T}}$ densities are constructed by dividing by the area in $\eta-\phi$ space, where the TransMAX and TransMIN regions each have an area of $\Delta \eta \Delta \phi=1.6 \times 2 \pi / 6$. The transverse density (also referred to as "TransAVE") is the average of the TransMAX and the TransMIN densities. For events with hard initial- or final-state radiation, the TransMAX region often contains a third jet, but both the TransMAX and TransMIN regions receive contributions from the MPI and beam-beam remnant components. The TransMIN region is very sensitive to the MPI and beam-beam remnant components of the UE, while "TransDIF" (the difference between TransMAX and TransMIN densities) is very sensitive to ISR and FSR [27].

The new UE tunes are determined by fitting UE observables, and using only those parameters that are most sensitive to the UE data. Since it is not possible to tune all parameters of a MC event generator at once, the parameters that affect, for example, the parton shower, the fragmentation, and the intrinsic-parton $p_{\mathrm{T}}$ are fixed to the values given by an initially established reference tune. The initial reference tunes used for PYTHIA8 are Tune 4C [28] and the Monash Tune [29]. For PYTHIA6, the reference tune is Tune Z2*lep [25], and for HERWIG++ it is Tune UE-EE-5C [30].

\subsection{The PYTHIA8 UE tunes}

Taking as the reference tune the set of parameters of PYTHIA8 Tune 4C [28], we construct two new UE tunes,

Fig. 1 Left Illustration of the azimuthal regions in an event defined by the $\Delta \phi$ angle relative to the direction of the leading object [11]. Right Illustration of the topology of a hadron-hadron collision in which a hard parton-parton collision has occurred, and the leading object is taken to be the charged particle of largest $p_{\mathrm{T}}$ in the event, $p_{\mathrm{T}}^{\max }$
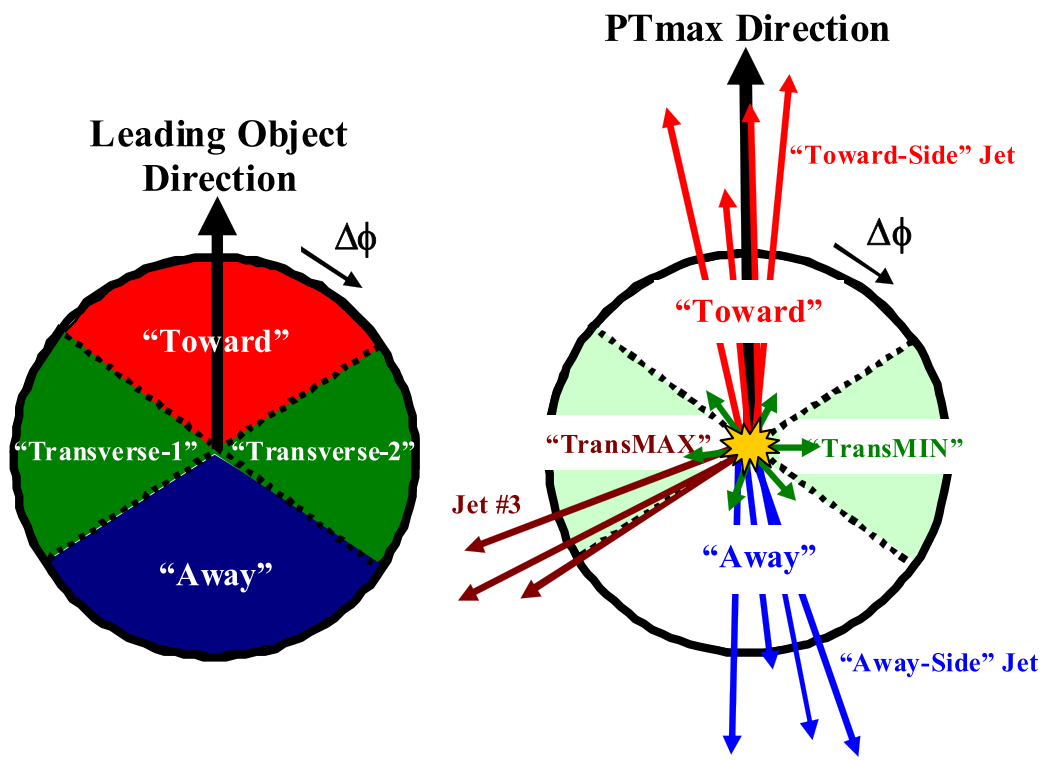
Table 2 The pythiA8 parameters, tuning range, Tune $4 \mathrm{C}$ values [28], and best-fit values for CUETP8S1-CTEQ6L1 and CUETP8S1HERAPDF1.5LO, obtained from fits to the TransMAX and TransMIN charged-particle and $p_{\mathrm{T}}^{\text {sum }}$ densities, as defined by the leading chargedparticle $p_{\mathrm{T}}^{\max }$ at $\sqrt{s}=0.9,1.96$, and $7 \mathrm{TeV}$. The $\sqrt{s}=300 \mathrm{GeV}$ data are excluded from the fit

\begin{tabular}{|c|c|c|c|c|}
\hline PYTHIA8 parameter & Tuning range & Tune $4 \mathrm{C}$ & CUETP8S1 & CUETP8S1 \\
\hline PDF & - & CTEQ6L1 & CTEQ6L1 & HERAPDF1.5LO \\
\hline MultipartonInteractions:pT0Ref [GeV] & $1.0-3.0$ & 2.085 & 2.101 & 2.000 \\
\hline MultipartonInteractions:ecmPow & $0.0-0.4$ & 0.19 & 0.211 & 0.250 \\
\hline MultipartonInteractions:expPow & $0.4-10.0$ & 2.0 & 1.609 & 1.691 \\
\hline ColourReconnection:range & $0.0-9.0$ & 1.5 & 3.313 & 6.096 \\
\hline MultipartonInteractions:ecmRef $[\mathrm{GeV}]$ & - & 1800 & $1800^{\mathrm{a}}$ & $1800^{\mathrm{a}}$ \\
\hline$\chi^{2} / \mathrm{dof}$ & - & - & 0.952 & 1.13 \\
\hline
\end{tabular}

${ }^{a}$ Fixed at Tune $4 \mathrm{C}$ value

one using CTEQ6L1 (CUETP8S1-CTEQ6L1) and one using HERAPDF1.5LO (CUETP8S1-HERAPDF1.5LO). CUET (read as "cute") stands for "CMS UE tune", and P8S1 stands for PYTHIA8 "Set 1".

The tunes are extracted by varying the four parameters in Table 2 in fits to the TransMAX and TransMIN chargedparticle and $p_{\mathrm{T}}^{\text {sum }}$ densities at three energies, for $\mathrm{p} \overline{\mathrm{p}}$ collisions at $\sqrt{s}=0.9$ and 1.96 , and pp collisions at $7 \mathrm{TeV}$. The measurements of TransAVE and TransDIF densities are not included in the fit, since they can be constructed from TransMAX and TransMIN. The new tunes use an exponentiallyfalling matter-overlap function between the two colliding protons of the form $\exp \left(-b^{\text {expPow }}\right)$, with $b$ being the impact parameter of the collision. The parameters that are varied are expPow, the MPI energy-dependence parameters (Table 1) and the range, i.e. the probability, of color reconnection (CR). A small (large) value of the final-state CR parameter tends to increase (reduce) the final particle multiplicities. In PYTHIA8, unlike in PYTHIA6, only one parameter determines the amount of $\mathrm{CR}$, which includes a $p_{\mathrm{T}}$ dependence, as defined in Ref. [5].

The generated inelastic events include ND and diffractive (DD+SD+CD) contributions, although the UE observables used to determine the tunes are sensitive to single-diffraction dissociation, central-diffraction, and double-diffraction dissociation only at very small $p_{\mathrm{T}}^{\max }$ values (e.g. $p_{\mathrm{T}}^{\max }<$ $1.5 \mathrm{GeV})$. The ND component dominates for $p_{\mathrm{T}}^{\max }$ values greater than $\approx 2.0 \mathrm{GeV}$, since the cross section of the diffractive components rapidly decreases as a function of $\hat{p}_{\mathrm{T}}$. The fit is performed by minimizing the $\chi^{2}$ function:

$\chi^{2}(p)=\sum_{i} \frac{\left(f^{i}(p)-R_{i}\right)^{2}}{\Delta_{i}^{2}}$,

where the sum runs over each bin $i$ of every observable. The $f^{i}(p)$ functions correspond to the interpolated MC response for the simulated observables as a function of the parameter vector $p, R_{i}$ is the value of the measured observable in bin $i$, and $\Delta_{i}$ is the total experimental uncertainty of $R_{i}$. We do not use the Tevatron data at $\sqrt{s}=300 \mathrm{GeV}$, as we are unable to obtain an acceptable $\chi^{2}$ in a fit of the four parameters in Table 2. The $\chi^{2}$ per degree of freedom (dof) listed in Table 2 refers to the quantity $\chi^{2}(p)$ in Eq. (4), divided by the number of dof in the fit. The eigentunes (Appendix A) correspond to the tunes in which the changes in the $\chi^{2}\left(\Delta \chi^{2}\right)$ of the fit relative to the best-fit value equals the $\chi^{2}$ value obtained in the tune, i.e. $\Delta \chi^{2}=\chi^{2}$. For both tunes in Table 2, the fit quality is very good, with $\chi^{2} /$ dof values very close to 1 .

The contribution from CR changes in the two new tunes; it is large for the HERAPDF1.5LO and small for the CTEQ6L1 PDF. This is a result of the shape of the parton densities at small fractional momenta $x$, which is different for the two PDF sets. While the parameter $p_{\mathrm{T} 0}^{\text {ref }}$ in Eq. (2) stays relatively constant between Tune 4C and the new tunes, the energy dependence $\epsilon$ tends to increase in the new tunes, as do the matter-overlap profile functions.

The PYTHIA8 Monash Tune [29] combines updated fragmentation parameters with the NNPDF2.3LO PDF.

The NNPDF2.3LO PDF has a gluon distribution at small $x$ that is different compared to CTEQ6L1 and HERAPDF1.5LO, and this affects predictions in the forward region of hadron-hadron collisions. Tunes using the NNPDF2.3LO PDF provide a more consistent description of the UE and MB observables in both the central and forward regions, than tunes using other PDF.

A new PYTHIA8 tune CUETP8M1 (labeled with $\mathrm{M}$ for Monash) is constructed using the parameters of the Monash Tune and fitting the two MPI energy-dependence parameters of Table 1 to UE data at $\sqrt{s}=0.9,1.96$, and $7 \mathrm{TeV}$. Varying the $\mathrm{CR}$ range and the exponential slope of the matter-overlap function freely in the minimization of the $\chi^{2}$ leads to suboptimal best-fit values. The CR range is therefore fixed to the value of the Monash Tune, and the exponential slope of the matter-overlap function expPow is set to 1.6, which is similar to the value determined in CUETP8S1-CTEQ6L1. 
Table 3 The PYTHIA8 parameters, tuning range, Monash values [29], and best-fit values for CUETP8M1, obtained from fits to the TransMAX and TransMIN charged-particle and $p_{\mathrm{T}}^{\text {sum }}$ densities, as defined by the leading charged-particle $p_{\mathrm{T}}^{\max }$ at $\sqrt{s}=0.9,1.96$, and $7 \mathrm{TeV}$. The $\sqrt{s}=300 \mathrm{GeV}$ data are excluded from the fit

\begin{tabular}{llll}
\hline PYTHIA8 parameter & Tuning range & Monash & CUETP8M1 \\
\hline PDF & - & NNPDF2.3LO & NNPDF2.3LO \\
MultipartonInteractions:pT0Ref [GeV] & $1.0-3.0$ & 2.280 & 2.402 \\
MultipartonInteractions:ecmPow & $0.0-0.4$ & 0.215 & 0.252 \\
MultipartonInteractions:expPow & - & 1.85 & $1.6^{\mathrm{a}}$ \\
ColourReconnection:range & - & 1.80 & $1.80^{\mathrm{b}}$ \\
MultipartonInteractions:ecmRef [GeV] & - & 7000 & $7000^{\mathrm{b}}$ \\
$\chi^{2} /$ dof & - & - & 1.54 \\
\hline
\end{tabular}

${ }^{a}$ Fixed at CUETP8S1-CTEQ6L1 value

${ }^{\mathrm{b}}$ Fixed at Monash Tune value

TransMIN charged-particle density $\sqrt{s}=300 \mathrm{GeV}$

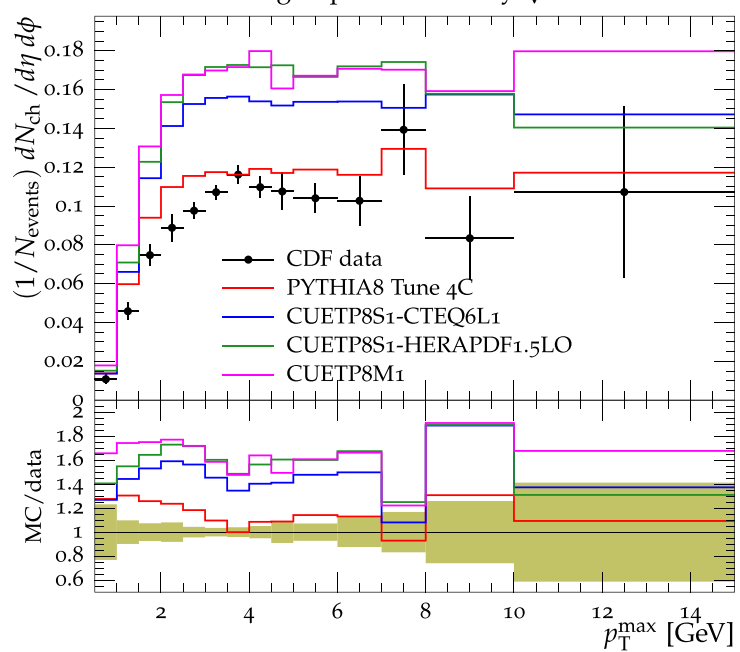

TransMIN charged $p_{\mathrm{T}}^{\text {sum }}$ density $\sqrt{s}=300 \mathrm{GeV}$

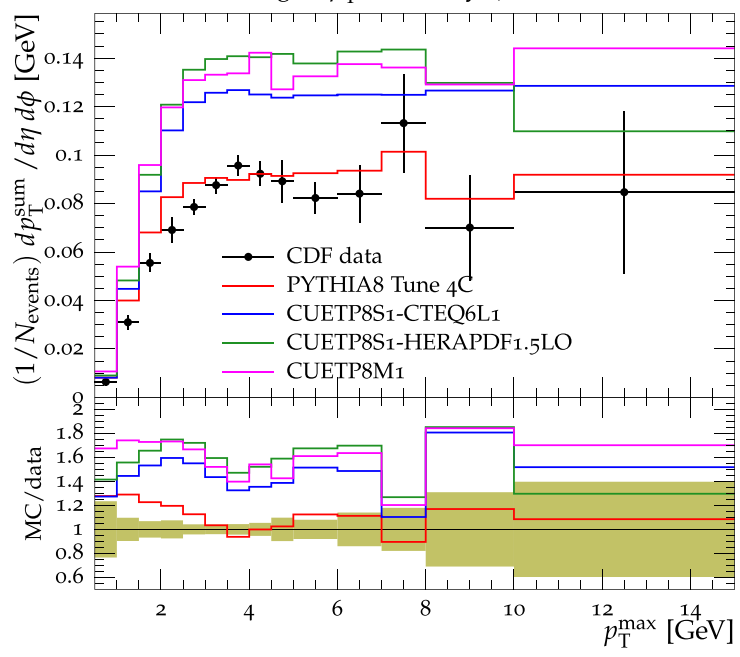

Fig. $2 \mathrm{CDF}$ data at $\sqrt{s}=300 \mathrm{GeV}[11]$ on particle (top) and $p_{\mathrm{T}}^{\text {sum }}$ densities (bottom) for charged particles with $p_{\mathrm{T}}>0.5 \mathrm{GeV}$ and $|\eta|<0.8$ in the TransMIN (left) and TransMAX (right) regions as defined by the leading charged particle, as a function of the transverse momentum of the leading charged-particle $p_{\mathrm{T}}^{\max }$. The data are compared to PYTHIA8
TransMAX charged-particle density $\sqrt{s}=300 \mathrm{GeV}$

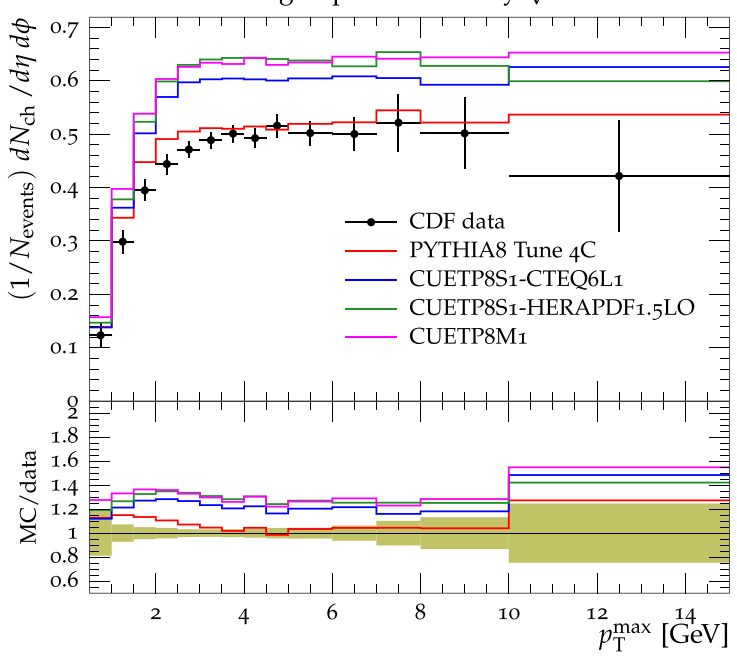

TransMAX charged $p_{\mathrm{T}}^{\text {sum }}$ density $\sqrt{s}=300 \mathrm{GeV}$

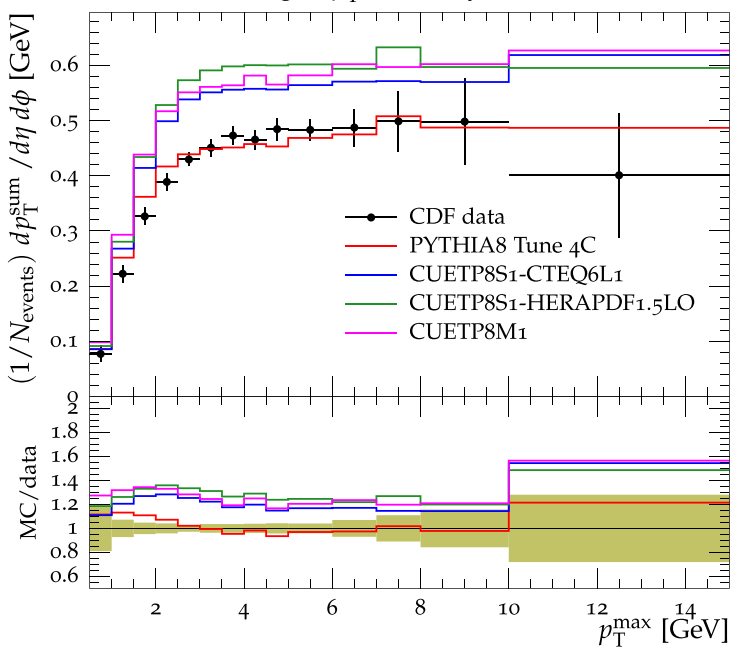

Tune 4C, CUETP8S1-CTEQ6L1, CUETP8S1-HERAPDF1.5LO, and CUETP8M1. The ratios of MC events to data are given below each panel. The data at $\sqrt{s}=300 \mathrm{GeV}$ are not used in determining these tunes. The green bands in the ratios represent the total experimental uncertainties 


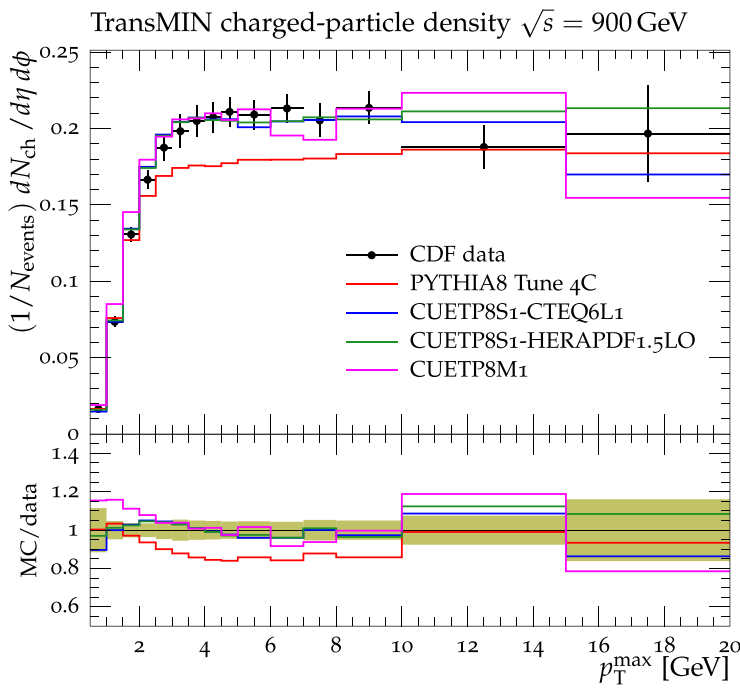

TransMIN charged $p_{\mathrm{T}}^{\text {sum }}$ density $\sqrt{s}=900 \mathrm{GeV}$

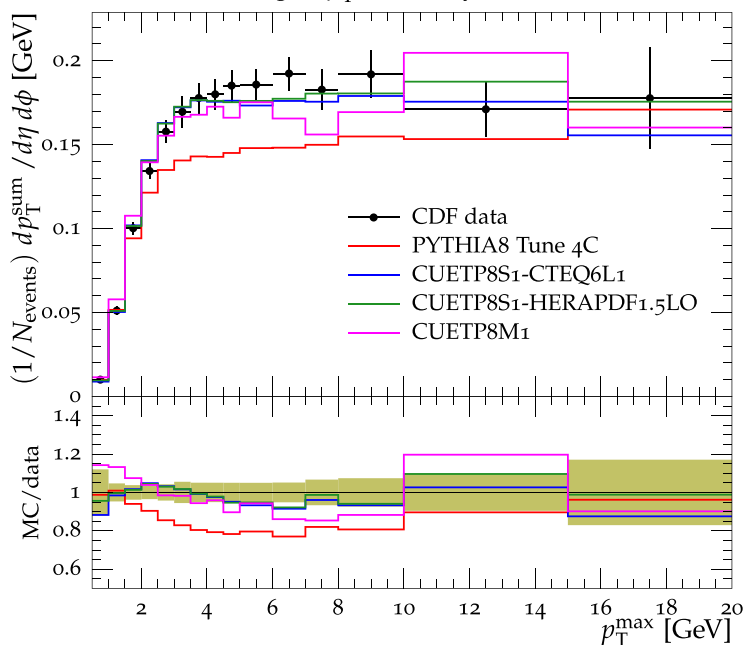

Fig. $3 \mathrm{CDF}$ data at $\sqrt{s}=900 \mathrm{GeV}[11]$ on particle (top) and $p_{\mathrm{T}}^{\text {sum }}$ densities (bottom) for charged particles with $p_{\mathrm{T}}>0.5 \mathrm{GeV}$ and $|\eta|<0.8$ in the TransMIN (left) and TransMAX (right) regions as defined by the leading charged particle, as a function of the transverse momentum of the leading charged-particle $p_{\mathrm{T}}^{\max }$. The data are compared to PYTHIA8

The best-fit values of the two tuned parameters are shown in Table 3. Again, we exclude the $300 \mathrm{GeV}$ data, since we are unable to get a good $\chi^{2}$ in the fit. The parameters obtained for CUETP8M1 differ slightly from the ones of the Monash Tune. The obtained energy-dependence parameter $\epsilon$ is larger, while a very similar value is obtained for $p_{\mathrm{T} 0}^{\text {ref }}$.

Figures 2, 3, 4 and 5 show the CDF data at 0.3, 0.9, and $1.96 \mathrm{TeV}$, and the CMS data at $7 \mathrm{TeV}$ for charged-particle and $p_{\mathrm{T}}^{\text {sum }}$ densities in the TransMIN and TransMAX regions as a function of $p_{\mathrm{T}}^{\max }$, compared to predictions obtained with the PYTHIA8 Tune $4 \mathrm{C}$ and with the new CMS tunes: CUETP8S1-CTEQ6L1, CUETP8S1-HERAPDF1.5LO, and CUETP8M1. Predictions from the new tunes cannot reproduce the $\sqrt{s}=300 \mathrm{GeV}$ data, but describe very well the data
TransMAX charged-particle density $\sqrt{s}=900 \mathrm{GeV}$

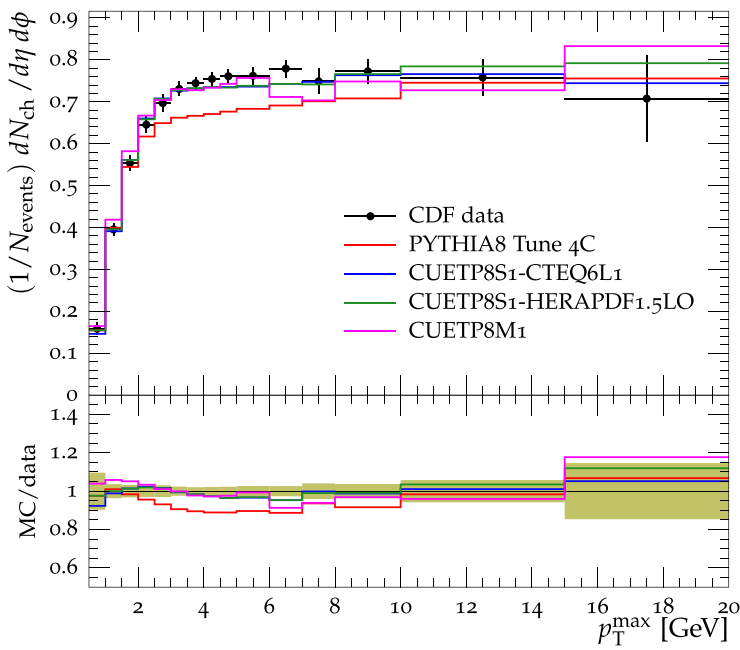

TransMAX charged $p_{\mathrm{T}}^{\text {sum }}$ density $\sqrt{s}=900 \mathrm{GeV}$

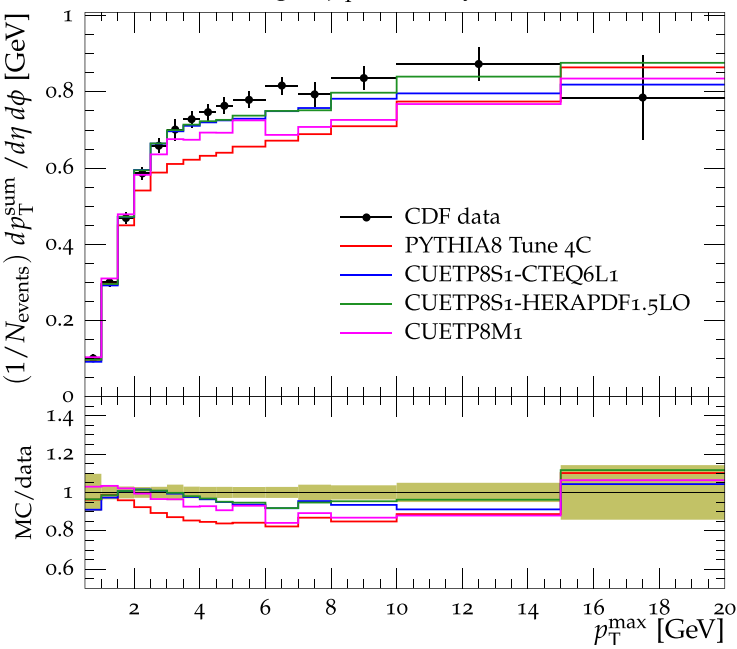

Tune 4C, CUETP8S1-CTEQ6L1, CUETP8S1-HERAPDF1.5LO, and CUETP8M1. The ratios of MC events to data are given below each panel. The green bands in the ratios represent the total experimental uncertainties

at the higher $\sqrt{s}=0.9,1.96$, and $7 \mathrm{TeV}$. In particular, the description provided by the new tunes significantly improves relative to the old Tune $4 \mathrm{C}$, which is likely due to the better choice of parameters used in the MPI energy dependence and the extraction of the $\mathrm{CR}$ in the retuning.

\subsection{The PYTHIA6 UE tunes}

The PYTHIA6 Tune Z2*lep [25] uses the improved fragmentation parameters from fits to the $\mathrm{LEP} \mathrm{e}^{+} \mathrm{e}^{-}$data [31], and a double-Gaussian matter profile for the colliding protons but corresponds to an outdated CMS UE tune. It was constructed by fitting the CMS charged-particle jet UE data at 0.9 and $7 \mathrm{TeV}$ [24] using data on the TransAVE charged-particle and 

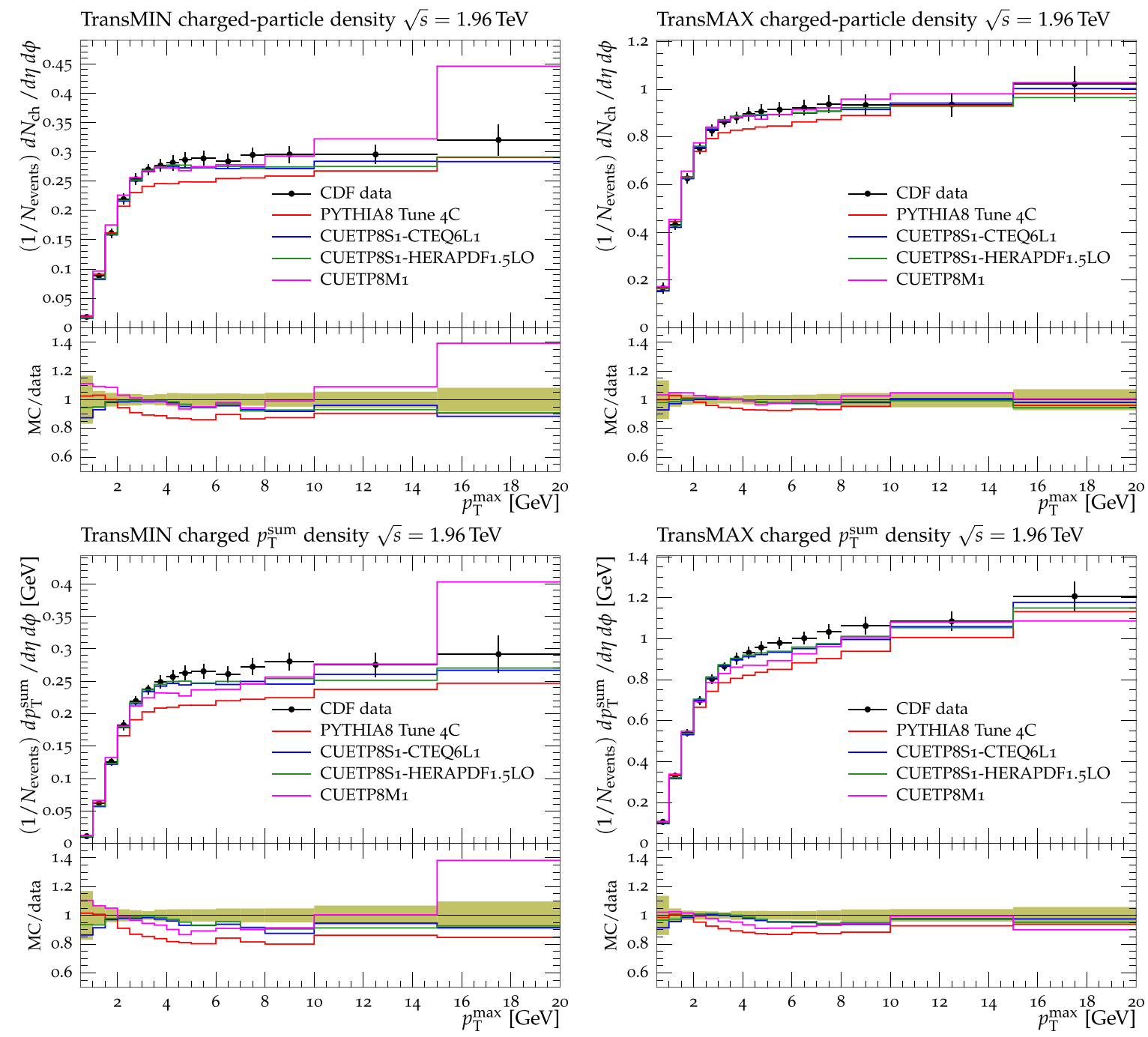

Fig. $4 \mathrm{CDF}$ data at $\sqrt{s}=1.96 \mathrm{TeV}[11]$ on particle (top) and $p_{\mathrm{T}}^{\text {sum }}$ densities (bottom) for charged particles with $p_{\mathrm{T}}>0.5 \mathrm{GeV}$ and $|\eta|<0.8$ in the TransMIN (left) and TransMAX (right) regions as defined by the leading charged particle, as a function of the transverse momentum of the leading charged-particle $p_{\mathrm{T}}^{\max }$. The data are compared to PYTHIA8

Tune 4C, CUETP8S1-CTEQ6L1, CUETP8S1-HERAPDF1.5LO, and CUETP8M1. The ratios of MC events to data are given below each panel. The green bands in the ratios represent the total experimental uncertainties

$p_{\mathrm{T}}^{\text {sum }}$ densities, since data on TransMAX, TransMIN, and TransDIF were not available at that time.

Starting with Tune Z2*lep parameters, two new PYTHIA6 UE tunes are constructed, one using CTEQ6L1 (CUETP6S1CTEQ6L1) and one using HERAPDF1.5LO (CUETP6S1HERAPDF1.5LO), with P6S1 standing for PYTHIA6 "Set 1". The tunes are constructed by fitting the five parameters shown in Table 4 to the TransMAX and TransMIN charged-particle and $p_{\mathrm{T}}^{\text {sum }}$ densities at $\sqrt{s}=0.3,0.9,1.96$, and $7 \mathrm{TeV}$. In addition to varying the MPI energy-dependence parameters (Table 1), we also vary the core-matter fraction $\operatorname{PARP}(83)$, which parametrizes the amount of matter contained within the radius of the proton core, the CR strength PARP(78), and the CR suppression PARP(77). The PARP(78) parameter

reflects the probability for a given string to retain its color history, and therefore does not change the color and other string pieces, while the PARP(77) parameter introduces a $p_{\mathrm{T}}$ dependence on the CR probability [1].

Inelastic events $(\mathrm{ND}+\mathrm{DD}+\mathrm{SD}+\mathrm{CD})$ are generated with PYTHIA6. The best-fit values of the five parameters are shown in Table 4. The matter-core fraction is quite different in the two new PYTHIA6 tunes. This is due to the fact that this parameter is very sensitive to the behaviour of the PDF at small $x$. Predictions obtained with PYTHIA6 Tune Z2*lep , CUETP6S1-CTEQ6L1 and CUETP6S1-HERAPDF1.5LO are compared in Appendix B to the UE data. The new PYTHIA6 tunes significantly improve the description of the UE data relative to PYTHIA6 Tune $\mathrm{Z2}{ }^{*}$ lep at all considered 

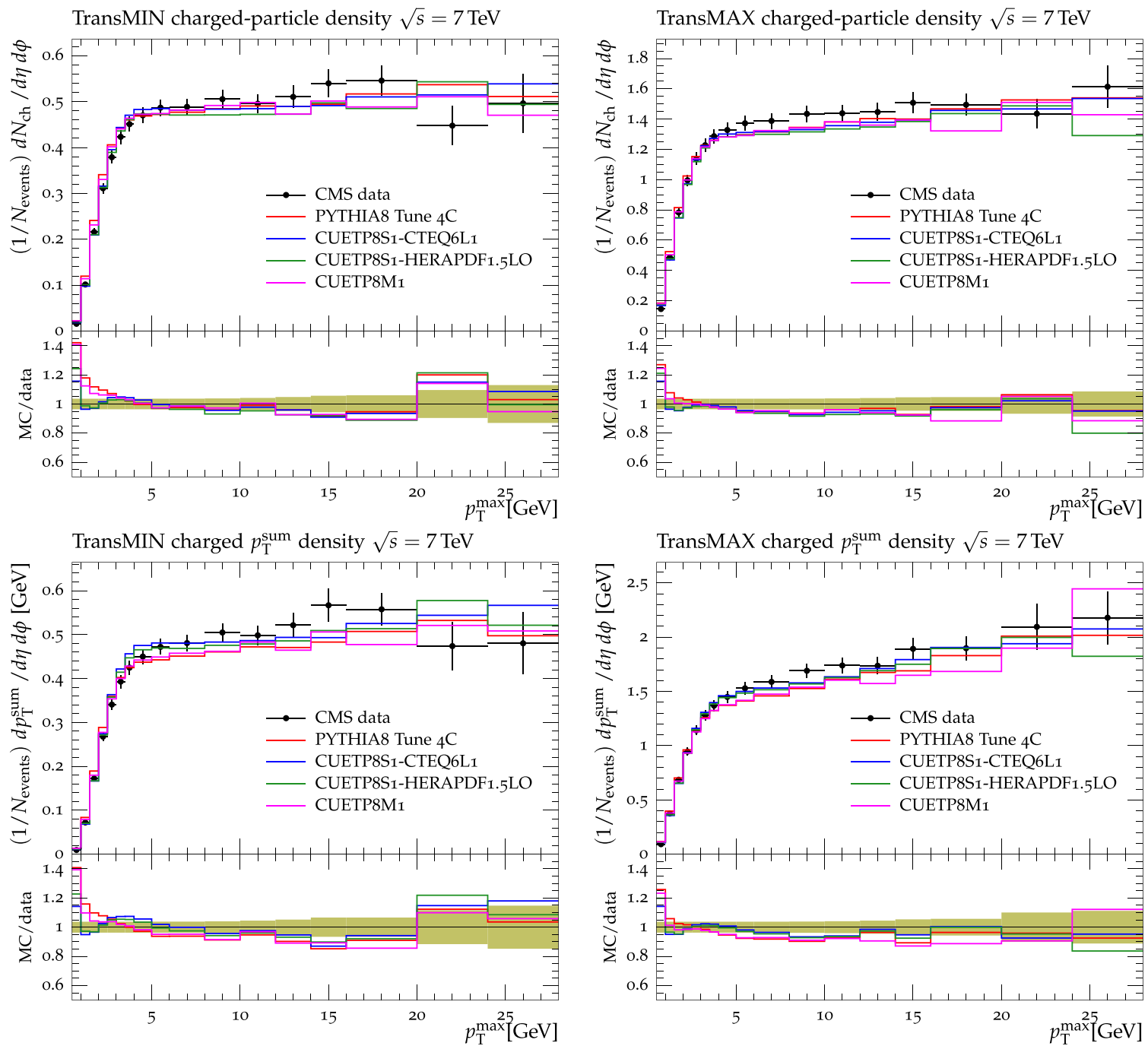

Fig. 5 CMS data at $\sqrt{s}=7 \mathrm{TeV}$ [17] on particle (top) and $p_{\mathrm{T}}^{\text {sum }}$ densities (bottom) for charged particles with $p_{\mathrm{T}}>0.5 \mathrm{GeV}$ and $|\eta|<0.8$ in the TransMIN (left) and TransMAX (right) regions as defined by the leading charged particle, as a function of the transverse momentum of the leading charged-particle $p_{\mathrm{T}}^{\max }$. The data are compared to PYTHIA8

Tune 4C, and CUETP8S1-CTEQ6L1, CUETP8S1-HERAPDF1.5LO, and CUETP8M1. The ratios of MC events to data are given below each panel. The green bands in the ratios represent the total experimental uncertainties

energies, due to the better choice of parameters governing the MPI energy dependence.

\subsection{The HERWIG++ UE tunes}

Starting with the parameters of HERWIG++ Tune UEEE-5C [30], we construct a new HERWIG++ UE tune, CUETHppS1, where Hpp stands for HERWIG++. This tune is obtained by varying the four parameters shown in Table 5 in the fit to TransMAX and TransMIN charged-particle and $p_{\mathrm{T}}^{\text {sum }}$ densities at the four $\sqrt{s}=0.3,0.9,1.96$, and $7 \mathrm{TeV}$. We set the MPI cutoff $p_{\mathrm{T} 0}$ and the reference energy $\sqrt{s_{0}}$ to the Tune UE-EE-5C values, and vary the MPI c.m. energy extrapolation parameter in Table 1 . We also vary

the inverse radius that determines the matter overlap and the range of CR. The CR model in HERWIG++ is defined by two parameters, one (colourDisrupt) ruling the color structure of soft interactions $\left(p_{\mathrm{T}}<p_{\mathrm{T} 0}\right)$, and one (ReconnectionProbability) giving the probability of CR without a $p_{\mathrm{T}}$ dependence for color strings. We include all four center-of-mass energies, although at each energy we exclude the first two $p_{\mathrm{T}}^{\max }$ bins. These first bins, e.g. for $p_{\mathrm{T}}^{\max }<1.5 \mathrm{GeV}$, are sensitive to single-diffraction dissociation, central-diffraction, and double-diffraction dissociation, but HERWIG++ contains only the ND component.

In Table 5, the parameters of the new CUETHppS1 are listed and compared to those from Tune UE-EE-5C. The parameters of the two tunes are very similar. The $\chi^{2} /$ dof, also 
Table 4 The PYTHIA6 parameters, tuning range, Tune Z2*lep values [31], and best-fit values for CUETP6S1-CTEQ6L1 and CUETP6S1HERAPDF1.5LO, obtained from fits to the TransMAX and TransMIN charged-particle and $p_{\mathrm{T}}^{\text {sum }}$ densities as defined by the $p_{\mathrm{T}}^{\max }$ of the leading charged particle at $\sqrt{s}=0.3,0.9,1.96$, and $7 \mathrm{TeV}$

\begin{tabular}{lllll}
\hline PYTHIA6 parameter & Tuning range & Tune Z2*lep & CUETP6S1 & CUETP6S1 \\
\hline PDF & - & CTEQ6L1 & CTEQ6L1 & HERAPDF1.5LO \\
PARP(82)-MPI cutoff $[\mathrm{GeV}]$ & $1.6-2.2$ & 1.921 & 1.910 & 0.248 \\
PARP(90)-exponent of $\sqrt{s}$ dependence & $0.18-0.28$ & 0.227 & 1.046 & 0.250 \\
PARP(77)-CR suppression & $0.25-1.15$ & 0.538 & 0.665 & 0.667 \\
PARP(78)-CR strength & $0.2-0.8$ & 0.356 & 0.822 & 0.537 \\
PARP(83)-matter fraction in core & $0.1-1.0$ & 1800 & $1800^{\mathrm{a}}$ & 0.490 \\
PARP(89)-reference energy $[\mathrm{GeV}]$ & - & - & 0.915 & $1800^{\mathrm{a}}$ \\
$\chi^{2} /$ dof & - & & 1.004 \\
\hline
\end{tabular}

${ }^{\text {a }}$ Fixed at Tune $\mathrm{Z} 2 *$ lep value

Table 5 The HERWIG++ parameters, tuning range, Tune UE-EE-5C values [30], and best-fit values for CUETHppS1, obtained from a fit to the TransMAX and TransMIN charged-particle and $p_{\mathrm{T}}^{\text {sum }}$ densities as a function of the leading charged-particle $p_{\mathrm{T}}^{\max }$ at $\sqrt{s}=0.3,0.9,1.96$, and $7 \mathrm{TeV}$

\begin{tabular}{llll}
\hline HERWIG++ parameter & Tuning range & UE-EE-5C & CUETHppS1 \\
\hline PDF & - & CTEQ6L1 & CTEQ6L1 \\
MPIHandler:Power & $0.1-0.5$ & 0.33 & 0.371 \\
RemnantDecayer:colourDisrupt & $0.1-0.9$ & 0.8 & 0.628 \\
MPIHandler:InvRadius $\left[\mathrm{GeV}^{2}\right]$ & $0.5-2.7$ & 2.30 & 2.255 \\
ColourReconnector:ReconnectionProbability & $0.1-0.9$ & 0.49 & 0.528 \\
MPIHandler:pTmin0 [GeV] & - & 3.91 & $3.91^{\mathrm{a}}$ \\
MPIHandler:ReferenceScale [GeV] & - & 7000 & $7000^{\mathrm{a}}$ \\
$\chi^{2} /$ dof & - & - & 0.463 \\
\hline
\end{tabular}

a Fixed at Tune UE-EE-5C value

Table 6 The PYTHIA8 parameters, tuning ranges, Tune 4C values [28] and best-fit values of CDPSTP8S1-Wj and CDPSTP8S2-Wj, obtained from fits to DPS observables in W+dijet production with the MAD-

\begin{tabular}{lllll}
\hline PYTHIA8 parameter & Tuning range & Tune 4C & CDPSTP8S1-Wj & CDPSTP8S2-Wj \\
\hline PDF & & CTEQ6L1 & CTEQ6L1 & CTEQ6L1 \\
MultipartonInteractions:pT0Ref [GeV] & $1.0-3.0$ & 2.085 & $2.085^{\mathrm{a}}$ & 2.501 \\
MultipartonInteractions:ecmPow & $0.0-0.4$ & 0.19 & $0.19^{\mathrm{a}}$ & 0.179 \\
MultipartonInteractions:expPow & $0.4-10.0$ & 2.0 & 1.523 & 1.120 \\
ColourReconnection:range & $0.0-9.0$ & 1.5 & $1.5^{\mathrm{a}}$ & 2.586 \\
MultipartonInteractions:ecmRef [GeV] & - & 1800 & $1800^{\mathrm{a}}$ & $1800^{\mathrm{a}}$ \\
$\chi^{2} /$ dof & - & - & 0.118 & 0.09 \\
Predicted $\sigma_{\text {eff }}(\mathrm{in} \mathrm{mb})$ & - & 30.3 & $25.9_{-2.9}^{+2.4}$ & $25.8_{-4.2}^{+8.2}$ \\
\hline
\end{tabular}

${ }^{a}$ Fixed at Tune $4 \mathrm{C}$ value

indicated in Table 5, is found to be $\approx 0.46$, which is smaller than the value obtained for other CMS UE tunes. This is due to the fact that the first two bins as a function of $p_{\mathrm{T}}^{\max }$, which have much smaller statistical uncertainties than the higher$p_{\mathrm{T}}^{\max }$ bins, are excluded from the fit because they cannot be described by any reasonable fit-values. In Appendix C, predictions obtained with HERWIG++ Tune UE-EE-5C and
GRAPH event generator interfaced to PYTHIA8. Also shown are the predicted values of $\sigma_{\text {eff }}$ at $\sqrt{s}=7 \mathrm{TeV}$, and the uncertainties obtained from the eigentunes

CUETHppS1 are compared to the UE data. The two tunes are both able to reproduce the UE data at all energies. With the new CUETHppS1 tune, uncertainties can be estimated using the eigentunes (Appendix A).

In conclusion, both HERWIG++ tunes, as well as the new CMS PYTHIA6 UE tunes reproduce the UE data at all four $\sqrt{s}$. The PYTHIA8 UE tunes, however, do not describe well 

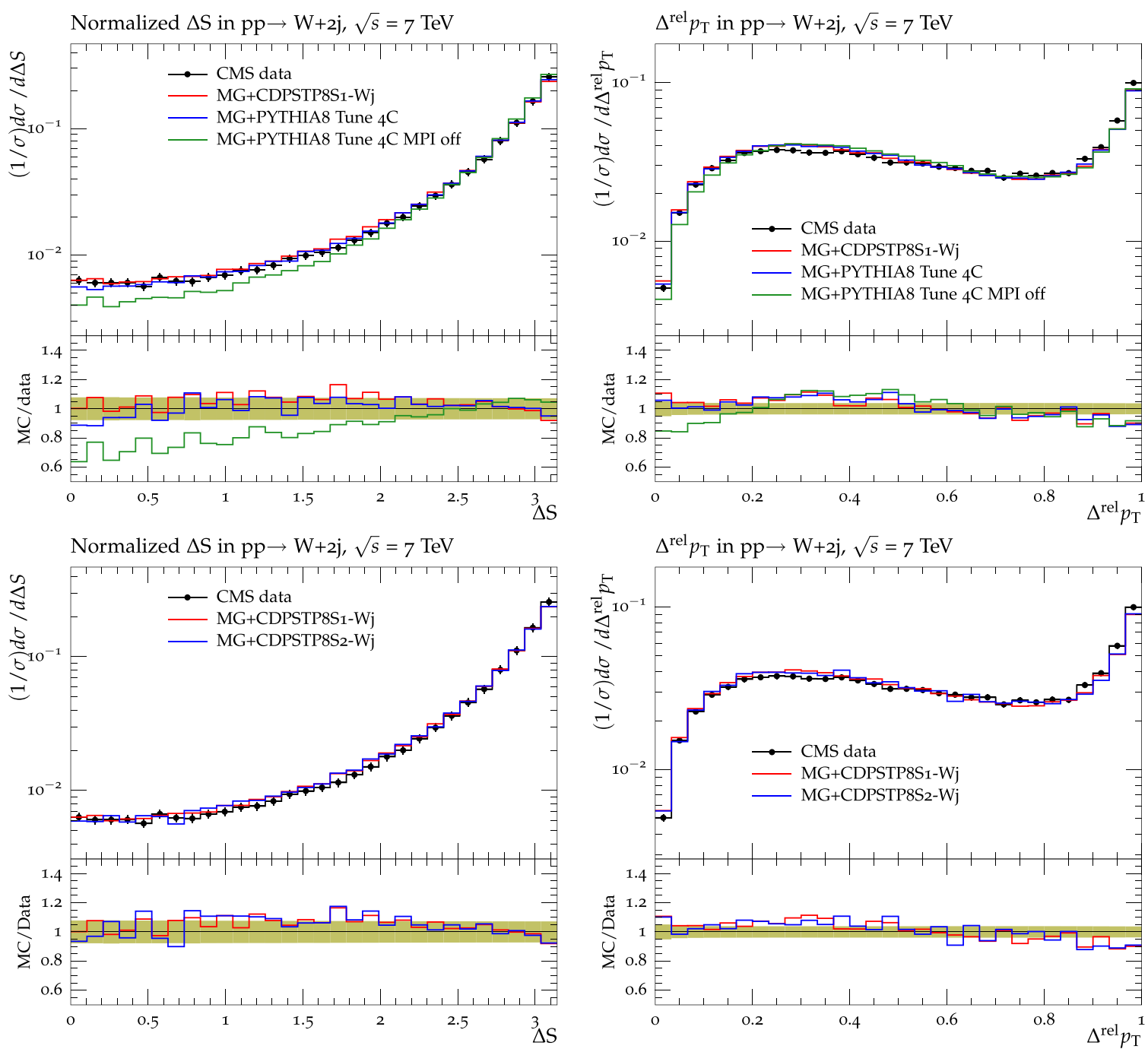

Fig. 6 CMS data at $\sqrt{s}=7 \mathrm{TeV}$ [36] for the normalized distributions of the correlation observables $\Delta \mathrm{S}$ (left), and $\Delta^{\text {rel }} p_{\mathrm{T}}$ (right) in the W+dijet channel, compared to MADGRAPH (MG) interfaced to: PYTHIA8 Tune 4C, Tune 4C with no MPI, and the CMS PYTHIA8 DPS

the data at $\sqrt{s}=300 \mathrm{GeV}$, which may be related to the modelling of the proton-proton overlap function. The PYTHIA6 Tune $\mathrm{Z2}^{*} \mathrm{lep}$, and the new CMS UE tunes use a doubleGaussian matter distribution, while all the PYTHIA8 UE tunes use a single exponential matter overlap. The HERWIG++ tune, on the other hand, uses a matter-overlap function that is related to the Fourier transform of the electromagnetic form factor with $\mu^{2}$ [7] playing the role of an effective inverse proton radius (i.e. the InvRadius parameter in Table 5). However, predictions from a tune performed with PYTHIA8 using a double-Gaussian matter distribution were not able to improve the quality of the fit as a fit obtained without interleaved FSR in the simulation of the UE (as it is implemented in PYTHIA6) did not show any improvement. Further investigations are needed to resolve this issue.

partial CDPSTP8S1-Wj (top); and CDPSTP8S1-Wj, and CDPSTP8S2$\mathrm{Wj}$ (bottom). The bottom panels of each plot show the ratios of these tunes to the data, and the green bands around unity represent the total experimental uncertainty

\section{The CMS DPS tunes}

Traditionally, $\sigma_{\text {eff }}$ is determined by fitting the DPS-sensitive observables with two templates [32-36] that are often based on distributions obtained from QCD MC models. One template is constructed with no DPS, i.e. just single parton scattering (SPS), while the other represents DPS production. This determines $\sigma_{\text {eff }}$ from the relative amounts of SPS and DPS contributions needed to fit the data. Here we use an alternative method that does not require construction of templates from MC samples. Instead, we fit the DPS-sensitive observables directly and then calculate the resulting $\sigma_{\text {eff }}$ from the model. For example, in PYTHIA8, the value of $\sigma_{\text {eff }}$ is calculated by multiplying the ND cross section by an enhancement or a depletion factor, which expresses the 
Table 7 The PYTHIA8 parameters, tuning ranges, Tune 4C values [28] and best-fit values of CDPSTP8S1-4j and CDPSTP8S2-4j, obtained from fits to DPS observables in four-jet production. Also shown are the predicted values of $\sigma_{\text {eff }}$ at $\sqrt{s}=7 \mathrm{TeV}$, and the uncertainties obtained from the eigentunes

\begin{tabular}{lllll}
\hline PYTHIA8 Parameter & Tuning range & Tune 4C & CDPSTP8S1-4j & CDPSTP8S2-4j \\
\hline PDF & & CTEQ6L1 & CTEQ6L1 & CTEQ6L1 \\
MultipartonInteractions:pT0Ref [GeV] & $1.0-3.0$ & 2.085 & $2.085^{\mathrm{a}}$ & 2.125 \\
MultipartonInteractions:ecmPow & $0.0-0.4$ & 0.19 & $0.19^{\mathrm{a}}$ & 0.179 \\
MultipartonInteractions:expPow & $0.4-10.0$ & 2.0 & 1.160 & 0.692 \\
ColourReconnection:range & $0.0-9.0$ & 1.5 & $1.5^{\mathrm{a}}$ & 6.526 \\
MultipartonInteractions:ecmRef [GeV] & - & -1800 & $1800^{\mathrm{a}}$ & $1800^{\mathrm{a}}$ \\
$\chi^{2} /$ dof & - & 30.3 & 0.751 & 0.428 \\
Predicted $\sigma_{\text {eff }}$ (in mb) & - & & $21.3_{-1.6}^{+1.2}$ & $19.0_{-3.0}^{+4.7}$ \\
\hline
\end{tabular}

${ }^{a}$ Fixed at Tune $4 \mathrm{C}$ value

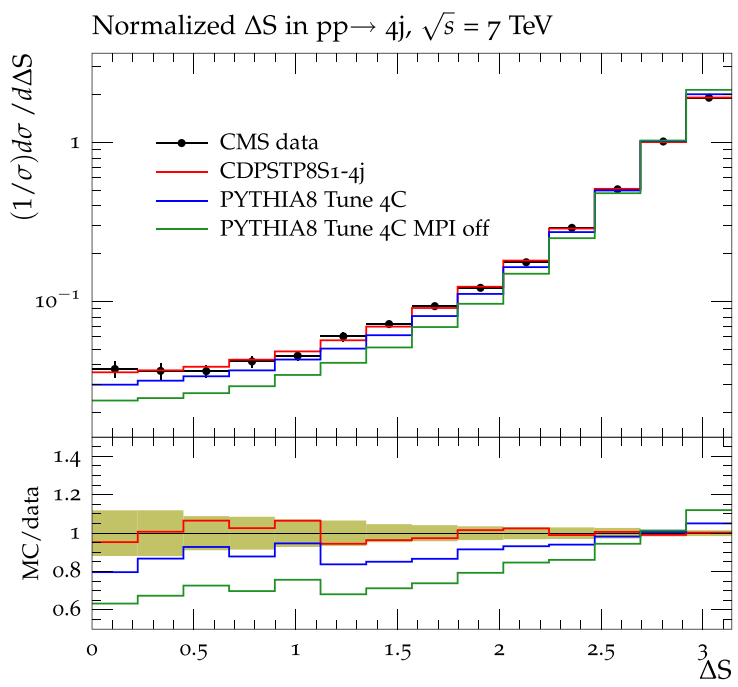

Fig. 7 Distributions of the correlation observables $\Delta \mathrm{S}$ (left) and $\Delta^{\text {rel }} p_{\mathrm{T}}($ right $)$ measured in four-jet production at $\sqrt{s}=7 \mathrm{TeV}$ [37] compared to PYTHIA8 Tune 4C, Tune 4C with no MPI, and CDPSTP8S1-4j.

dependence of DPS events on the collision impact parameter. As expected, more central collisions have a higher probability of a second hard scattering than peripheral collisions. The enhancement/depletion factors depend on the UE parameters, namely, on the parameters that characterize the matter-overlap function of the two protons, which for bProfile $=3$ is determined by the exponential parameter expPow, on the MPI regulator $p_{\mathrm{T} 0}$ in Eq. (2), and the range of the CR. PYTHIA8 Tune $4 \mathrm{C}$ gives $\sigma_{\mathrm{eff}} \approx 30.3 \mathrm{mb}$ at $\sqrt{s}=7 \mathrm{TeV}$.

In Sect. 2, we determined the MPI parameters by fitting UE data. Here we determine the MPI parameters by fitting to observables which involve correlations among produced objects in hadron-hadron collisions that are sensitive to DPS. Two such observables used in the fit, $\Delta S$ and $\Delta^{\text {rel }} p_{\mathrm{T}}$, are defined as follows:

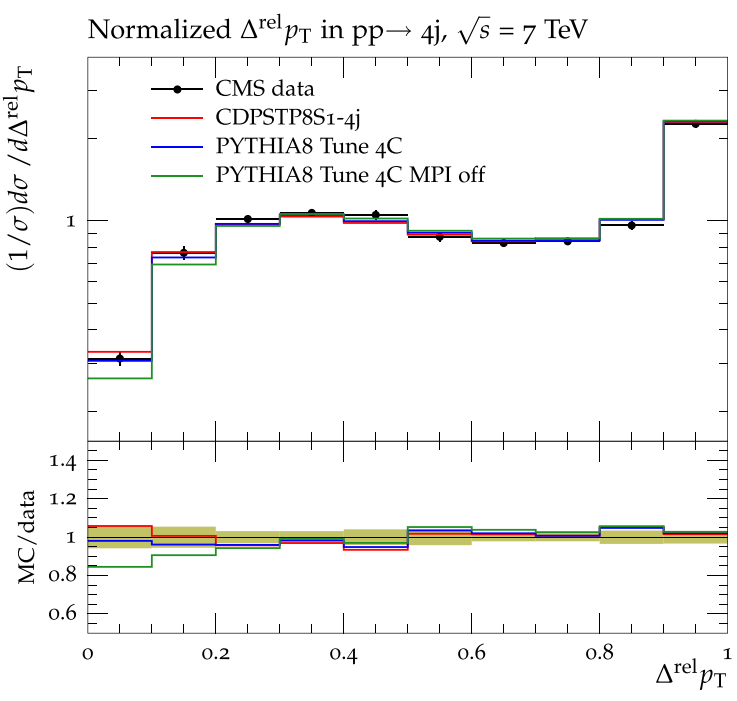

The bottom panels of each plot show the ratios of these predictions to the data, and the green bands around unity represent the total experimental uncertainty

$\Delta \mathrm{S}=\arccos \left(\frac{\vec{p}_{\mathrm{T}}\left(\text { object }_{1}\right) \cdot \vec{p}_{\mathrm{T}}\left(\text { object }_{2}\right)}{\mid \vec{p}_{T}\left(\text { object }_{1}\right)|\times| \vec{p}_{\mathrm{T}}\left(\text { object }_{2}\right) \mid}\right)$,

$\Delta^{\text {rel }} p_{\mathrm{T}}=\frac{\left|\vec{p}_{\mathrm{T}}^{\text {jet }_{1}}+\vec{p}_{\mathrm{T}}^{\text {jet }_{2}}\right|}{\left|\vec{p}_{\mathrm{T}}^{\text {jet }_{1}}\right|+\left|\vec{p}_{\mathrm{T}}^{\text {jet }_{2}}\right|}$,

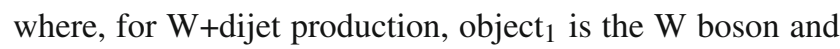
object $_{2}$ is the dijet system. For four-jet production, object ${ }_{1}$ is the hard-jet pair and object 2 is the soft-jet pair. For $\Delta^{\text {rel }} p_{\mathrm{T}}$ in $\mathrm{W}+{\text { dijet production, } \text { jet }_{1} \text { and jet }}_{2}$ are the two jets of the dijet system, while in four-jet production, jet ${ }_{1}$ and jet 2 refer to the two softer jets.

The PYTHIA8 UE parameters are fitted to the DPS-sensitive observables measured by CMS in W+dijet [36] and in fourjet production [37]. After extracting the MPI parameters, the value of $\sigma_{\text {eff }}$ in Eq. (3) can be calculated from the underlying MPI model. In PYTHIA $8, \sigma_{\text {eff }}$ depends primarily on the matter- 


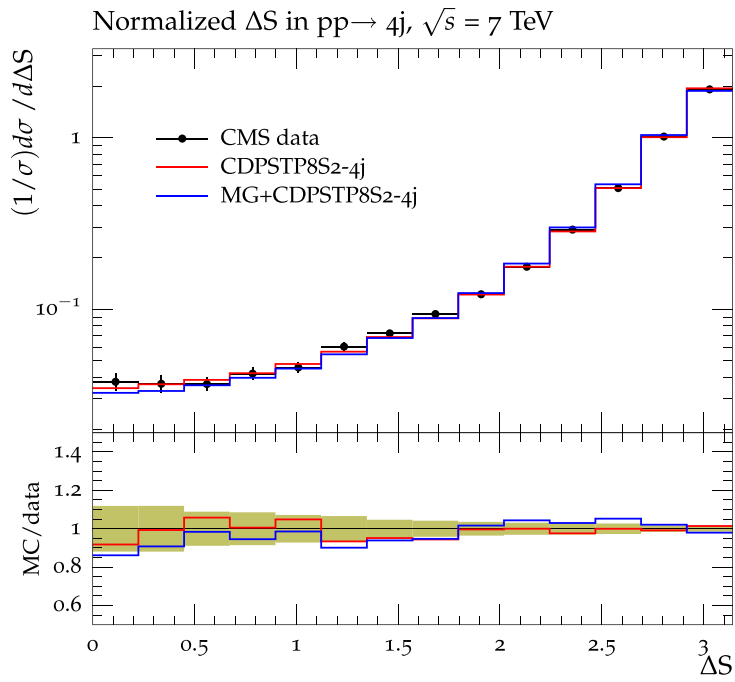

Normalized $\Delta^{\text {rel }} p_{\mathrm{T}}$ in $\mathrm{pp} \rightarrow 4 \mathrm{j}, \sqrt{s}=7 \mathrm{TeV}$

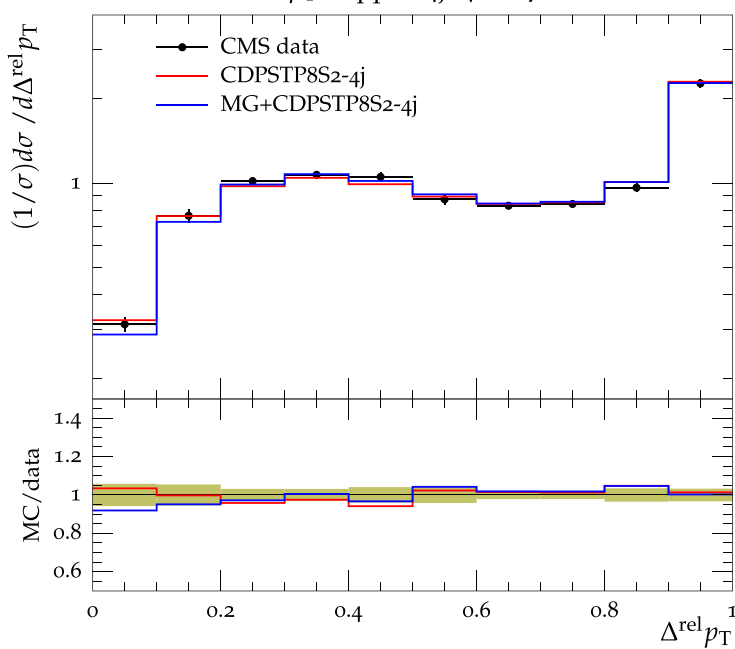

Fig. 8 Distributions in the correlation observables $\Delta \mathrm{S}($ top $)$ and $\Delta^{\text {rel }} p_{\mathrm{T}}$ (bottom) measured in four-jet production at $\sqrt{s}=7 \mathrm{TeV}$ [37], compared to predictions of PYTHIA8 using CDPSTP8S2-4j and of MADGRAPH (MG) interfaced to PYTHIA8 using CDPSTP8S2-4j (left) and PYTHIA8 using CUETP8M1 and HERWIG++ with CUETHppS1 (right).

Table 8 Values of $\sigma_{\text {eff }}$ at $\sqrt{s}=7 \mathrm{TeV}$ and $13 \mathrm{TeV}$ for CUETP8S1CTEQ6L1, CUETP8S1-HERAPDF1.5LO, and CUETP8M1, CUETHppS1, and for CDPSTP8S1-4j and CDPSTP8S2-4j. At $\sqrt{s}=7 \mathrm{TeV}$, also shown are the uncertainties in $\sigma_{\text {eff }}$ obtained from the eigentunes

\begin{tabular}{lll}
\hline CMS tune & $\sigma_{\text {eff }}(\mathrm{mb})$ at $7 \mathrm{TeV}$ & $\sigma_{\text {eff }}(\mathrm{mb})$ at $13 \mathrm{TeV}$ \\
\hline CUETP8S1-CTEQ6L1 & $27.8_{-1.3}^{+1.2}$ & $29.9_{-2.8}^{+1.6}$ \\
CUETP8S1-HERAPDF1.5LO & $29.1_{-2.0}^{+2.2}$ & $31.0_{-2.6}^{+3.8}$ \\
CUETP8M1 & $26.0_{-0.2}^{+0.6}$ & $27.9_{-0.4}^{+0.7}$ \\
CUETHppS1 & $15.2_{-0.6}^{+0.5}$ & $15.2_{-0.6}^{+0.5}$ \\
CDPSTP8S1-4j & $21.3_{-1.6}^{+1.2}$ & $21.8_{-0.7}^{+1.0}$ \\
CDPSTP8S2-4j & $19.0_{-3.0}^{+4.7}$ & $22.7_{-5.2}^{+10.0}$ \\
\hline
\end{tabular}
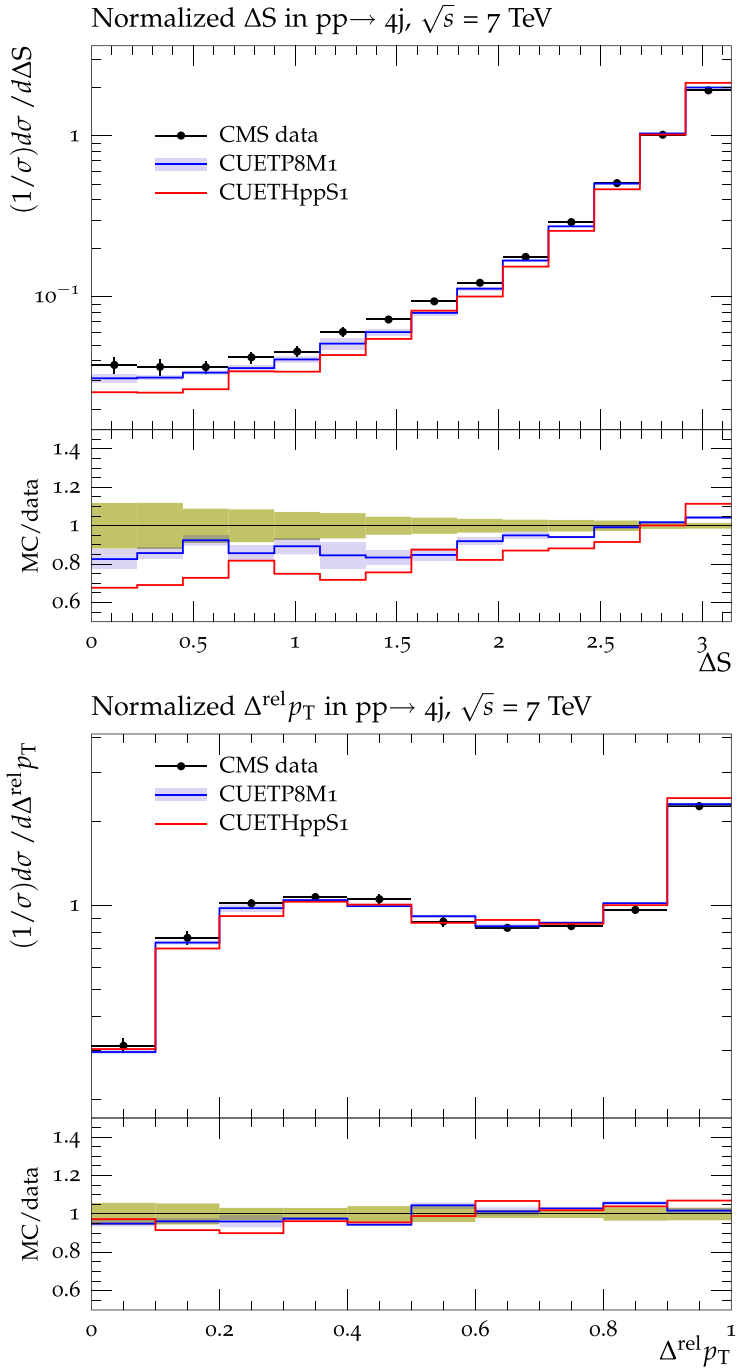

Also shown are the ratios of the predictions to the data. Predictions for CUETP8M1 (right) are shown with an error band corresponding to the total uncertainty obtained from the eigentunes (Appendix A). The green bands around unity represent the total experimental uncertainty

overlap function and, to a lesser extent, on the value of $p_{\mathrm{T} 0}$ in Eq. (2), and the range of the CR. We obtain two separate tunes for each channel: in the first one, we vary just the matteroverlap parameter expPow, to which the $\sigma_{\text {eff }}$ value is most sensitive, and in the second one, the whole set of parameters is varied. These two tunes allow to check whether the value of $\sigma_{\text {eff }}$ is stable relative to the choice of parameters.

The W+dijet and the four-jet channels are fitted separately. The fit to DPS-sensitive observables in the $\mathrm{W}+$ dijet channel gives a new determination of $\sigma_{\text {eff }}$ which can be compared to the value measured through the template method in the same final state [36]. Fitting the same way to the observables in the four-jet final state provides an estimate of $\sigma_{\text {eff }}$ for this channel. 

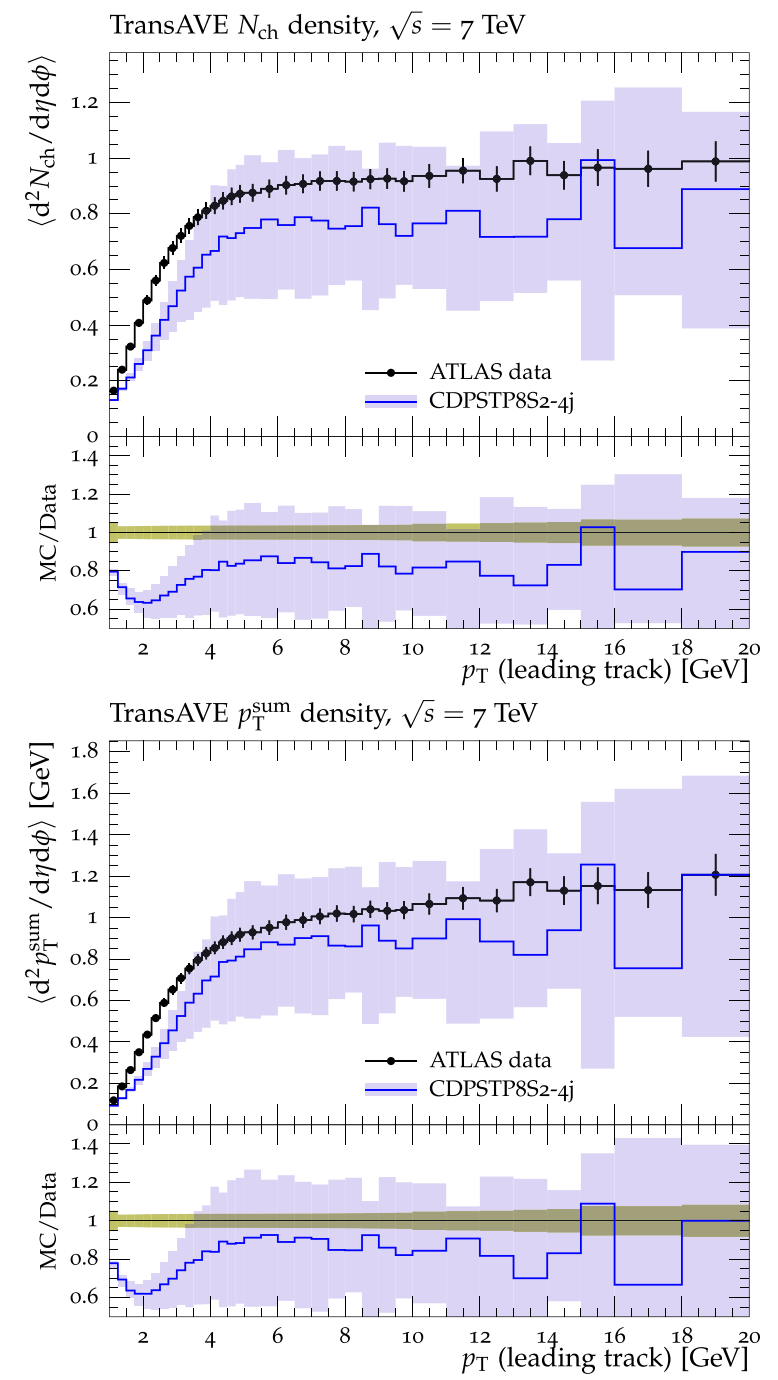

Fig. 9 ATLAS data at $\sqrt{s}=7 \mathrm{TeV}$ [39] for charged-particle (left) and $p_{\mathrm{T}}^{\text {sum }}$ densities (right) with $p_{\mathrm{T}}>0.5 \mathrm{GeV}$ and $|\eta|<$ 2.0 in the transverse (TransAVE) region compared to predictions of PYTHIA8 using CDPSTP8S2-4j (left) and CUETP8S1-CTEQ6L1, CUETP8S1-HERAPDF1.5LO, and CUETP8M1, plus HERWIG++ using

\subsection{Double-parton scattering in $\mathrm{W}+$ dijet production}

To study the dependence of the DPS-sensitive observables on MPI parameters, we construct two W+dijet DPS tunes, starting from the parameters of PYTHIA8 Tune 4C. In a partial tune only the parameter of the exponential distribution expPow is varied, and in a full tune all four parameters in Table 6 are varied. In a comparison of models with W+dijet events [36], it was shown that higher-order SPS contributions (not present in PYTHIA) fill a similar region of phase-space as the DPS signal. When such higher-order SPS diagrams are neglected, the measured DPS contribution to the W+dijet channel can be overestimated (i.e. $\sigma_{\text {eff }}$ underestimated). We therefore interface the LO matrix elements (ME) generated
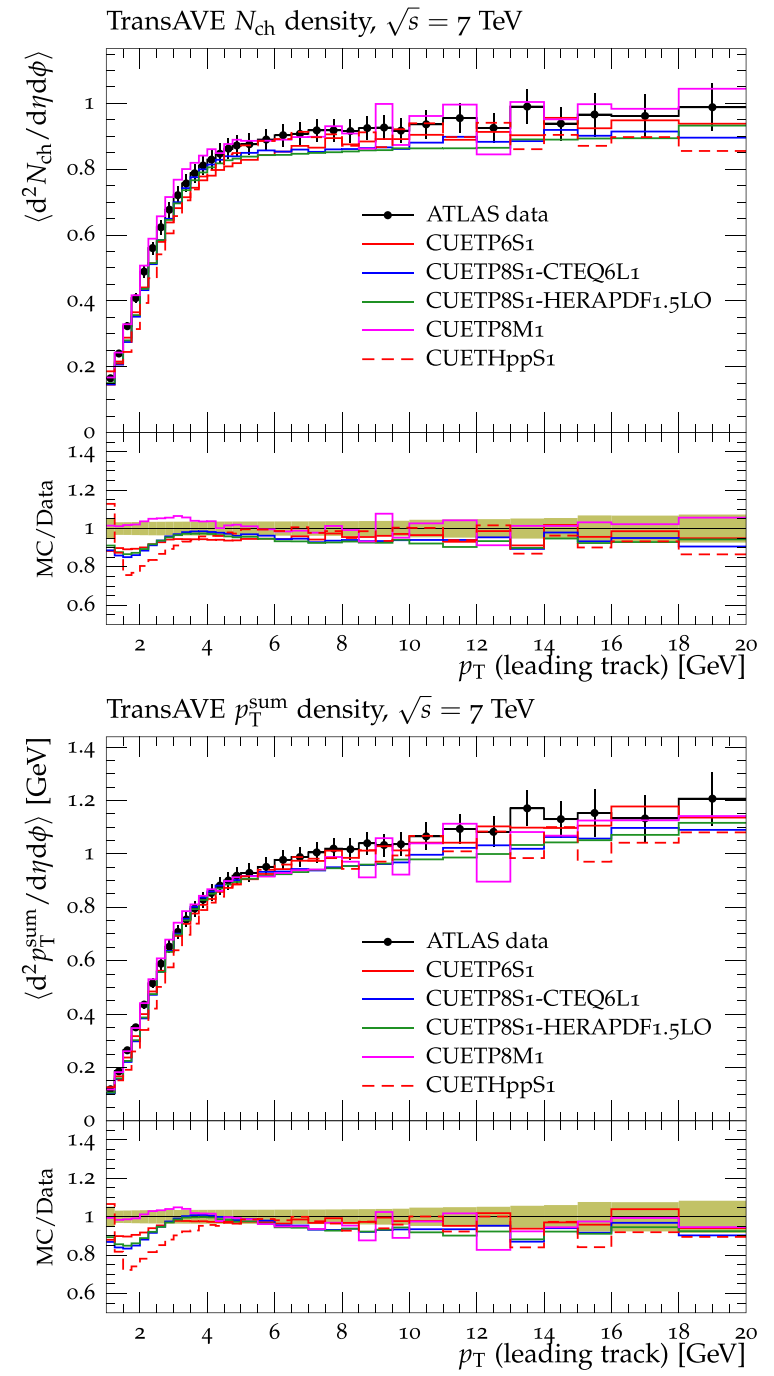

CUETHppS1 (right). The predictions of CDPSTP8S2-4j are shown with an error band corresponding to the total uncertainty obtained from the eigentunes (Appendix A). The bottom panels of each plot show the ratios of these predictions to the data, and the green bands around unity represent the total experimental uncertainty

by MADGRAPH 5 (version 1.5.14) [38] with PYTHIA8, and tune to the normalized distributions of the correlation observables in Eqs. (5) and (6). For this study, we produce MADGRAPH parton-level events with a W boson and up to four partons in the final state. The cross section is calculated using the CTEQ6L1 PDF with a matching scale for ME and parton shower (PS) jets set to $20 \mathrm{GeV}$. (In Sect. 4, we show that the CMS UE tunes can be interfaced to higher-order ME generators without additional tuning of MPI parameters). Figure 6 shows the CMS data [36] for the observables $\Delta \mathrm{S}$ and $\Delta^{\text {rel }} p_{\mathrm{T}}$ measured in $\mathrm{W}+$ dijet production, compared to predictions from MADGRAPH interfaced to PYTHIA8 Tune 4C, to Tune 4C with no MPI, to the partial CDPSTP8S1-Wj, as well as to the full CDPSTP8S2-Wj (CDPST stands for "CMS DPS 

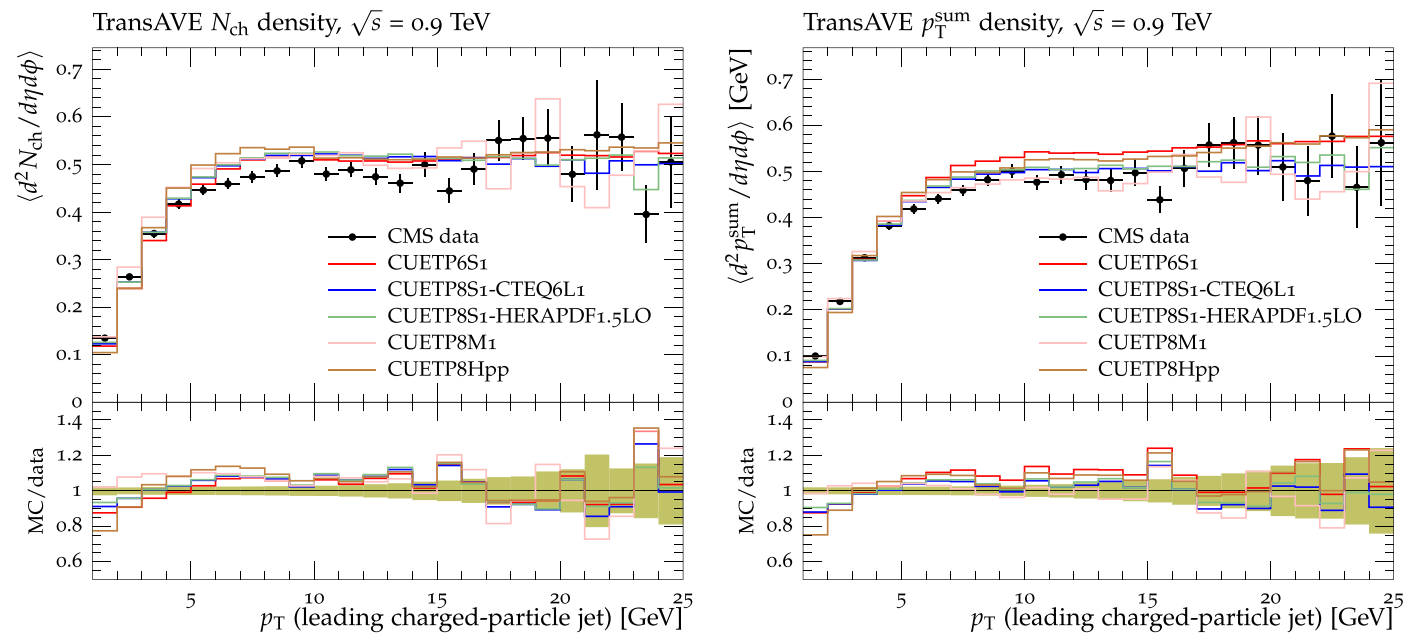

TransAVE $N_{\text {ch }}$ density, $\sqrt{s}=2.76 \mathrm{TeV}$
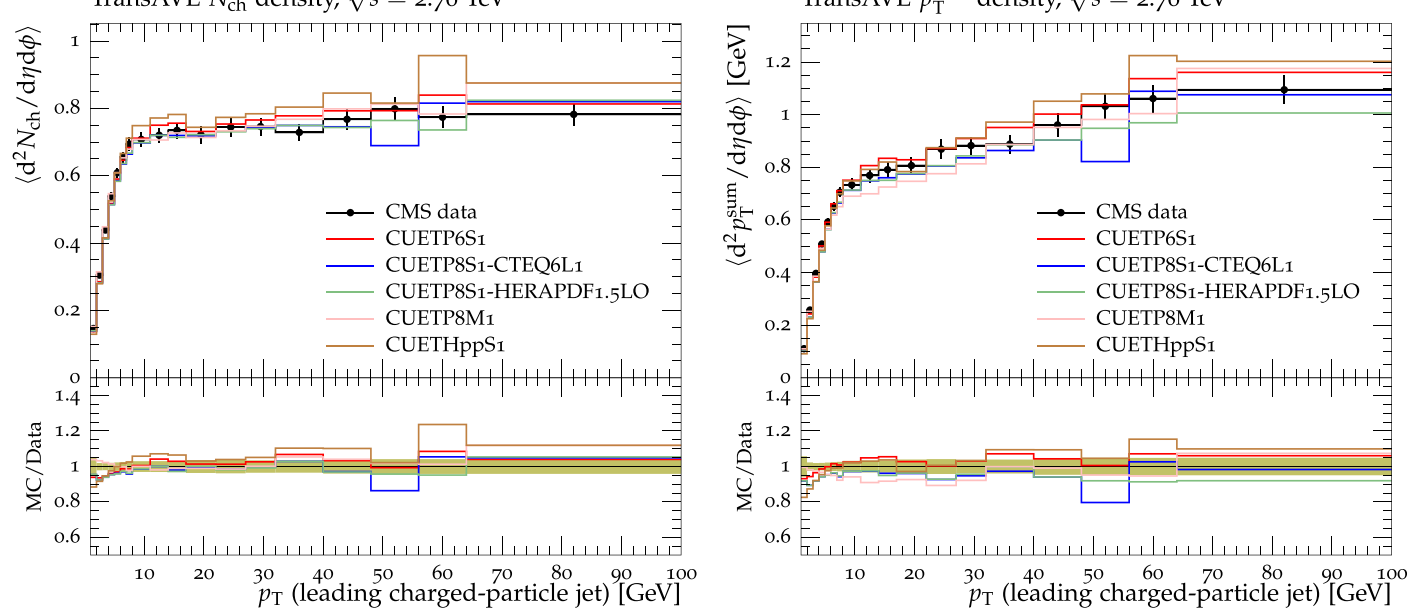

TransAVE $N_{\text {ch }}$ density, $\sqrt{s}=7 \mathrm{TeV}$

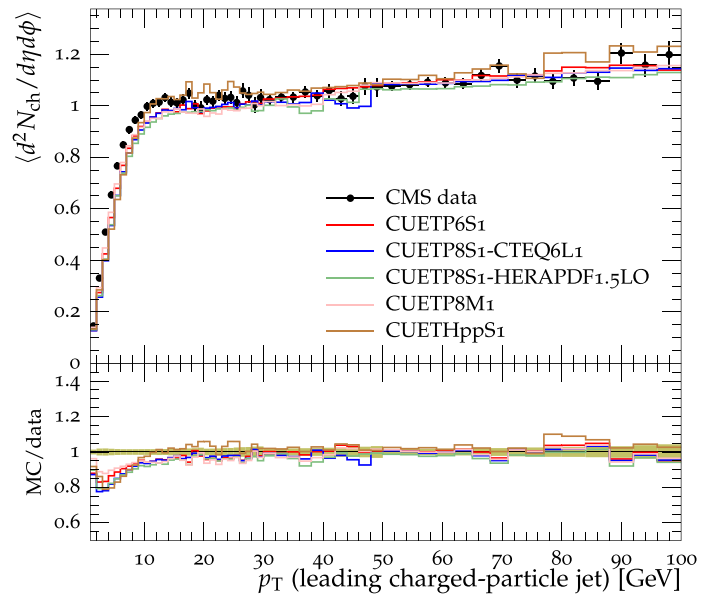

TransAVE $p_{\mathrm{T}}^{\text {sum }}$ density, $\sqrt{s}=7 \mathrm{TeV}$

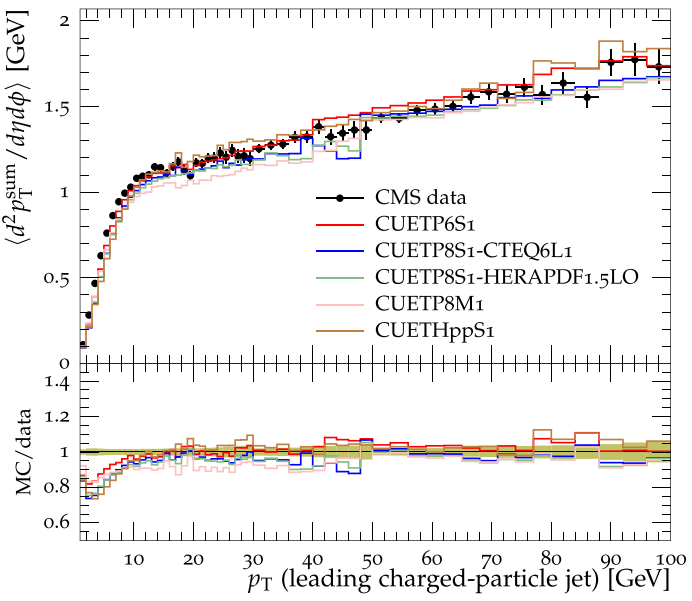

Fig. 10 CMS data on charged-particle (left) and $p_{\mathrm{T}}^{\text {sum }}$ (right) densities at $\sqrt{s}=0.9$ [24] (top), 2.76 [42] (middle), and $7 \mathrm{TeV}$ [24] (bottom) with $p_{\mathrm{T}}>0.5 \mathrm{GeV}$ and $|\eta|<2.0$ in the transverse (TransAVE) region as defined by the leading charged-particle jet, as a function of the transverse momentum of the leading charged-particle jet. The data

are compared to predictions of PYTHIA6 using CUETP6S1-CTEQ6L1, PYTHIA8 using CUETP8S1-CTEQ6L1, CUETP8S1-HERAPDF1.5LO, and CUETP8M1, and HERWIG++ using CUETHppS1. The bottom panels of each plot show the ratios of these predictions to the data, and the green bands around unity represent the total experimental uncertainty 

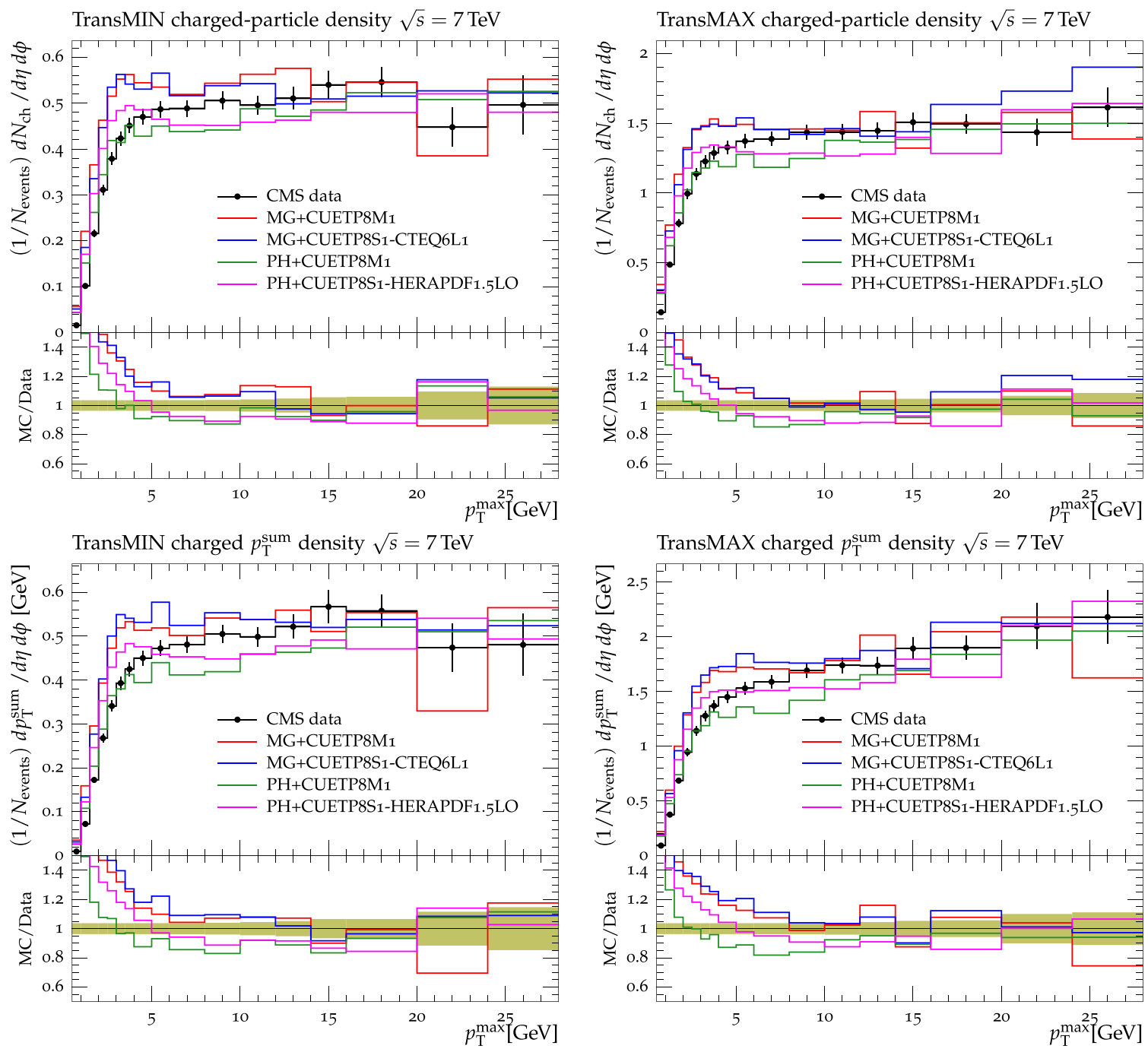

Fig. 11 CMS data at $\sqrt{s}=7 \mathrm{TeV}$ [17] for particle (top) and $p_{\mathrm{T}}^{\text {sum }} \mathrm{den}$ sities (bottom) for charged particles with $p_{\mathrm{T}}>0.5 \mathrm{GeV}$ and $|\eta|<0.8$ in the TransMIN (left) and TransMAX (right) regions, as defined by the leading charged particle, as a function of the transverse momentum of the leading charged-particle $p_{\mathrm{T}}^{\max }$. The data are compared to

MADGRAPH (MG), interfaced to PYTHIA8 using CUETP8S1-CTEQ6L1 and CUETP8M1, and to POWHEG (PH), interfaced to PYTHIA8 using CUETP8S1-HERAPDF1.5LO and CUETP8M1. The bottom panels of each plot show the ratios of these predictions to the data, and the green bands around unity represent the total experimental uncertainty

tune"). Table 6 gives the best-fit parameters and the resulting $\sigma_{\text {eff }}$ values at $\sqrt{s}=7 \mathrm{TeV}$. The uncertainties quoted for $\sigma_{\text {eff }}$ are computed from the uncertainties of the fitted parameters given by the eigentunes. For Tune $4 \mathrm{C}$, the uncertainty in $\sigma_{\text {eff }}$ is not provided since no eigentunes are available for that tune. The resulting values of $\sigma_{\text {eff }}$ are compatible with the value measured by CMS using the template method of $\sigma_{\text {eff }}=20.6 \pm 0.8$ (stat) \pm 6.6 (syst) $\mathrm{mb}[36]$.

\subsection{Double-parton scattering in four-jet production}

Starting from the parameters of PYTHIA8 Tune 4C, we construct two different four-jet DPS tunes. As in the W+dijet channel, in the partial tune just the exponential-dependence

parameter, expPow, while in the full tune all four parameters of Table 7 are varied. We obtain a good fit to the four-jet data without including higher-order ME contributions. However, we also obtain a good fit when higher-order (real) ME terms are generated with MADGRAPH. In Figs. 7 and 8 the correlation observables $\Delta \mathrm{S}$ and $\Delta{ }^{\text {rel }} p_{\mathrm{T}}$ in four-jet production [37] are compared to predictions obtained with PYTHIA8 Tune 4C, Tune 4C without MPI, CDPSTP8S1-4j, CDPSTP8S2-4j, and MADGRAPH interfaced to CDPSTP8S2-4j. Table 7 gives the best-fit parameters and the resulting $\sigma_{\text {eff }}$ values. The values of $\sigma_{\text {eff }}$ extracted from the CMS PYTHIA8 DPS tunes give the first determination of $\sigma_{\text {eff }}$ in four-jet production at $\sqrt{s}=7 \mathrm{TeV}$. The uncertainties quoted for $\sigma_{\text {eff }}$ are obtained from the eigentunes. 

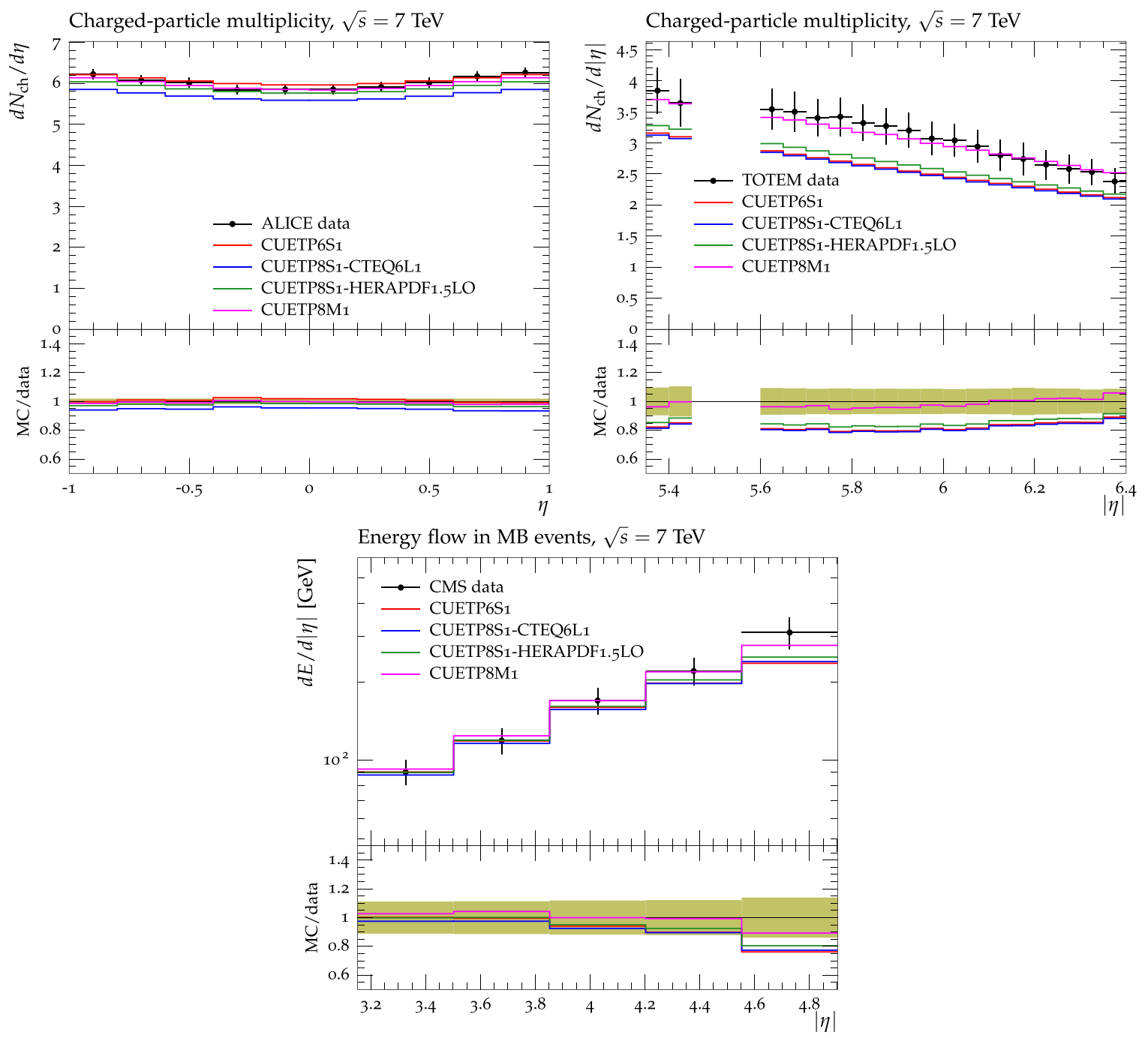

Fig. 12 ALICE data at $\sqrt{s}=7 \mathrm{TeV}$ [47] for the charged-particle pseudorapidity distribution, $\mathrm{dN}_{\mathrm{ch}} / \mathrm{d} \eta$, in inclusive inelastic pp collisions (top left). TOTEM data at $\sqrt{s}=7 \mathrm{TeV}$ [48] for the chargedparticle pseudorapidity distribution, $\mathrm{dN}_{\mathrm{ch}} / \mathrm{d} \eta$, in inclusive inelastic pp collisions $\left(p_{\mathrm{T}}>40 \mathrm{MeV}, \mathrm{N}_{\mathrm{chg}} \geq 1\right)$ (top right). CMS data at $\sqrt{s}=7 \mathrm{TeV}$ [50] for the energy flow $\mathrm{d} E / \mathrm{d} \eta$, in MB pp collisions.

\section{Validation of CMS tunes}

Here we discuss the compatibility of the UE and DPS tunes. In addition, we compare the CMS UE tunes with UE data that have not been used in the fits, and we examine how well Drell-Yan and MB observables can be predicted from MC simulations using the UE tunes. We also show that the CMS UE tunes can be interfaced to higher-order ME generators without additional tuning of the MPI parameters.

\subsection{Compatibility of UE and DPS tunes}

The values of $\sigma_{\text {eff }}$ obtained from simulations applying the CMS PYTHIA8 UE and DPS tunes at $\sqrt{s}=7 \mathrm{TeV}$
The data are compared to PYTHIA6 using CUETP6S1-CTEQ6L1, and to PYTHIA8 using CUETP8S1-CTEQ6L1, CUETP8S1-HERAPDF1.5LO, and CUETP8M1. The bottom panels of each plot show the ratios of these predictions to the data, and the green bands around unity represent the total experimental uncertainty

and $\sqrt{s}=13 \mathrm{TeV}$ are listed in Table 8 . The uncertainties, obtained from eigentunes are also quoted in Table 8 . At $\sqrt{s}=7 \mathrm{TeV}$, the CMS DPS tunes give values of $\sigma_{\text {eff }} \approx 20 \mathrm{mb}$, while the CMS PYTHIA8 UE tunes give slightly higher values in the range $26-29 \mathrm{mb}$ as shown in Figs. 8 and 9. Figure 8 shows the CMS DPS-sensitive data for four-jet production at $\sqrt{s}=7 \mathrm{TeV}$ compared to predictions using CDPSTP8S2-4j, CUETP8M1, and CUETHppS1. Figure 9 shows ATLAS UE data at $\sqrt{s}=$ $7 \mathrm{TeV}$ [39] compared to predictions obtained with various tunes: CDPSTP8S2-4j with uncertainty bands, CUETP6S1CTEQ6L1, CUETP8S1-CTEQ6L1, CUETP8S1HERAPDF1.5LO, CUETP8M1, and CUETHppS1. Predictions from PYTHIA8 using CUETP8M1 describe reasonably 


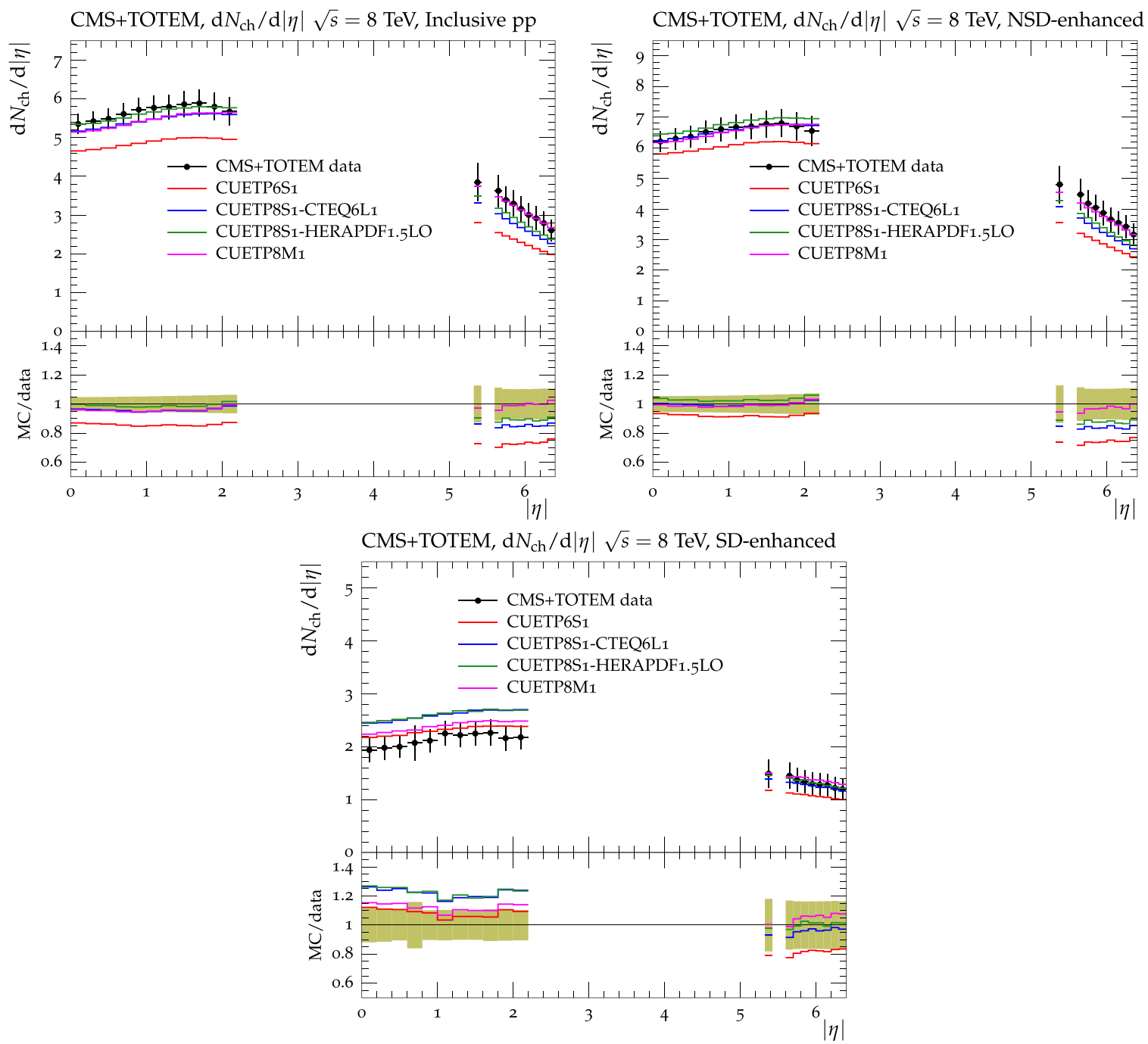

Fig. 13 Combined CMS and TOTEM data at $\sqrt{s}=8 \mathrm{TeV}$ [50] for the charged-particle distribution $\mathrm{dN}_{\mathrm{ch}} / \mathrm{d} \eta$, in inclusive inelastic (top left), NSD-enhanced (top right), and SD-enhanced (bottom) pp collisions. The data are compared to PYTHIA6 using CUETP6S1-CTEQ6L1, and to
PYTHIA8 using CUETP8S1-CTEQ6L1, CUETP8S1-HERAPDF1.5LO, and CUETP8M1. The bottom panels of each plot show the ratios of these predictions to the data, and the green bands around unity represent the total experimental uncertainty well the DPS observables, but do not fit them as well as predictions using the DPS tunes. On the other hand, predictions using CDPSTP8S2-4j do not fit the UE data as well as the UE tunes do.

As discussed previously, the PYTHIA8 tunes use a single exponential matter-overlap function, while the HERWIG++ tune uses a matter-overlap function that is related to the Fourier transform of the electromagnetic form factor. The CUETHppS1 gives a value of $\sigma_{\text {eff }} \approx 15 \mathrm{mb}$, while UE and DPS tunes give higher values of $\sigma_{\text {eff }}$. It should be noted that $\sigma_{\text {eff }}$ is a parton-level observable and its importance is not in the modelled value of $\sigma_{\text {eff }}$, but in what is learned about the transverse proton profile (and its energy evolution), and how well the models describe the DPS-sensitive observables. As can be seen in Fig. 8, predictions using CUETP8M1 describe the DPS-sensitive observables better than CUETHppS1, but not quite as well as the DPS tunes. We performed a simultaneous PYTHIA8 tune that included both the UE data and DPSsensitive observables, however, the quality of the resulting fit was poor. This confirms the difficulty of describing soft and hard MPI within the current PYTHIA and HERWIG++ frameworks. Recent studies $[40,41]$ suggest the need for introducing parton correlation effects in the MPI framework in order to achieve a consistent description of both the UE and DPS observables.

\subsection{Comparisons with other UE measurements}

Figure 10 shows charged particle and $p_{\mathrm{T}}^{\text {sum }}$ densities [24,42] at $\sqrt{s}=0.9,2.76$, and $7 \mathrm{TeV}$ with $p_{\mathrm{T}}>0.5 \mathrm{GeV}$ and $|\eta|<$ 

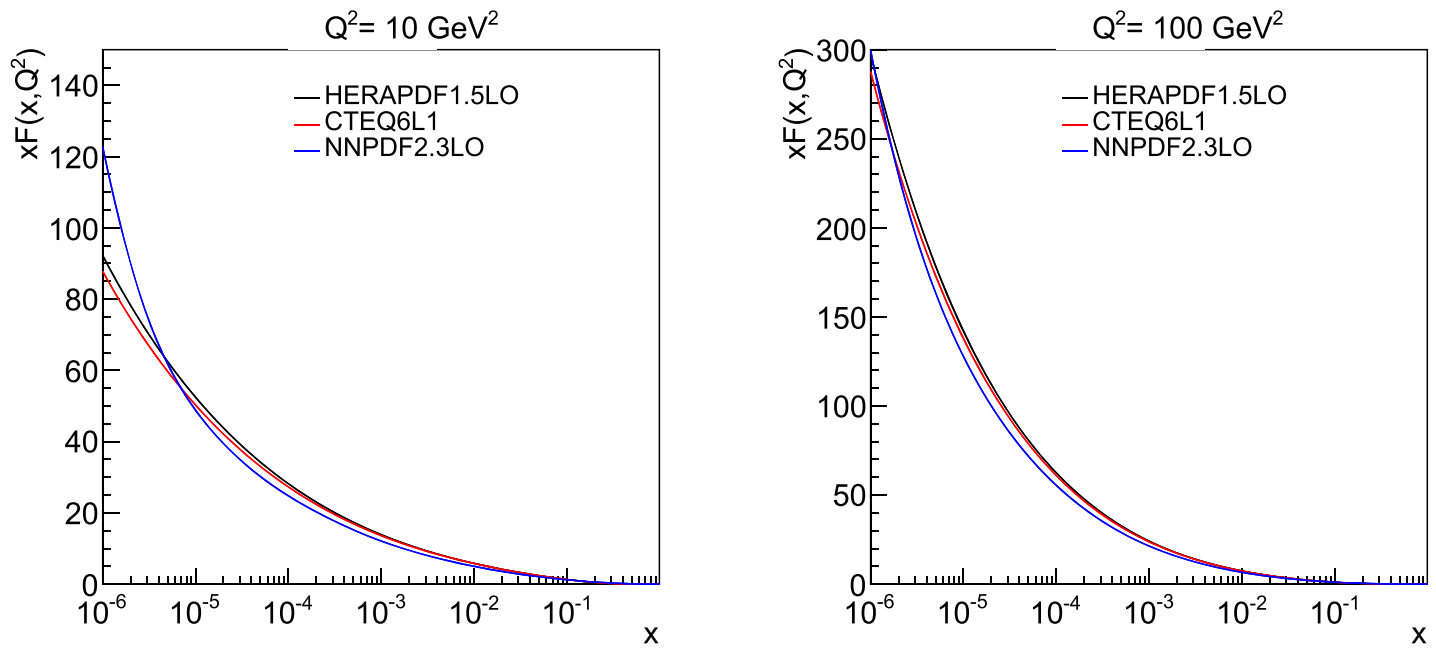

Fig. 14 Comparison of gluon distributions in the proton for the CTEQ6L1, HERAPDF1.5LO, and NNPDF2.3LO PDF sets, at the $Q^{2}=10$ GeV ${ }^{2}$ (left) and $100 \mathrm{GeV}^{2}$ (right)

2.0 in the TransAVE region, as defined by the leading jet reconstructed by using just the charged particles (also called "leading track-jet") compared to predictions using the CMS UE tunes. The CMS UE tunes describe quite well the UE measured using the leading charged particle as well as the leading charged-particle jet.

Tunes obtained from fits to UE data and combined with higher-order ME calculations [43] can also be cross-checked against the data. The CMS UE tunes can be interfaced to higher-order ME generators without spoiling their good description of the UE. In Fig. 11, the charged-particle and $p_{\mathrm{T}}^{\text {sum }}$ densities in the TransMIN and TransMAX regions as a function of $p_{\mathrm{T}}^{\max }$, are compared to predictions obtained with MADGRAPH and POWHEG $[44,45]$ interfaced to PYTHIA8 using CUETP8S1-CTEQ6L1 and CUETP8M1. In MADGRAPH, up to four partons are simulated in the final state. The cross section is calculated with the CTEQ6L1 PDF. The ME/PS matching scale is taken to be $10 \mathrm{GeV}$. The POWHEG predictions are based on next-to-leading-order (NLO) dijet using the CT10nlo PDF [46] interfaced to PYTHIA8 based on CUETP8M1, and HERAPDF1.5NLO [21] interfaced to the PYTHIA8 using CUETP8S1-HERAPDF1.5LO.

The poor agreement below $p_{\mathrm{T}}^{\max }=5 \mathrm{GeV}$ in Fig. 11 is not relevant as the minimum $\hat{p}_{\mathrm{T}}$ for MADGRAPH and POWHEG is $5 \mathrm{GeV}$. The agreement with the UE data in the plateau region of $p_{\mathrm{T}}^{\max }>5 \mathrm{GeV}$ is good. All these figures show that CMS UE tunes interfaced to higher-order ME generators do not spoil their good description of the UE data.

\subsection{Predicting MB observables}

The UE is studied in events containing a hard scatter, whereas most of the MB collisions are softer and can include diffrac- tive scatterings. It is however interesting to see how well predictions based on the CMS UE tunes can describe the properties of MB distributions. Figure 12 shows predictions using CMS UE tunes for the ALICE [47] and TOTEM data [48] at $\sqrt{s}=7 \mathrm{TeV}$ for the charged-particle pseudorapidity distribution, $\mathrm{dN}_{\mathrm{ch}} / \mathrm{d} \eta$, and for $\mathrm{d} E / \mathrm{d} \eta$ [49] at $\sqrt{s}=7 \mathrm{TeV}$. These observables are sensitive to single-diffraction dissociation, central-diffraction, and double-diffraction dissociation, which are modelled in PYTHIA. Since HERWIG++ does not include a model for single-diffraction dissociation, centraldiffraction, and double-diffraction dissociation, we do not show it here. Figure 13 shows predictions using the CMS UE tunes for the combined CMS+TOTEM data at $\sqrt{s}=$ $8 \mathrm{TeV}$ [50] for the charged-particle pseudorapidity distribution, $\mathrm{dN}_{\mathrm{ch}} / \mathrm{d} \eta$, for inelastic, non single-diffraction-enhanced, and single-diffraction-enhanced proton-proton collisions.

The PYTHIA8 event generator using the UE tunes describes the MB data better than PYTHIA6 with the UE tune, which is likely due to the improved modelling of single-diffraction dissociation, central-diffraction, and double-diffraction dissociation in PYTHIA8. Predictions with all the UE tunes describe fairly well $\mathrm{MB}$ observables in the central region $(|\eta|<2)$, however, only predictions obtained with CUETP8M1 describe the data in the forward region $(|\eta|>$ 4). This is due to the PDF used in CUETP8M1. As can be seen in Fig. 14, the NNPDF2.3LO PDF at scales $Q^{2}$ $=10 \mathrm{GeV}^{2}$ (corresponding to hard scatterings with $\hat{p}_{\mathrm{T}} \sim$ $3 \mathrm{GeV}$ ) and small $x$, features a larger gluon density than in CTEQ6L1 and HERAPDF1.5LO, thereby contributing to more particles (and more energy) produced in the forward region. We have checked that increasing the gluon distribution in HERAPDF1.5LO at values below $10^{-5}$ improved the description of the charged-particle multiplicity measurements in the forward region. 
CMS, Inclusive jets, $\sqrt{s}=7 \mathrm{TeV}, 0.0<|y|<0.5$

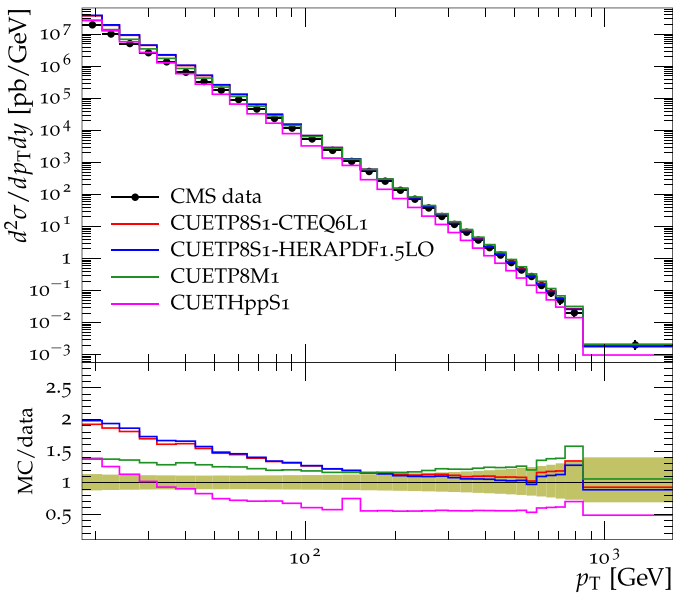

CMS, Inclusive jets, $\sqrt{s}=7 \mathrm{TeV}, 1.0<|y|<1.5$

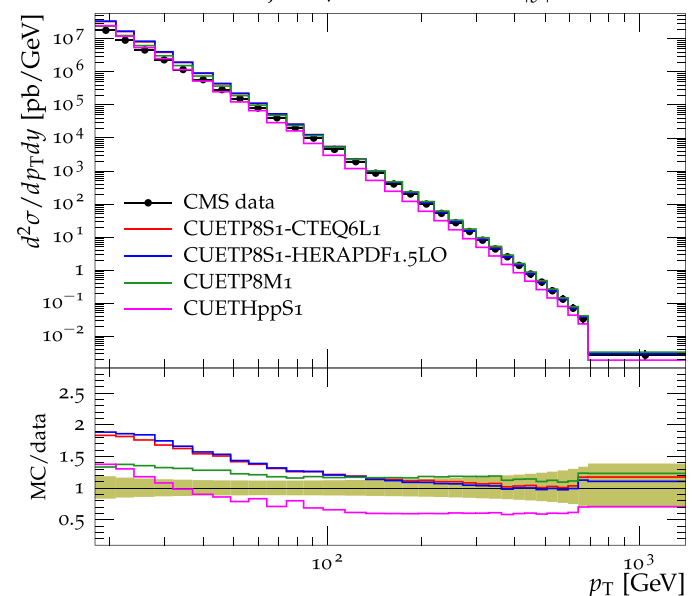

CMS, Inclusive jets, $\sqrt{s}=7 \mathrm{TeV}, 2.0<|y|<2.5$

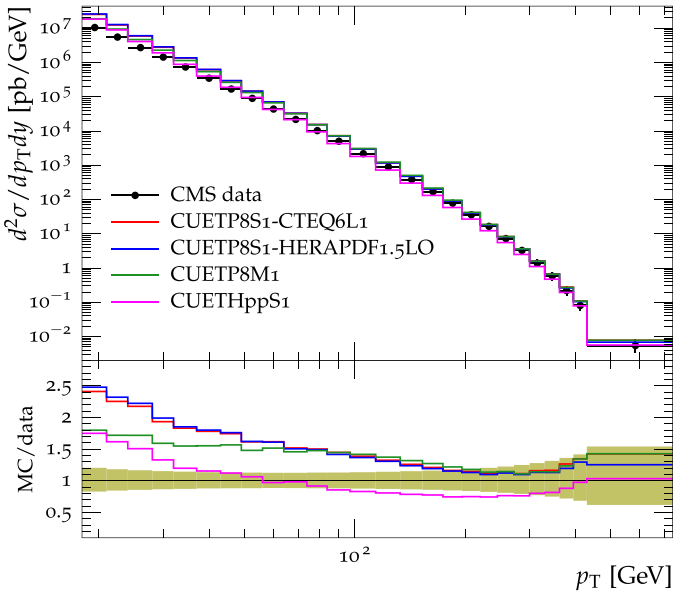

Fig. 15 CMS data at $\sqrt{s}=7 \mathrm{TeV}$ [51] for the inclusive jet cross section as a function of $p_{\mathrm{T}}$ in different rapidity ranges compared to predictions of PYTHIA8 using CUETP8S1-CTEQ6L1, CUETP8S1HERAPDF, and CUETP8M1, and of HERWIG++ using CUETHppS1.
CMS, Inclusive jets, $\sqrt{s}=7 \mathrm{TeV}, 0.5<|y|<1.0$

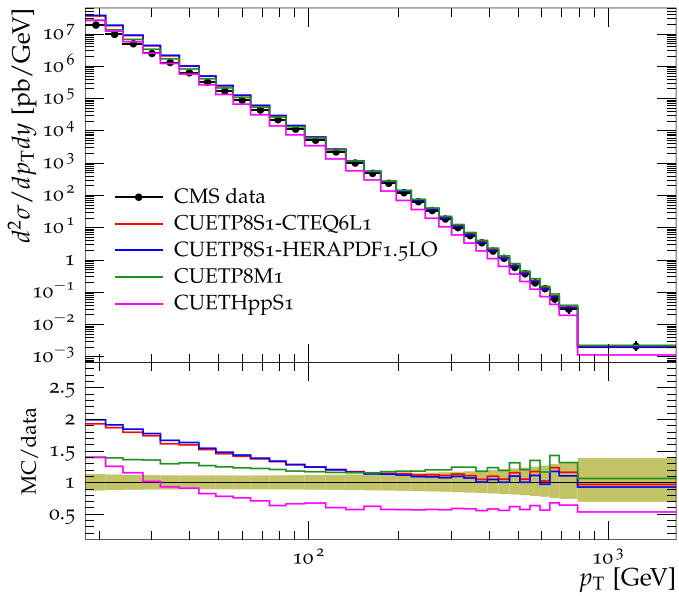

CMS, Inclusive jets, $\sqrt{s}=7 \mathrm{TeV}, 1.5<|y|<2.0$

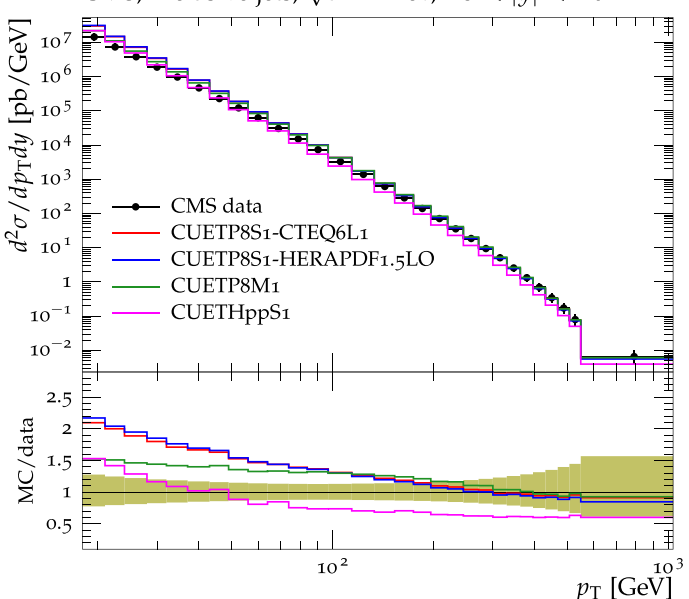

CMS, Inclusive jets, $\sqrt{s}=7 \mathrm{TeV}, 2.5<|y|<3.0$

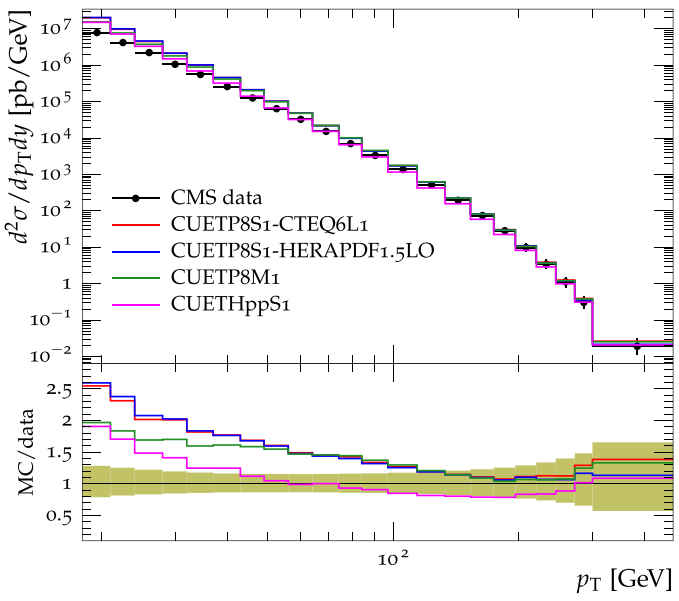

The bottom panels of each plot show the ratios of these predictions to the data, and the green bands around unity represent the total experimental uncertainty 
CMS, Inclusive jets, $\sqrt{s}=7 \mathrm{TeV}, 0.0<|y|<0.5$

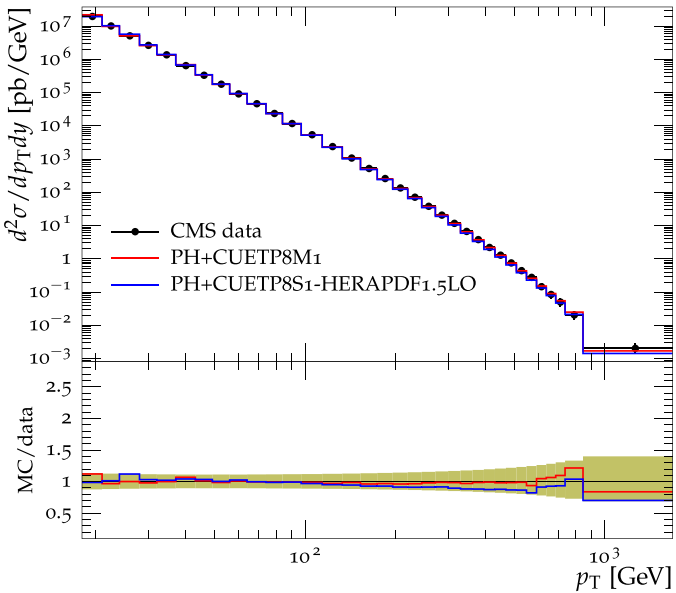

CMS, Inclusive jets, $\sqrt{s}=7 \mathrm{TeV}, 1.0<|y|<1.5$

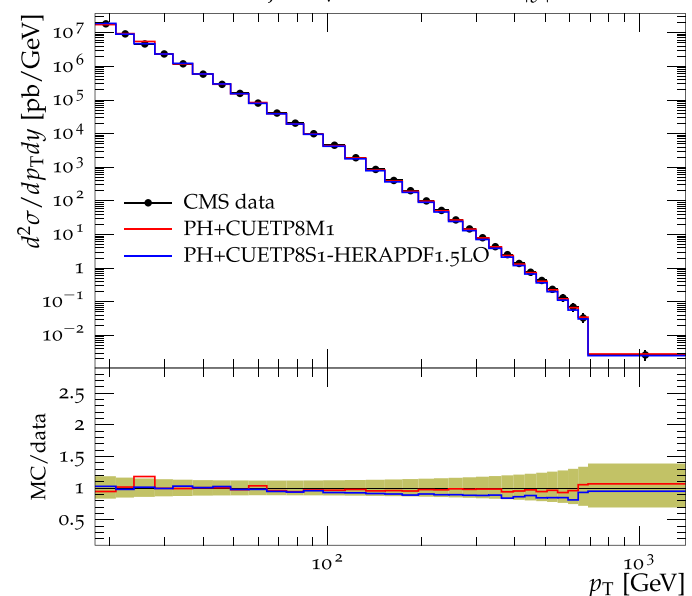

CMS, Inclusive jets, $\sqrt{s}=7 \mathrm{TeV}, 2.0<|y|<2.5$

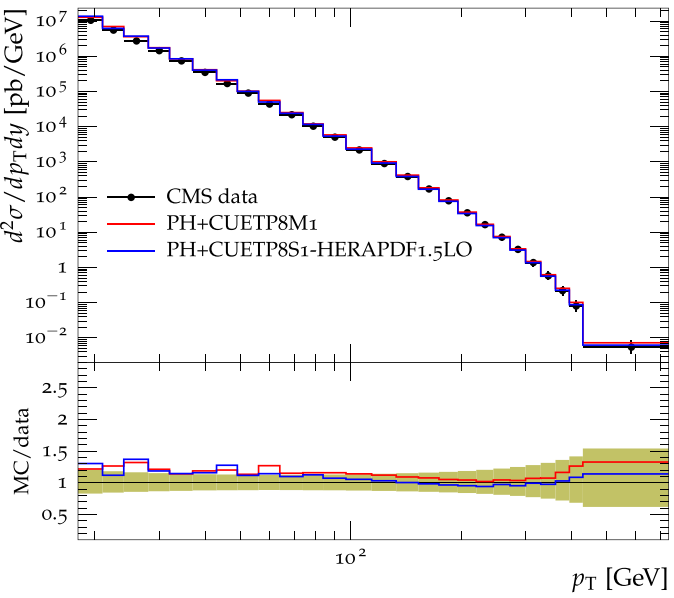

Fig. 16 CMS data at $\sqrt{s}=7 \mathrm{TeV}$ [51] for the inclusive jet cross section as a function of $p_{\mathrm{T}}$ in different rapidity ranges compared to predictions of POWHEG interfaced to PYTHIA8 using CUETP8S1-
CMS, Inclusive jets, $\sqrt{s}=7 \mathrm{TeV}, 0.5<|y|<1.0$

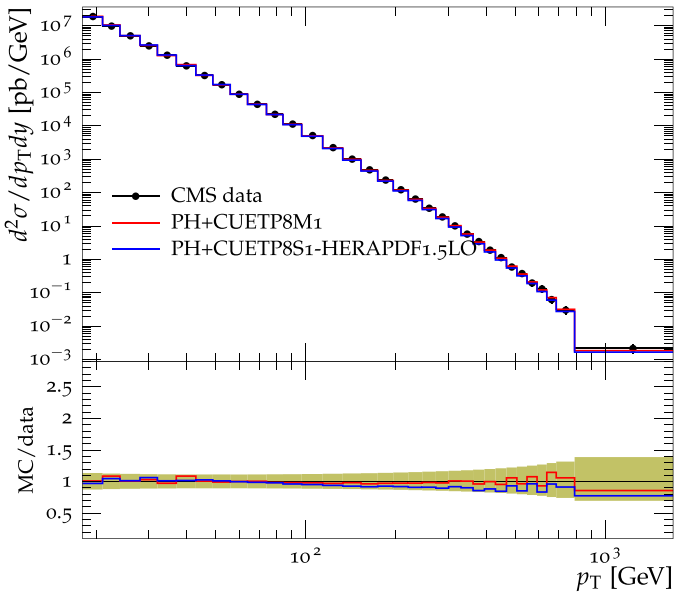

CMS, Inclusive jets, $\sqrt{s}=7 \mathrm{TeV}, 1.5<|y|<2.0$

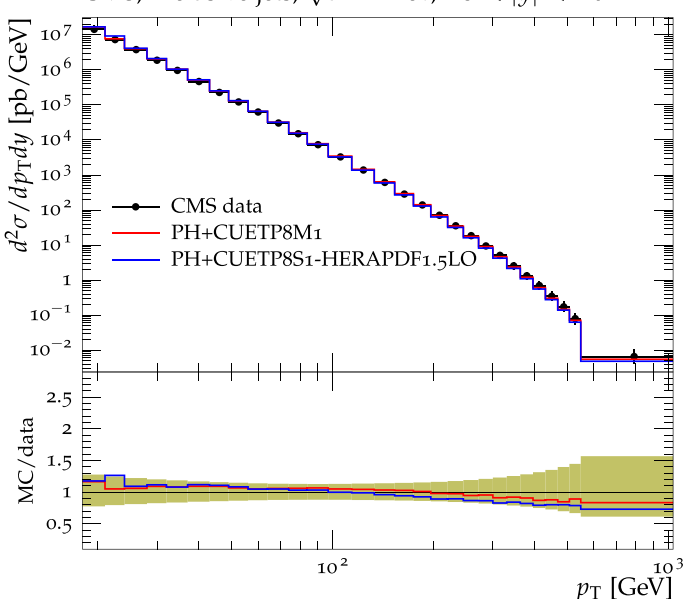

CMS, Inclusive jets, $\sqrt{s}=7 \mathrm{TeV}, 2.5<|y|<3.0$

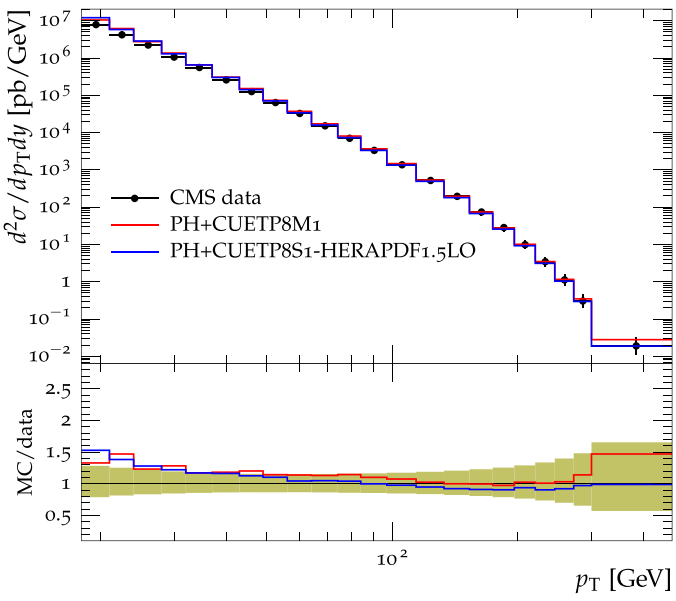

HERAPDF1.5LO and CUETP8M1. The bottom panels of each plot show the ratios of these predictions to the data, and the green bands around unity represent the total experimental uncertainty 


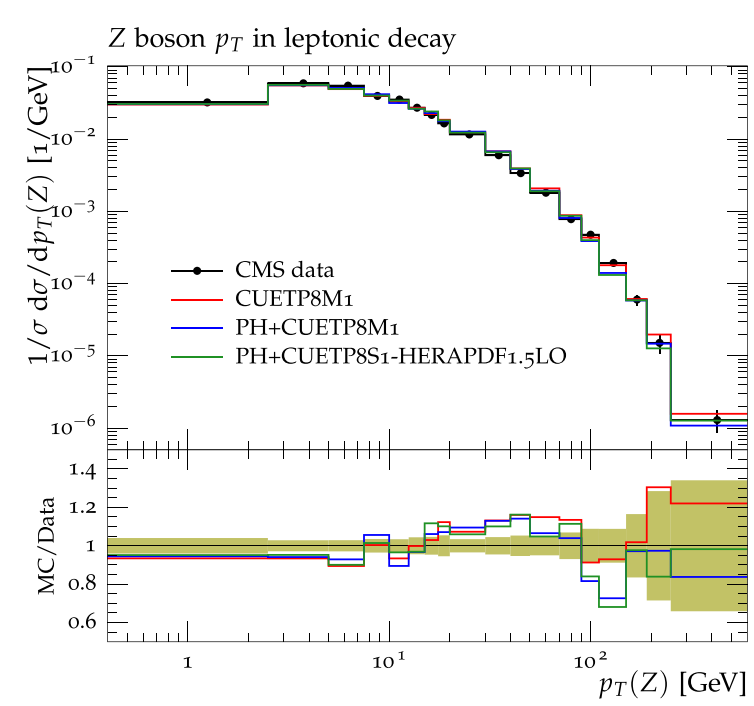

Fig. 17 Transverse momentum $p_{\mathrm{T}}$ (left) and rapidity distributions (right) of $\mathrm{Z}$ boson production in pp collisions at $\sqrt{s}=7 \mathrm{TeV}$ [52]. The data are compared to PYTHIA8 using CUETP8M1, and to POWHEG

\subsection{Comparisons with inclusive jet production}

In Fig. 15 predictions using CUETP8S1-CTEQ6L1, CUETP8S1-HERAPDF1.5LO, and CUETP8M1, and CUETHppS1 are compared to inclusive jet cross section at $\sqrt{s}=7 \mathrm{TeV}$ [51] in several rapidity ranges. Predictions using CUETP8M1 describe the data best, however, all the tunes overshoot the jet spectra at small $p_{\mathrm{T}}$. Predictions from the CUETHppS1 underestimate the high $p_{\mathrm{T}}$ region at central rapidity $(|y|<2.0)$. In Fig. 16, the inclusive jet cross sections are compared to predictions from POWHEG interfaced to PYTHIA8 using CUETP8S1-HERAPDF1.5LO and CUETP8M1. A very good description of the measurement is obtained.

\subsection{Comparisons with $\mathrm{Z}$ boson production}

In Fig. 17 the $p_{\mathrm{T}}$ and rapidity distributions of the $\mathrm{Z}$ boson in pp collisions at $\sqrt{s}=7 \mathrm{TeV}$ [52] are shown and compared to PYTHIA8 using CUETP8M1, and to POWHEG interfaced to PYTHIA8 using CUETP8S1-CTEQ6L1 and CUETP8M1. The prediction using PYTHIA8 with CUETP8M1 (without POWHEG) agrees reasonably well with the distribution of the $\mathrm{Z}$ boson at small $p_{\mathrm{T}}$ values. Also, when interfaced to POWHEG, which implements an inclusive $\mathrm{Z}$ boson NLO calculation, the agreement is good over the whole spectrum.

In Fig. 18 the charged-particle and $p_{\mathrm{T}}^{\text {sum }}$ densities [26] in the toward, away, and transverse (TransAVE) regions as defined by the $\mathrm{Z}$ boson in proton-proton collisions at $\sqrt{s}=7 \mathrm{TeV}$ are compared to predictions of PYTHIA8 using CUETP8M1. Also shown are MADGRAPH and POWHEG

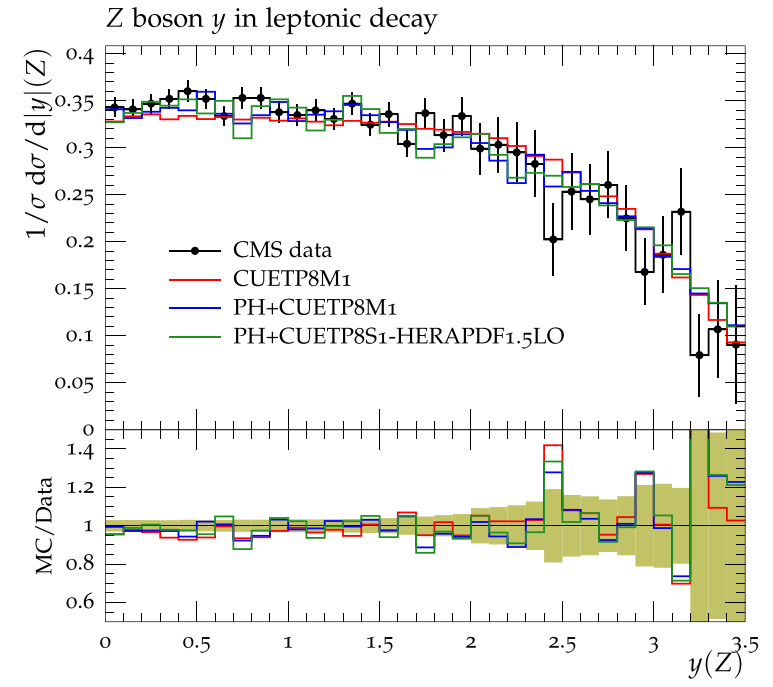

interfaced to PYTHIA8 using CUETP8S1-CTEQ6L1 and CUETP8M1. The green bands in the ratios represent the total experimental uncertainty

results interfaced to PYTHIA8 using CUETP8S1HERAPDF1.5LO and CUETP8M1. The MADGRAPH generator simulates Drell-Yan events with up to four partons, using the CTEQ6L1 PDF. The matching of ME partons and PS is performed at a scale of $20 \mathrm{GeV}$. The POWHEG events are obtained using NLO inclusive Drell-Yan production, including up to one additional parton. The POWHEG events are interfaced to PYTHIA8 using CUETP8M1 and CUETP8S1HERAPDF1.5LO. The predictions based on CUETP8M1 do not fit the $\mathrm{Z}$ boson data unless they are interfaced to a higher-order ME generator. In PYTHIA8 only the Born term ( $\mathrm{q} \overline{\mathrm{q}} \rightarrow \mathrm{Z}$ ), corrected for single-parton emission, is generated. This ME configuration agrees well with the observables in the away region in data, when the $\mathrm{Z}$ boson recoils against one or more jets. In the transverse and toward regions, larger discrepancies between data and PYTHIA8 predictions appear at high $p_{\mathrm{T}}$, where the occurrence of multijet emission has a large impact. To describe $\mathrm{Z}$ boson production at $\sqrt{s}=7 \mathrm{TeV}$ in all regions, higher-order contributions (starting with Z+2-jets), as used in interfacing PYTHIA to POWHEG or MADGRAPH, must be included.

\section{Extrapolation to $13 \mathrm{TeV}$}

In this section, predictions at $\sqrt{s}=13 \mathrm{TeV}$, based on the new tunes, for observables sensitive to the UE are presented. Figure 19 shows the predictions at $13 \mathrm{TeV}$ for the chargedparticle and the $p_{\mathrm{T}}^{\text {sum }}$ densities in the TransMIN, TransMAX, and TransDIF regions, as defined by the leading charged particle as a function of $p_{\mathrm{T}}^{\max }$ based on the five new CMS 

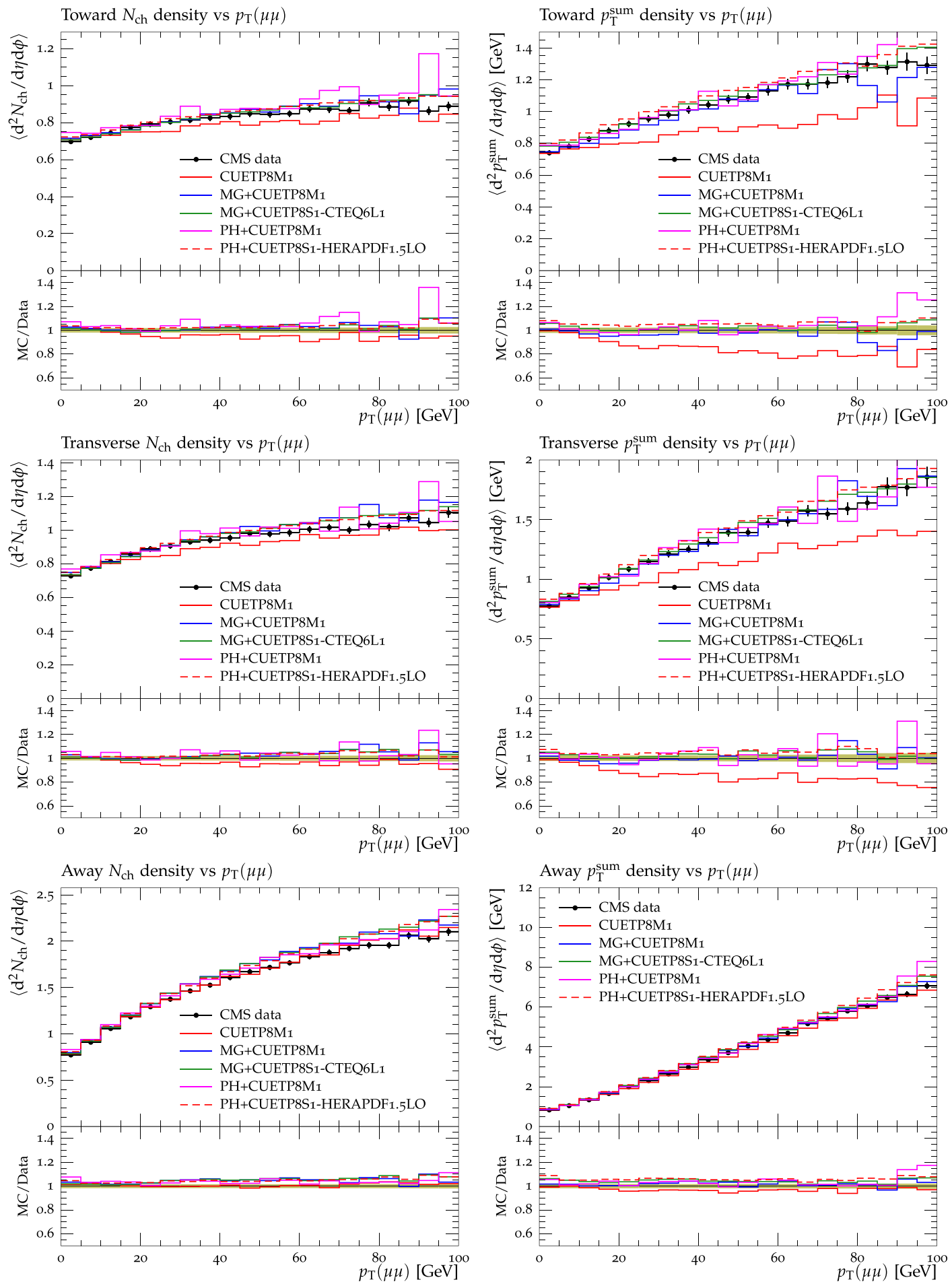

Fig. 18 Charged-particle (left) and $p_{\mathrm{T}}^{\text {sum }}$ densities (right) in the toward (top), away (middle), and transverse (TransAVE) (bottom) regions, as defined by the Z-boson direction in Drell-Yan production at $\sqrt{s}=$ $7 \mathrm{TeV}$ [26]. The data are compared to PYTHIA8 using CUETP8M1, to

MADGRAPH (MG) interfaced to PYTHIA8 using CUETP8S1-CTEQ6L1 and CUETP8M1, and to POWHEG (PH) interfaced to PYTHIA8 using CUETP8S1-HERAPDF1.5LO and CUETP8M1. The green bands in the ratios represent the total experimental uncertainty 
TransMIN charged-particle density $\sqrt{s}=13 \mathrm{TeV}$

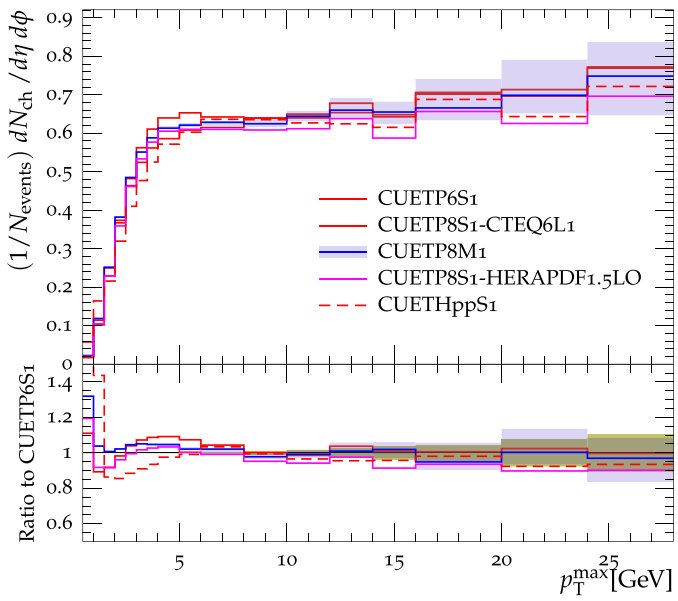

TransMAX charged-particle density $\sqrt{s}=13 \mathrm{TeV}$

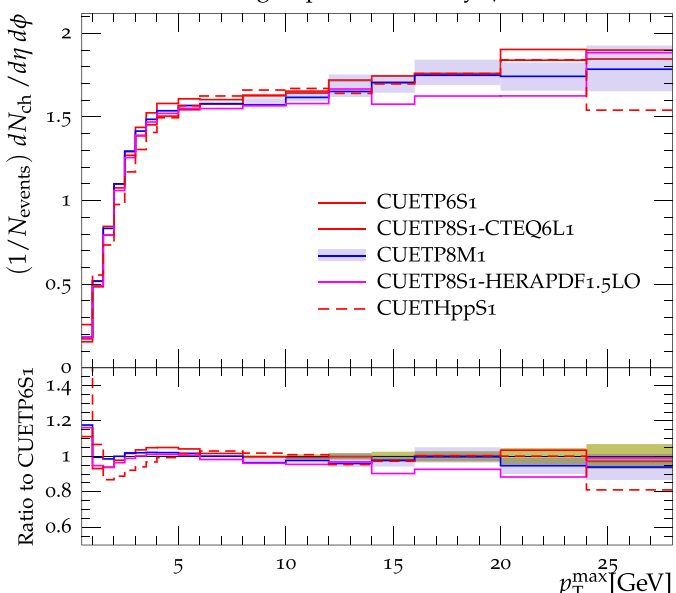

TransDIF charged-particle density $\sqrt{s}=13 \mathrm{TeV}$

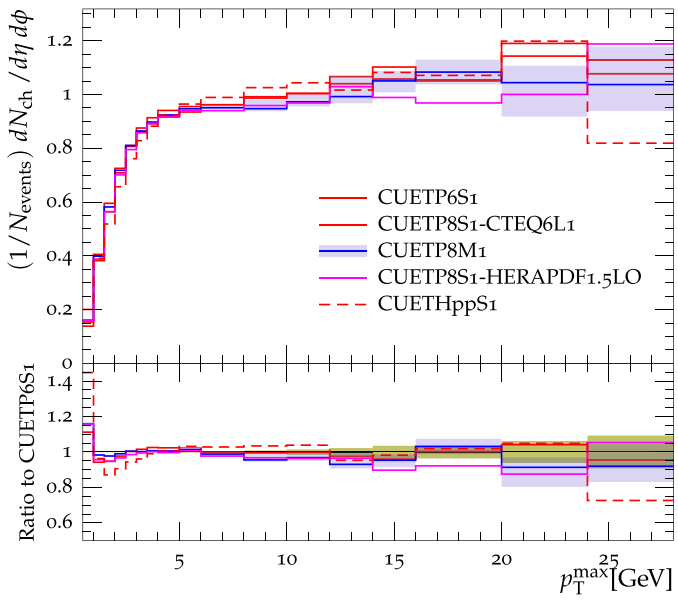

Fig. 19 Predictions at $\sqrt{s}=13 \mathrm{TeV}$ for the particle (left) and the $p_{\mathrm{T}}^{\text {sum }}$ densities (right) for charged particles with $p_{\mathrm{T}}>0.5 \mathrm{GeV}$ and $|\eta|<0.8$ in the TransMIN (top), TransMAX (middle), and TransDIF (bottom) regions, as defined by the leading charged particle, as a function of the leading charged-particle $p_{\mathrm{T}}^{\max }$ for the five CMS UE tunes: PYTHIA6 CUETP6S1-CTEQ6L1, and PYTHIA8 CUETP8S1-
TransMIN charged $p_{\mathrm{T}}^{\text {sum }}$ density $\sqrt{s}=13 \mathrm{TeV}$

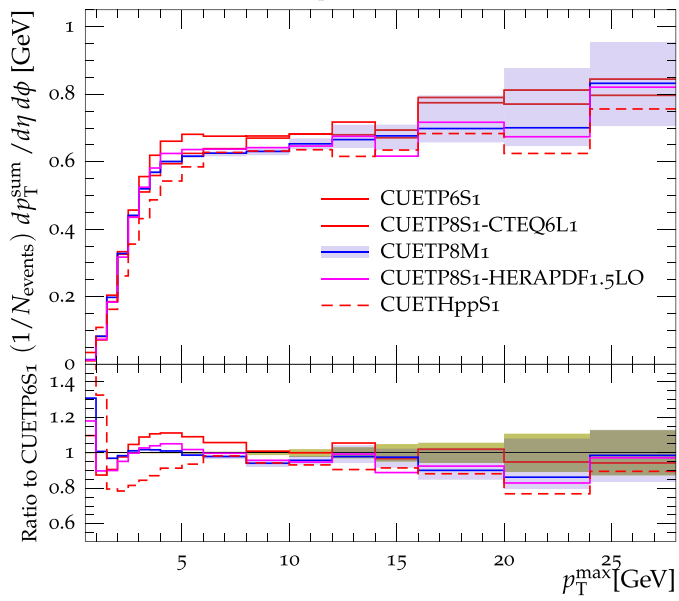

TransMAX charged $p_{\mathrm{T}}^{\text {sum }}$ density $\sqrt{s}=13 \mathrm{TeV}$
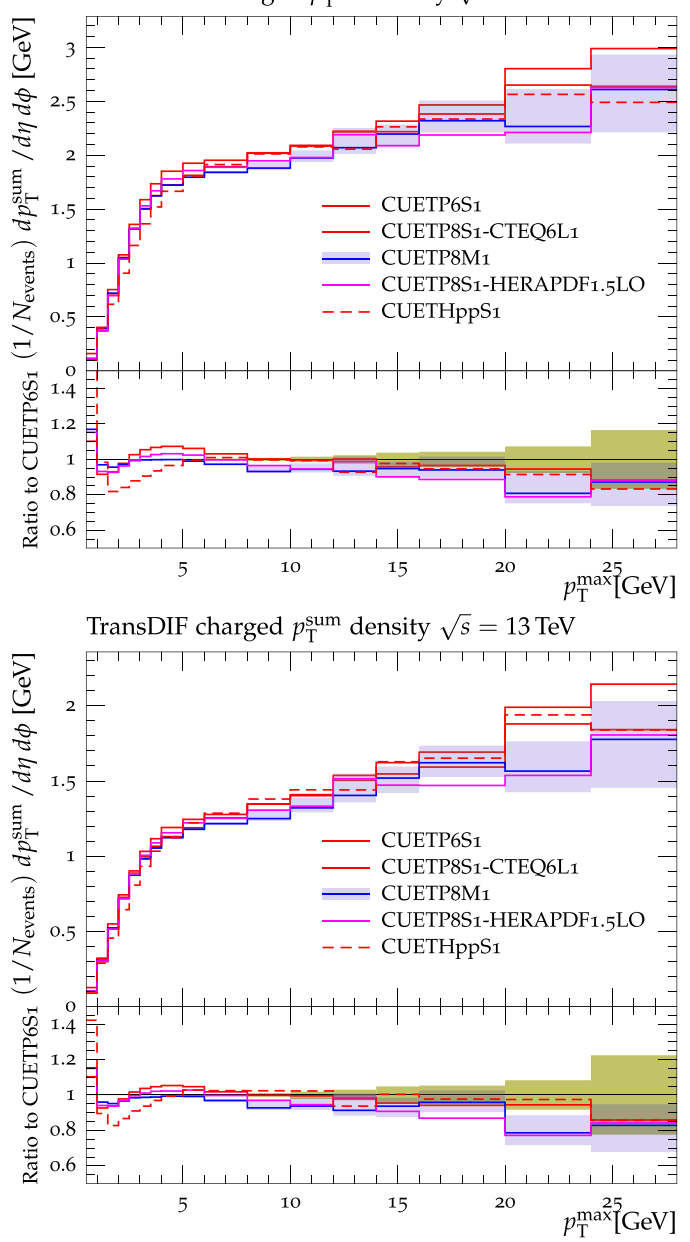

CTEQ6L1, CUETP8S1-HERAPDF1.5LO, and CUETP8M1, and HERWIG++ CUETHppS1. Also shown are the ratio of the tunes to predictions of CUETP8S1-CTEQ6L1. Predictions for CUETP8M1 are shown along with the envelope (green bands) of the corresponding eigentunes 

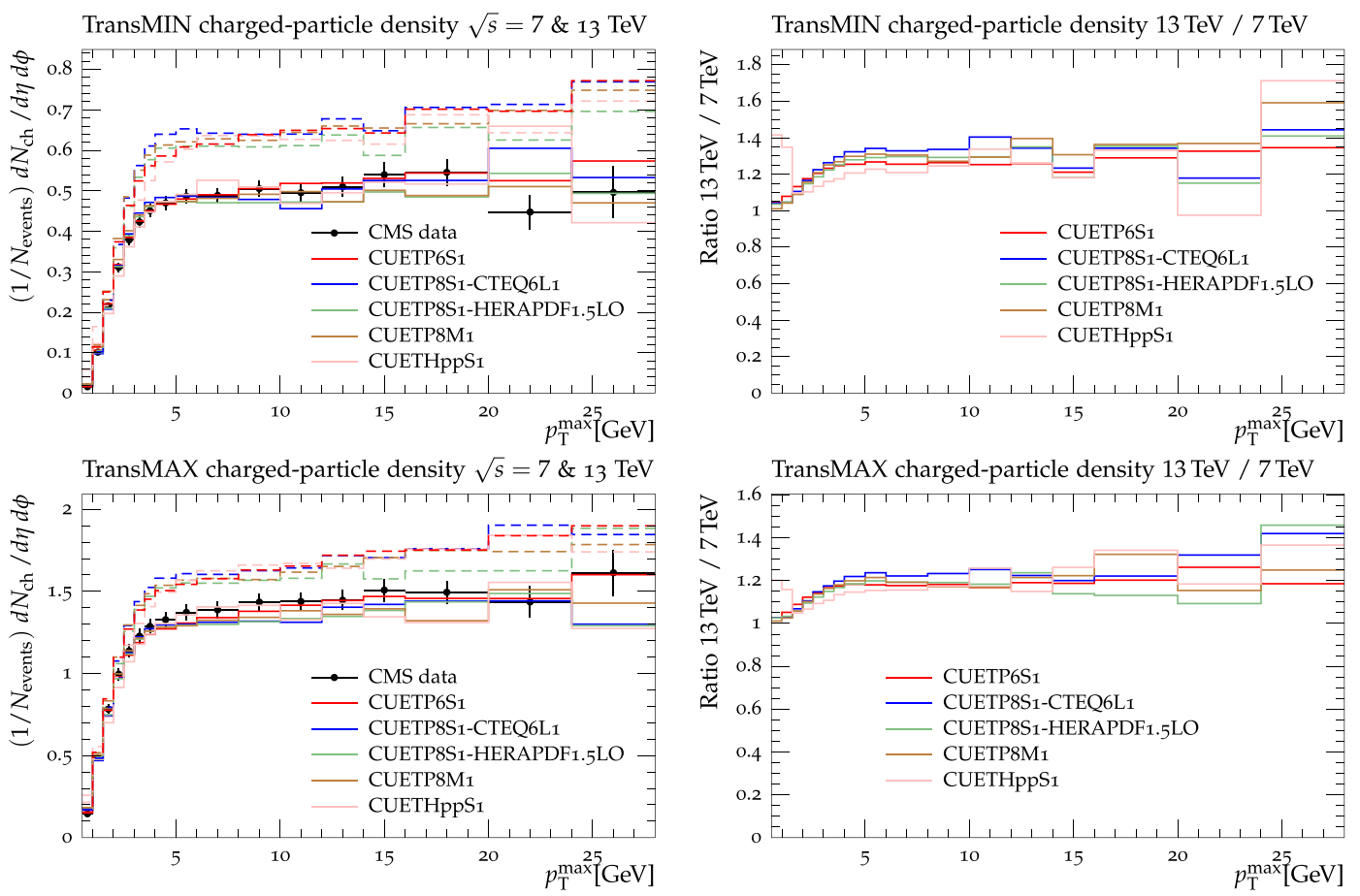

TransDIF charged-particle density $\sqrt{s}=7 \& 13 \mathrm{TeV}$

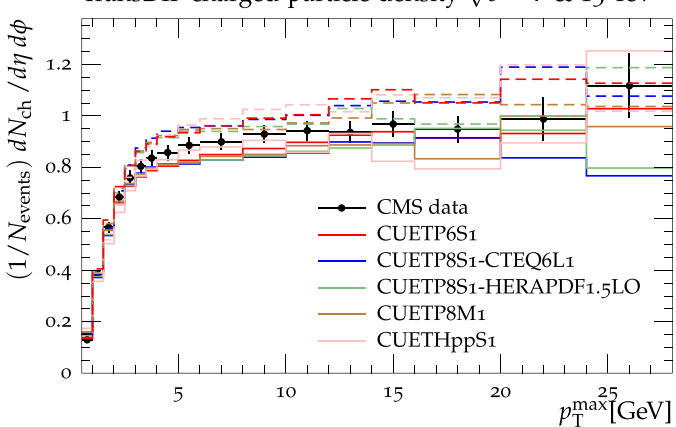

TransDIF charged-particle density $13 \mathrm{TeV} / 7 \mathrm{TeV}$

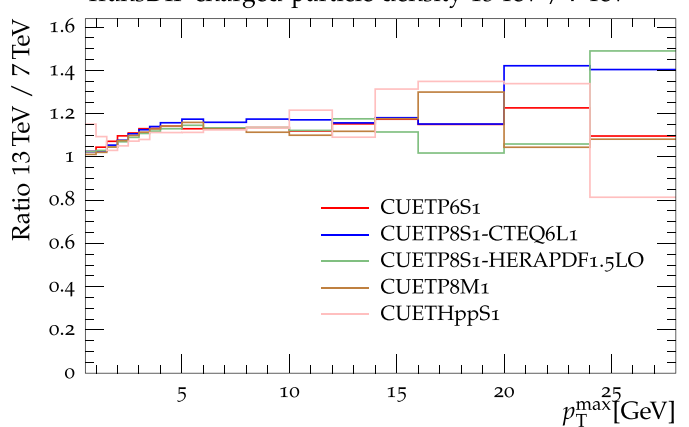

Fig. 20 Charged-particle density at $\sqrt{s}=7 \mathrm{TeV}$ for particles with $p_{\mathrm{T}}>0.5 \mathrm{GeV}$ and $|\eta|<0.8$ in the TransMIN (top), TransMAX (middle), and TransDIF (bottom) regions, as defined by the leading charged particle, as a function of the leading charged-particle $p_{\mathrm{T}}^{\max }$. The data are compared to PYTHIA6 using CUETP6S1-CTEQ6L1, to

PYTHIA8 using CUETP8S1-CTEQ6L1, CUETP8S1-HERAPDF1.5LO, and CUETP8M1, and to HERWIG++ using CUETHppS1. Also shown are the predictions (left) based on the CMS UE tunes at $13 \mathrm{TeV}$ (dashed lines), and the ratio of the $13 \mathrm{TeV}$ to $7 \mathrm{TeV}$ results for the five tunes (right)

UE tunes: CUETP6S1-CTEQ6L1, CUETP8S1-CTEQ6L1, CUETP8S1-HERAPDF1.5LO, CUETP8M1, and CUETHppS1. In Fig. 19 the ratio of the predictions using the four CMS tunes to the one using CUETP8M1 is shown. The predictions at $13 \mathrm{TeV}$ of all these tunes are remarkably similar. It does not seem to matter that the new CMS PYTHIA8 UE tunes do not fit very well to the $\sqrt{s}=300 \mathrm{GeV}$ UE data. The new PYTHIA 8 tunes give results at $13 \mathrm{TeV}$ similar to the new CMS PYTHIA6 tune and the new CMS HERWIG++ tune. The uncertainties on the predictions based on the eigentunes do not exceed $10 \%$ relative to the central value.

In Figs. 20 and 21 the predictions at $\sqrt{s}=13 \mathrm{TeV}$ obtained using the new tunes from $7 \mathrm{TeV}$ are shown for the charged-particle and the $p_{\mathrm{T}}^{\text {sum }}$ densities in the TransMIN,

TransMAX, and TransDIF regions, defined as a function of $p_{\mathrm{T}}^{\max }$. Also shown is the ratio of $13 \mathrm{TeV}$ to $7 \mathrm{TeV}$ results for the five tunes. The TransMIN region increases much more rapidly with energy than the TransDIF region. For example, when using CUETP8M1, the charged-particle and the $p_{\mathrm{T}}^{\text {sum }}$ densities in the TransMIN region for $5.0<p_{\mathrm{T}}^{\max }<6.0 \mathrm{GeV}$ is predicted to increase by 28 and $37 \%$, respectively, while the TransDIF region is predicted to increase by a factor of two less, i.e. by 13 and $18 \%$ respectively.

In Fig. 22, predictions obtained with PYTHIA8 using CUETP8S1-CTEQ6L1 and CUETP8M1, and Tune 4C are compared to the recent CMS data measured at $\sqrt{s}=$ $13 \mathrm{TeV}$ [53] on charged-particle multiplicity as a function of pseudorapidity. Predictions from CUETP8S1-CTEQ6L1 

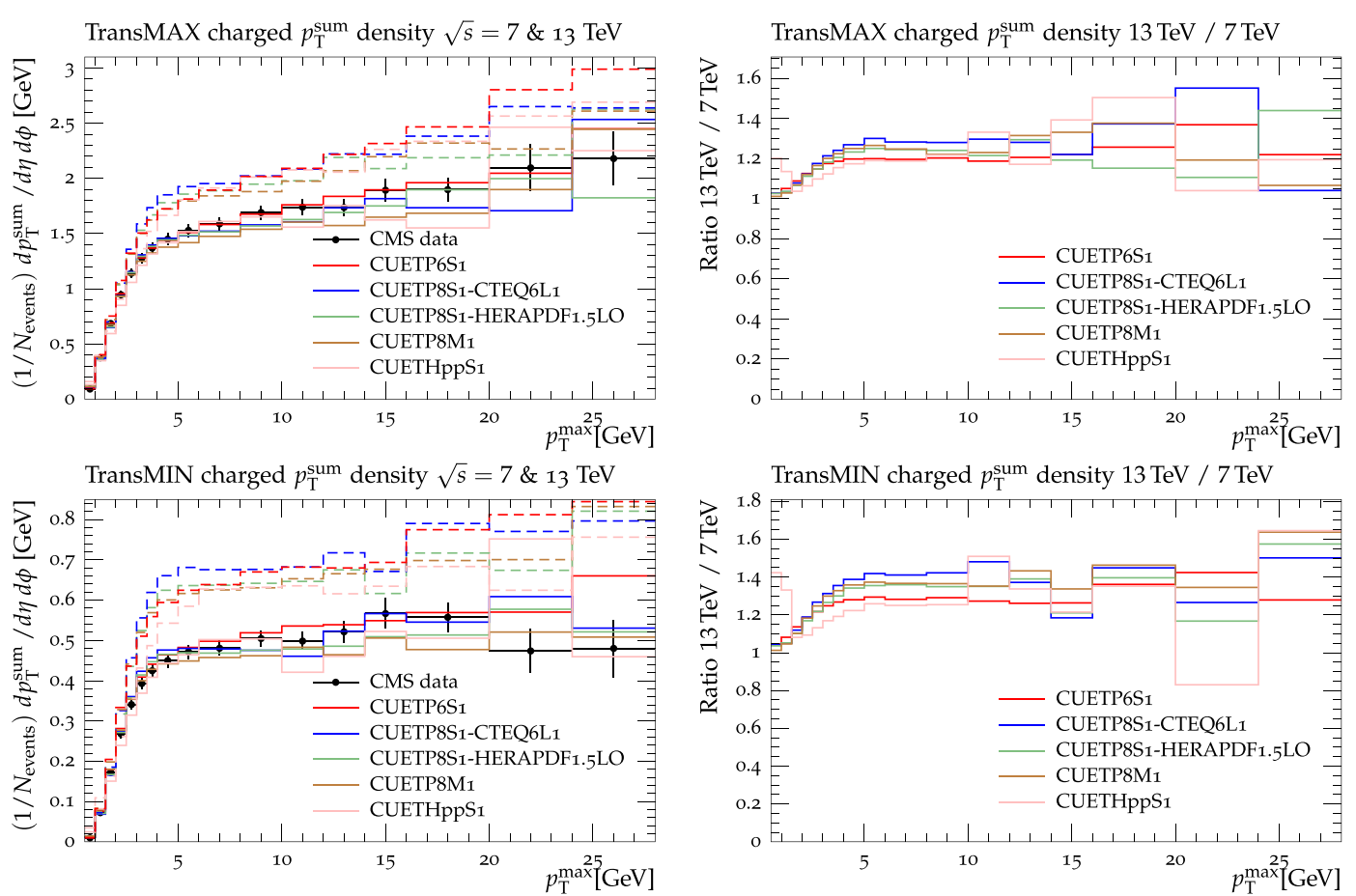

TransMIN charged $p_{\mathrm{T}}^{\text {sum }}$ density $13 \mathrm{TeV} / 7 \mathrm{TeV}$

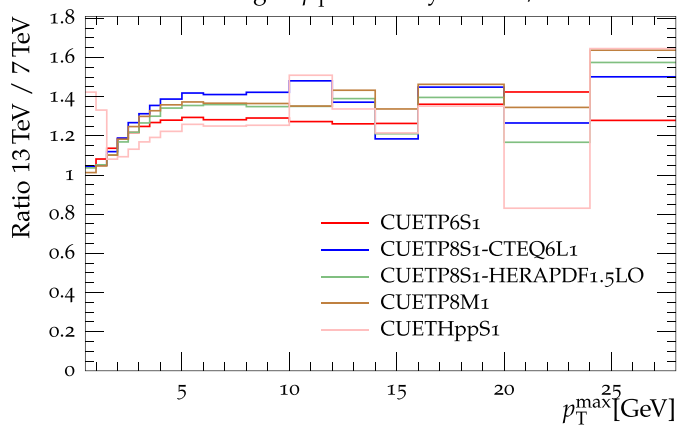

TransDIF charged $p_{\mathrm{T}}^{\text {sum }}$ density $13 \mathrm{TeV} / 7 \mathrm{TeV}$
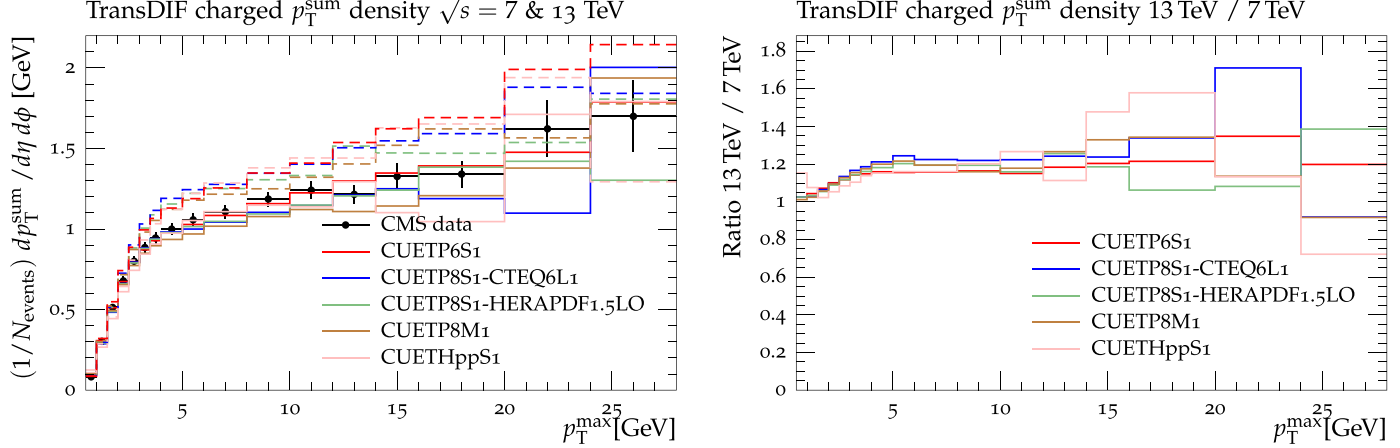

Fig. 21 Charged $p_{\mathrm{T}}^{\text {sum }}$ density at $\sqrt{s}=7 \mathrm{TeV}$ for particles with $p_{\mathrm{T}}>0.5 \mathrm{GeV}$ and $|\eta|<0.8$ in the TransMIN (top), TransMAX (middle), and TransDIF (bottom) regions, as defined by the leading charged particle, as a function of the leading charged-particle $p_{\mathrm{T}}^{\max }$. The data are compared to PYTHIA6 using CUETP6S1-CTEQ6L1, to

PYTHIA8 using CUETP8S1-CTEQ6L1, CUETP8S1-HERAPDF1.5LO, and CUETP8M1, and to HERWIG++ using CUETHppS1. Also shown are the predictions (left) based on the CMS UE tunes at $13 \mathrm{TeV}$ (dashed lines), and the ratio of the $13 \mathrm{TeV}$ to $7 \mathrm{TeV}$ results for the five tunes (right)

and CUETP8M1 are shown with the error bands corresponding to the uncertainties obtained from the eigentunes. These two new CMS tunes, although obtained from fits to UE data at $7 \mathrm{TeV}$, agree well with the MB measurements over the whole pseudorapidity range, while predictions from PYTHIA 8 Tune $4 \mathrm{C}$ overestimate the data by about $10 \%$. This confirms that the collision-energy dependence of the CMS UE tunes parameters can be trusted for predictions of MB observables.

\section{Summary and conclusions}

New tunes of the PYTHIA event generator were constructed for different parton distribution functions using various sets

of underlying-event (UE) data. By simultaneously fitting UE data at several center-of-mass energies, models for UE have been tested and their parameters constrained. The improvement in the description of UE data provided by the new CMS tunes at different collision energies gives confidence that they can provide reliable predictions at $\sqrt{s}=13 \mathrm{TeV}$, where all the new UE tunes predict similar results for the UE observables.

The observables sensitive to double-parton scattering (DPS) were fitted directly by tuning the MPI parameters. Two W+dijet DPS tunes and two four-jet DPS tunes were constructed to study the dependence of the DPS-sensitive observables on the MPI parameters. The CMS UE tunes perform fairly well in the description of DPS observables, but 


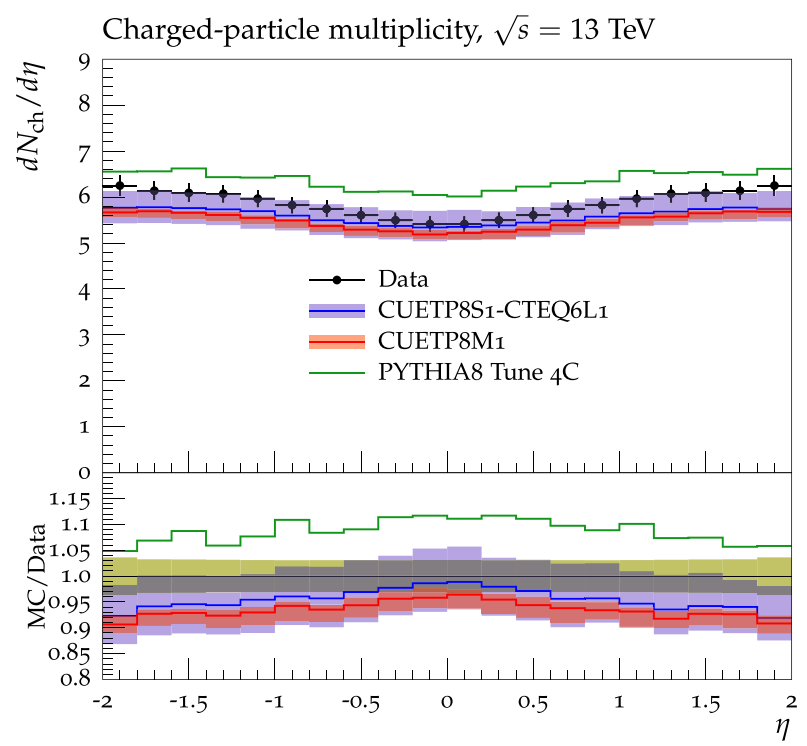

Fig. $22 \mathrm{CMS}$ data at $\sqrt{s}=13 \mathrm{TeV}$ [53] for the charged-particle pseudorapidity distribution, $\mathrm{dN}_{\mathrm{ch}} / \mathrm{d} \eta$, in inelastic proton-proton collisions. The data are compared to predictions of PYTHIA8 using CUETP8S1CTEQ6L1, CUETP8M1, and Tune 4C. The predictions based on CUETP8S1-CTEQ6L1 and CUETP8M1 are shown with an error band corresponding to the total uncertainty obtained from the eigentunes. Also shown are the ratios of these predictions to the data. The green band represents the total experimental uncertainty on the data

they do not fit the DPS data as well as the DPS tunes do. On the other hand, the CMS DPS tunes do not fit the UE data as well as the UE tunes. At present, it is not possible to accurately describe both soft and hard MPI within the current PYTHIA and HERWIG++ frameworks. Fitting DPS-sensitive observables has also provided the DPS effective cross section $\sigma_{\text {eff }}$ associated to each model. This method can be applied to determine the $\sigma_{\text {eff }}$ values associated with different MPI models implemented in the current MC event generators for the production of any final-state with two hard particles.

Predictions of PYTHIA8 using the CMS UE tunes agree fairly well with the MB observables in the central region $(|\eta|<2)$ and can be interfaced to higher-order and multileg matrix-element generators, such as POWHEG and MADGRAPH, while maintaining their good description of the UE. It is not necessary to produce separate tunes for these generators. In addition, we have verified that the measured particle pseudorapidity density at $13 \mathrm{TeV}$ is well reproduced by the new CMS UE Tunes. Furthermore, all of the new CMS tunes come with their eigentunes, which can be used to determine the uncertainties associated with the theoretical predictions. These new CMS tunes will play an important role in predicting and analyzing LHC data at 13 and $14 \mathrm{TeV}$.

Acknowledgments We congratulate our colleagues in the CERN accelerator departments for the excellent performance of the LHC and thank the technical and administrative staffs at CERN and at other CMS institutes for their contributions to the success of the CMS effort. In addition, we gratefully acknowledge the computing centres and personnel of the Worldwide LHC Computing Grid for delivering so effectively the computing infrastructure essential to our analyses. Finally, we acknowledge the enduring support for the construction and operation of the LHC and the CMS detector provided by the following funding agencies: the Austrian Federal Ministry of Science, Research and Economy and the Austrian Science Fund; the Belgian Fonds de la Recherche Scientifique, and Fonds voor Wetenschappelijk Onderzoek; the Brazilian Funding Agencies (CNPq, CAPES, FAPERJ, and FAPESP); the Bulgarian Ministry of Education and Science; CERN; the Chinese Academy of Sciences, Ministry of Science and Technology, and National Natural Science Foundation of China; the Colombian Funding Agency (COLCIENCIAS); the Croatian Ministry of Science, Education and Sport, and the Croatian Science Foundation; the Research Promotion Foundation, Cyprus; the Ministry of Education and Research, Estonian Research Council via IUT23-4 and IUT236 and European Regional Development Fund, Estonia; the Academy of Finland, Finnish Ministry of Education and Culture, and Helsinki Institute of Physics; the Institut National de Physique Nucléaire et de Physique des Particules/CNRS, and Commissariat à l'Énergie Atomique et aux Énergies Alternatives/CEA, France; the Bundesministerium für Bildung und Forschung, Deutsche Forschungsgemeinschaft, and Helmholtz-Gemeinschaft Deutscher Forschungszentren, Germany; the General Secretariat for Research and Technology, Greece; the National Scientific Research Foundation, and National Innovation Office, Hungary; the Department of Atomic Energy and the Department of Science and Technology, India; the Institute for Studies in Theoretical Physics and Mathematics, Iran; the Science Foundation, Ireland; the Istituto Nazionale di Fisica Nucleare, Italy; the Ministry of Science, ICT and Future Planning, and National Research Foundation (NRF), Republic of Korea; the Lithuanian Academy of Sciences; the Ministry of Education, and University of Malaya (Malaysia); the Mexican Funding Agencies (CINVESTAV, CONACYT, SEP, and UASLP-FAI); the Ministry of Business, Innovation and Employment, New Zealand; the Pakistan Atomic Energy Commission; the Ministry of Science and Higher Education and the National Science Centre, Poland; the Fundação para a Ciência e a Tecnologia, Portugal; JINR, Dubna; the Ministry of Education and Science of the Russian Federation, the Federal Agency of Atomic Energy of the Russian Federation, Russian Academy of Sciences, and the Russian Foundation for Basic Research; the Ministry of Education, Science and Technological Development of Serbia; the Secretaría de Estado de Investigación, Desarrollo e Innovación and Programa Consolider-Ingenio 2010, Spain; the Swiss Funding Agencies (ETH Board, ETH Zurich, PSI, SNF, UniZH, Canton Zurich, and SER); the Ministry of Science and Technology, Taipei; the Thailand Center of Excellence in Physics, the Institute for the Promotion of Teaching Science and Technology of Thailand, Special Task Force for Activating Research and the National Science and Technology Development Agency of Thailand; the Scientific and Technical Research Council of Turkey, and Turkish Atomic Energy Authority; the National Academy of Sciences of Ukraine, and State Fund for Fundamental Researches, Ukraine; the Science and Technology Facilities Council, UK; the US Department of Energy, and the US National Science Foundation. Individuals have received support from the Marie-Curie programme and the European Research Council and EPLANET (European Union); the Leventis Foundation; the A. P. Sloan Foundation; the Alexander von Humboldt Foundation; the Belgian Federal Science Policy Office; the Fonds pour la Formation à la Recherche dans l'Industrie et dans l'Agriculture (FRIA-Belgium); the Agentschap voor Innovatie door Wetenschap en Technologie (IWT-Belgium); the Ministry of Education, Youth and Sports (MEYS) of the Czech Republic; the Council of Science and Industrial Research, India; the HOMING PLUS programme of the Foundation for Polish Science, cofinanced from European Union, Regional Development Fund; the OPUS programme of the National Science Center (Poland); the Compagnia di San Paolo (Torino); MIUR project 20108T4XTM (Italy); the Thalis and Aris- 
teia programmes cofinanced by EU-ESF and the Greek NSRF; the National Priorities Research Program by Qatar National Research Fund; the Rachadapisek Sompot Fund for Postdoctoral Fellowship, Chulalongkorn University (Thailand); and the Welch Foundation, contract C-1845.

Open Access This article is distributed under the terms of the Creative Commons Attribution 4.0 International License (http://creativecomm ons.org/licenses/by/4.0/), which permits unrestricted use, distribution, and reproduction in any medium, provided you give appropriate credit to the original author(s) and the source, provide a link to the Creative Commons license, and indicate if changes were made.

Funded by SCOAP ${ }^{3}$.

\section{Appendix A: Tables of tune uncertainties}

This section provides the values of the parameters corresponding to the eigentunes of the new CMS PYTHIA8 and the HERWIG++ tunes. A change in the $\chi^{2}$ of the fit that equals the absolute $\chi^{2}$ value obtained in the tune defines the eigen- tunes listed in Tables 9, 10, 11 and 12 for the new PYTHIA8 and the new HERWIG++ tunes. The different parameter values indicated refer to the deviation tunes along each of the maximally independent directions in the parameter space, obtained by using the covariance matrix in the region of the best tune. The number of directions defined in the parameter space equals the number of free parameters $n$ used in the fit and results into $2 n$ parameter variations, i.e. eigentunes. These variations represent a good set of systematic errors on the given tune.

\section{Appendix B: Comparisons of PYTHIA6 UE tunes to data}

Figures 23, 24, 25 and 26 show the CDF data at $\sqrt{s}=0.3$, 0.9 , and $1.96 \mathrm{TeV}$, and the CMS data at $\sqrt{s}=7 \mathrm{TeV}$ on charged-particle and $p_{\mathrm{T}}^{\text {sum }}$ densities in the TransMIN and TransMAX regions, as a function of the transverse momen-

Table 9 Eigentunes sets for CUETP8S1-CTEQ6L1

\begin{tabular}{|c|c|c|c|c|c|c|c|c|}
\hline PYTHIA8 parameter & $1-$ & $1+$ & $2-$ & $2+$ & $3-$ & $3+$ & $4-$ & $4+$ \\
\hline MultipartonInteractions:pT0Ref [GeV] & 2.101 & 2.101 & 2.068 & 2.135 & 2.100 & 2.102 & 2.079 & 2.123 \\
\hline MultipartonInteractions:ecmPow & 0.191 & 0.231 & 0.210 & 0.211 & 0.231 & 0.191 & 0.191 & 0.231 \\
\hline MultipartonInteractions:expPow & 1.609 & 1.609 & 1.602 & 1.616 & 1.613 & 1.605 & 1.714 & 1.503 \\
\hline ColourReconnection:range & 3.030 & 3.609 & 3.313 & 3.313 & 3.311 & 3.314 & 3.314 & 3.311 \\
\hline
\end{tabular}

Table 10 Eigentunes sets for CUETP8S1-HERAPDF

\begin{tabular}{lllllllll}
\hline PYTHIA8 parameter & $1-$ & $1+$ & $2-$ & $2+$ & $3-$ & $3+$ & $4-$ & $4+$ \\
\hline MultipartonInteractions:pT0Ref $[\mathrm{GeV}]$ & 2.000 & 2.000 & 1.960 & 2.043 & 1.999 & 2.001 & 1.968 & 2.030 \\
MultipartonInteractions:ecmPow & 0.275 & 0.226 & 0.250 & 0.250 & 0.226 & 0.275 & 0.274 & 0.227 \\
MultipartonInteractions:expPow & 1.691 & 1.690 & 1.681 & 1.700 & 1.695 & 1.686 & 1.831 & 1.559 \\
ColourReconnection:range & 6.224 & 5.972 & 6.096 & 6.096 & 6.101 & 6.091 & 6.091 & 6.101 \\
\hline
\end{tabular}

Table 11 Eigentunes sets for CUETP8M1

\begin{tabular}{lllll}
\hline PYTHIA8 parameter & $1-$ & $1+$ & $2-$ & $2+$ \\
\hline MultipartonInteractions:pT0Ref [GeV] & 2.403 & 2.402 & 2.400 & 2.405 \\
MultipartonInteractions:ecmPow & 0.253 & 0.251 & 0.253 & 0.252 \\
\hline
\end{tabular}

Table 12 Eigentunes sets for CUETHppS1

\begin{tabular}{lllllllll}
\hline HERWIG++ parameter & $1-$ & $1+$ & $2-$ & $2+$ & $3-$ & $3+$ & $4-$ & $4+$ \\
\hline MPIHandler:InvRadius & 2.290 & 2.227 & 2.318 & 2.196 & 2.272 & 2.237 & 2.254 & 2.256 \\
RemnantDecayer:colourDisrupt & 0.396 & 0.811 & 0.634 & 0.623 & 0.632 & 0.625 & 0.596 & 0.666 \\
MPIHandler:Power & 0.396 & 0.351 & 0.331 & 0.408 & 0.399 & 0.342 & 0.361 & 0.381 \\
ColourReconnector:ReconnectionProbability & 0.615 & 0.460 & 0.529 & 0.527 & 0.523 & 0.533 & 0.444 & 0.626 \\
\hline
\end{tabular}




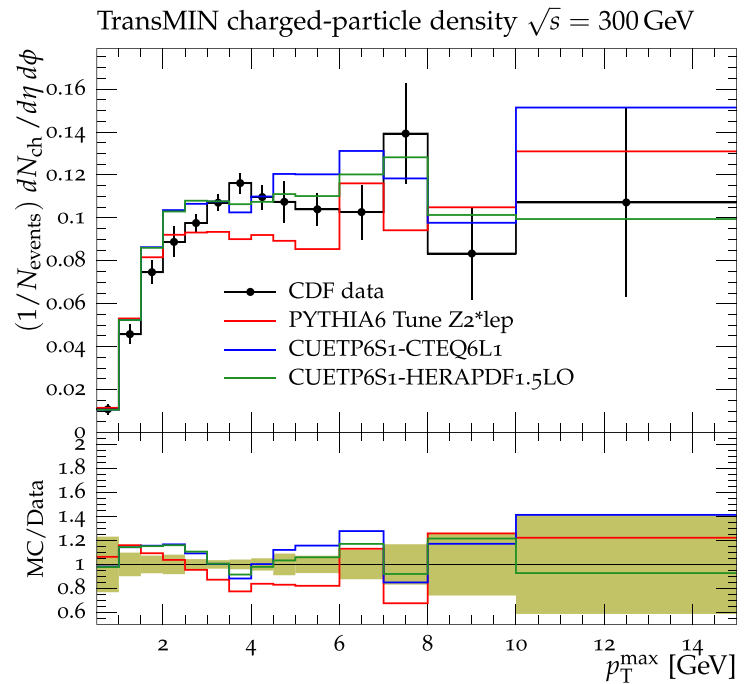

TransMIN charged $p_{\mathrm{T}}^{\text {sum }}$ density $\sqrt{s}=300 \mathrm{GeV}$

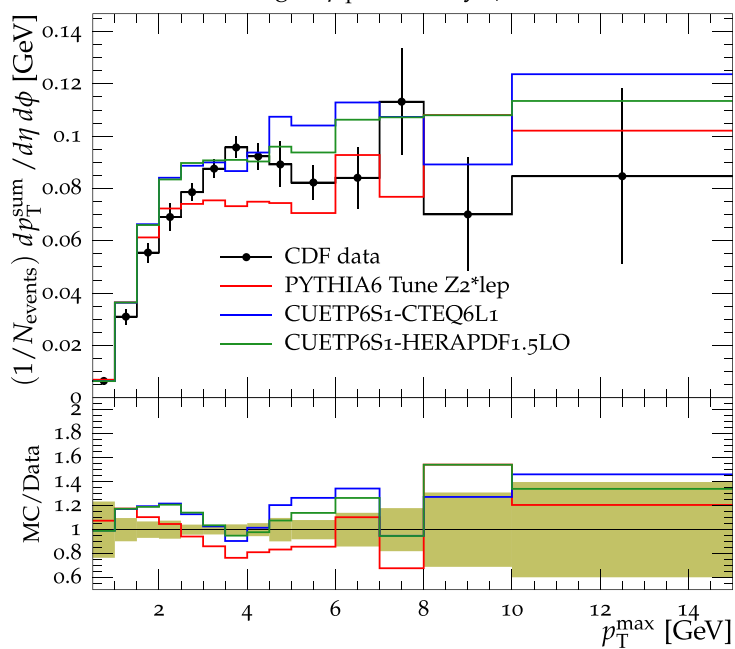

Fig. $23 \mathrm{CDF}$ data at $\sqrt{s}=300 \mathrm{GeV}$ [11] on the particle (top) and $p_{\mathrm{T}}^{\text {sum }}$ densities (bottom) for charged particles with $p_{\mathrm{T}}>0.5 \mathrm{GeV}$ and $|\eta|<0.8$ in the TransMIN (left) and TransMAX (right) regions as defined by the leading charged particle, as a function of the transverse

tum of the leading charged-particle $p_{\mathrm{T}}^{\max }$. The distributions are compared to predictions obtained with PYTHIA6 Tune Z2*lep and the two new CUETP6S1-CTEQ6L1 and CUETP6S1-HERAPDF1.5LO. The new CMS PYTHIA6 tunes are able to describe the measurements better than Tune $\mathrm{Z} 2 *$ lep, in both the rising and the plateau regions of the spectra.

\section{Appendix C: Comparisons to HERWIG++ UE tunes to data}

Figures 27, 28, 29 and 30 show the CDF data at $\sqrt{s}=0.3$, 0.9 , and $1.96 \mathrm{TeV}$, and the CMS data at $\sqrt{s}=7 \mathrm{TeV}$ on
TransMAX charged-particle density $\sqrt{s}=300 \mathrm{GeV}$

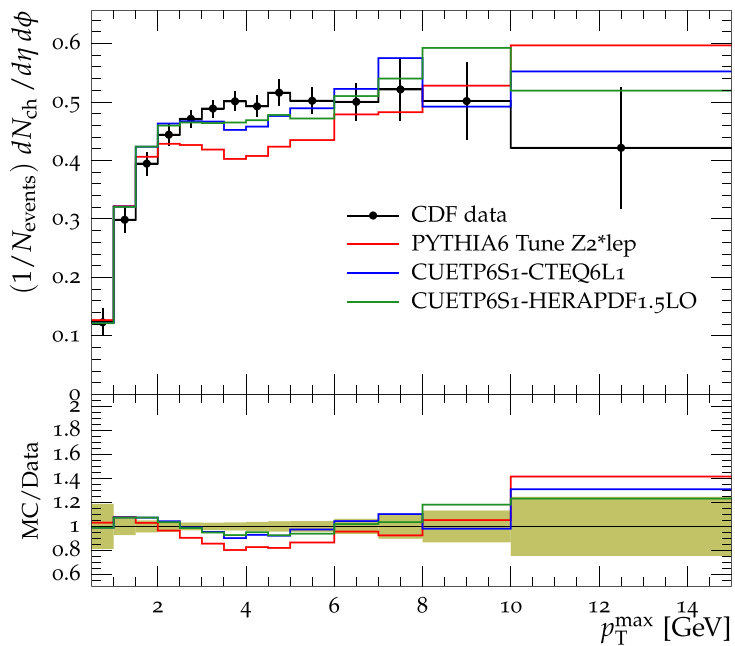

TransMAX charged $p_{\mathrm{T}}^{\text {sum }}$ density $\sqrt{s}=300 \mathrm{GeV}$

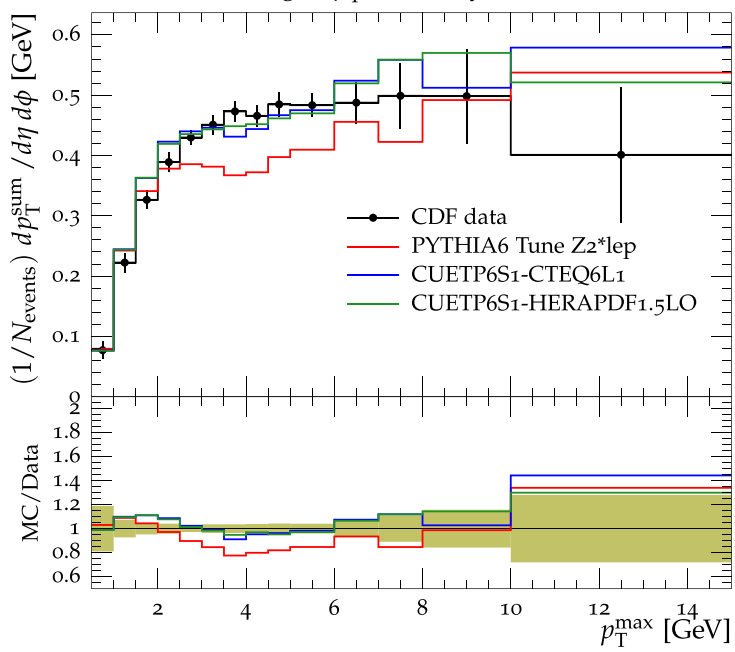

momentum of the leading charged-particle $p_{\mathrm{T}}^{\max }$. The data are compared to the PYTHIA6 Tune Z2*lep, CUETP6S1-CTEQ6L1 and CUETP6S1HERAPDF1.5LO. The green bands in the ratios represent the total experimental uncertainties

the charged-particle and $p_{\mathrm{T}}^{\mathrm{sum}}$ densities in the TransMIN and TransMAX regions as a function of $p_{\mathrm{T}}^{\max }$, and compared with predictions obtained with the HERWIG++ Tune UE-EE-5C and the new CUETHppS1. These two HERWIG++ tunes are very similar and adequately describe the UE data at all four energies.

\section{Appendix D: Additional comparisons at $13 \mathrm{TeV}$}

In this section, a supplementary collection of comparisons among predictions of the new tunes are shown for DPS and $\mathrm{MB}$ observables at $13 \mathrm{TeV}$. 

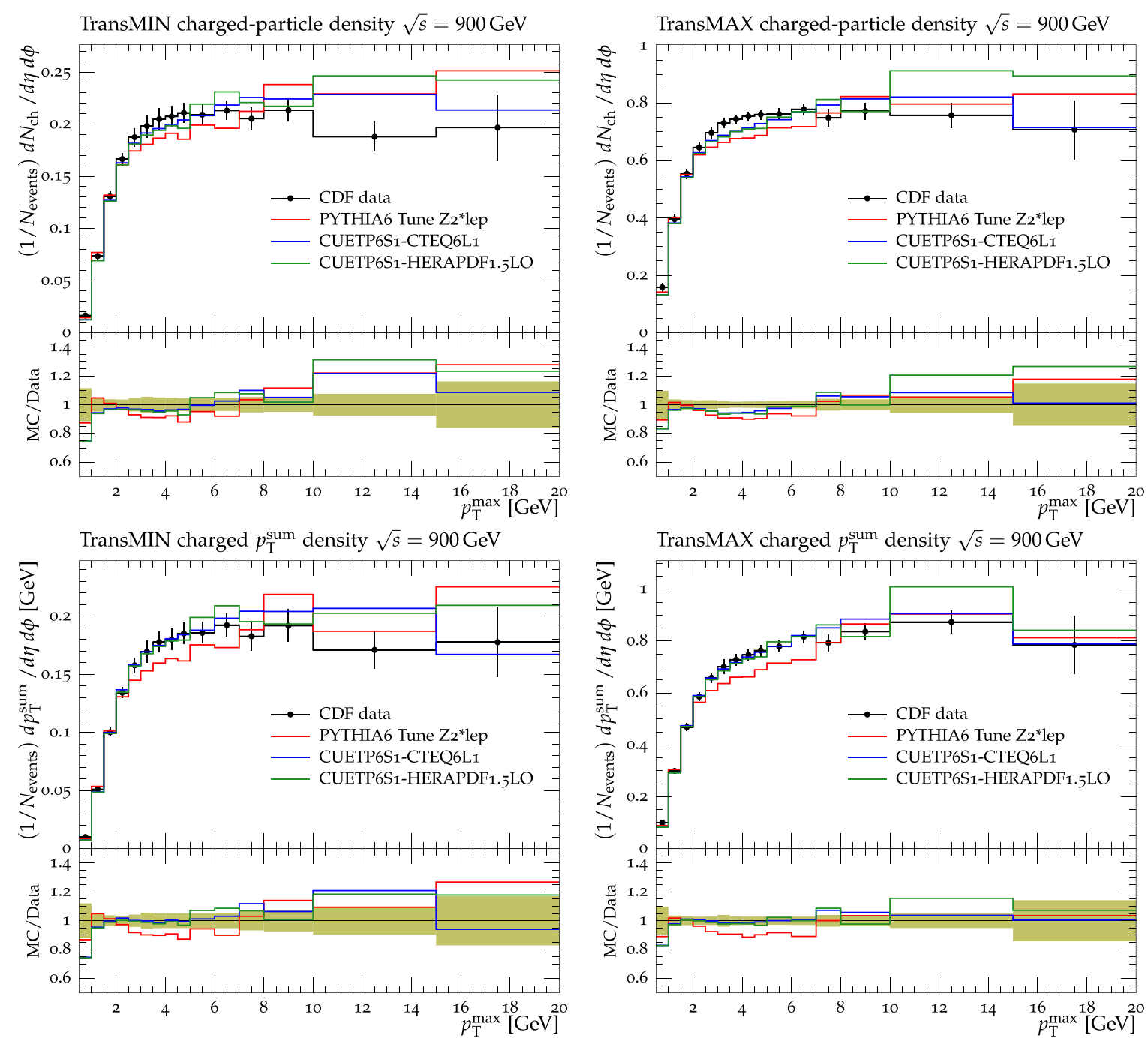

Fig. $24 \mathrm{CDF}$ data at $\sqrt{s}=900 \mathrm{GeV}$ [11] on the particle (top) and $p_{\mathrm{T}}^{\text {sum }}$ densities (bottom) for charged particles with $p_{\mathrm{T}}>0.5 \mathrm{GeV}$ and $|\eta|<0.8$ in the TransMIN (left) and TransMAX (right) regions as defined by the leading charged particle, as a function of the transverse

momentum of the leading-charged particle $p_{\mathrm{T}}^{\max }$. The data are compared to the PYTHIA6 Tune Z2*lep, CUETP6S1-CTEQ6L1 and CUETP6S1HERAPDF1.5LO. The green bands in the ratios represent the total experimental uncertainties

\section{D.1 DPS predictions at $13 \mathrm{TeV}$}

In Fig. 31, the predictions for the DPS-sensitive observables at $13 \mathrm{TeV}$ are shown for the three CMS PYTHIA8 UE tunes: CUETP8S1-CTEQ6L1, CUETP8S1-HERAPDF1.5LO, and CUETP8M1, for CUETHppS1, and for the two CMS PYTHIA8 DPS tunes CDPSTP8S1-4j and CDPSTP8S2-4j. In HERWIG++, $\sigma_{\text {eff }}$ is independent of the center-of-mass energy, while PYTHIA8 gives a $\sigma_{\text {eff }}$ that increases with energy. The PYTHIA8 UE tunes predict that $\sigma_{\text {eff }}$ will increase by about $7 \%$ between 7 and $13 \mathrm{TeV}$, while the CDPSTP8S2-4j predicts an increase of about $20 \%$. This results in slightly different predictions for the DPS-sensitive observables at $13 \mathrm{TeV}$ for the CMS UE tunes and the CMS DPS tunes.

\section{D.2 MB predictions at $13 \mathrm{TeV}$}

Predictions of the CMS UE tunes at $\sqrt{s}=13 \mathrm{TeV}$ are shown in Fig. 32 for the charged-particle pseudorapidity distribution, $\mathrm{dN}_{\mathrm{ch}} / \mathrm{d} \eta$, for inelastic, non single-diffraction-enhanced, and single-diffraction-enhanced proton-proton collisions. In Fig. 32, the ratio of 13 to $8 \mathrm{TeV}$ results is shown for each of the tunes. The densities in the forward region are predicted to increase more rapidly than the central region between 8 and $13 \mathrm{TeV}$. However, the UE observables in Figs. 20 and 21 increase much faster with center-of-mass energy than do these MB observables. 

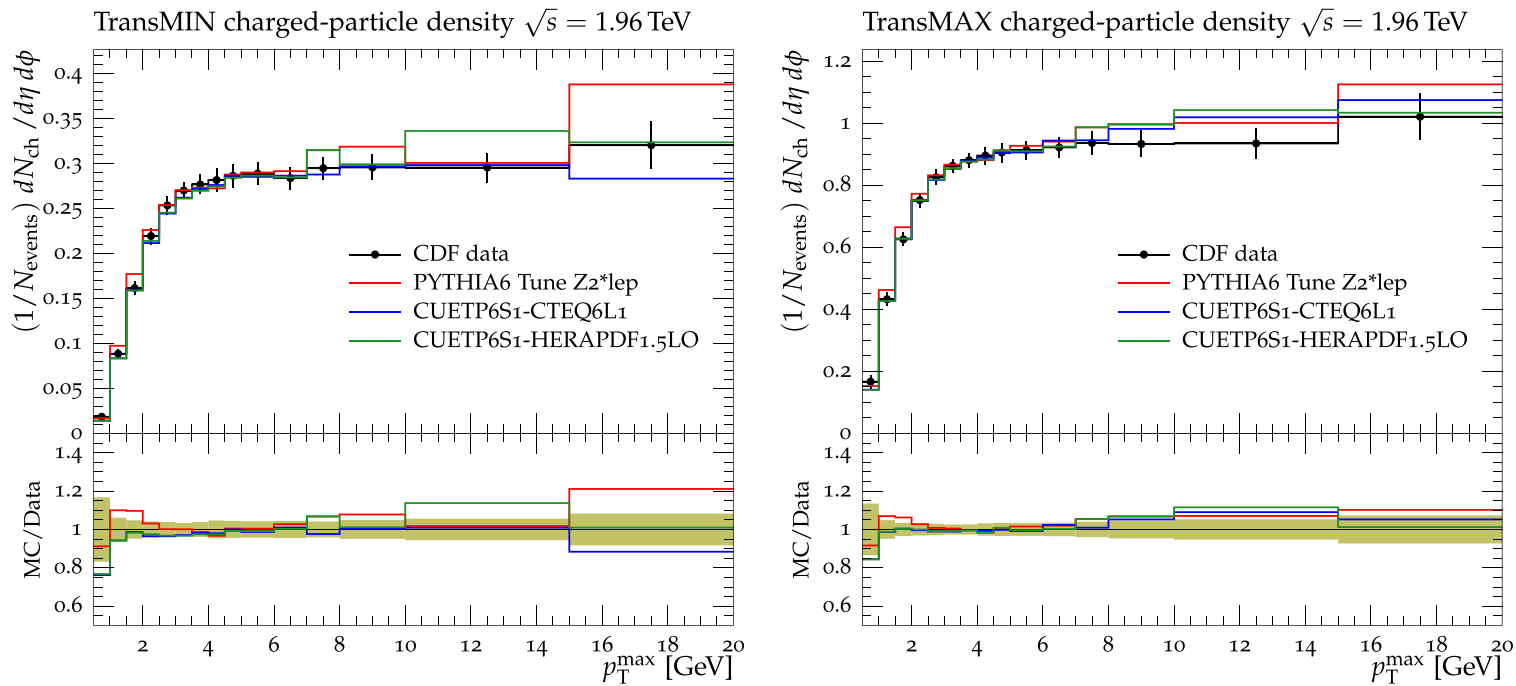

TransMIN charged $p_{\mathrm{T}}^{\text {sum }}$ density $\sqrt{s}=1.96 \mathrm{TeV}$

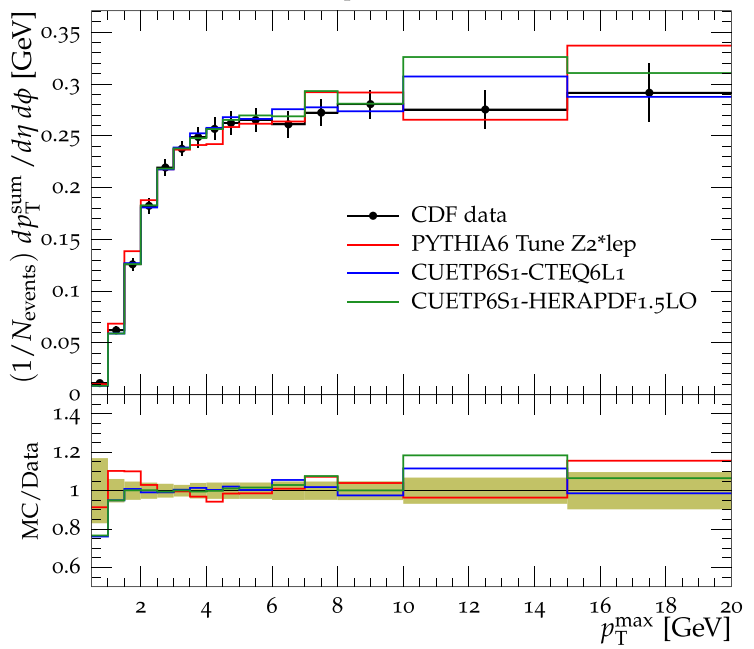

TransMAX charged $p_{\mathrm{T}}^{\text {sum }}$ density $\sqrt{s}=1.96 \mathrm{TeV}$

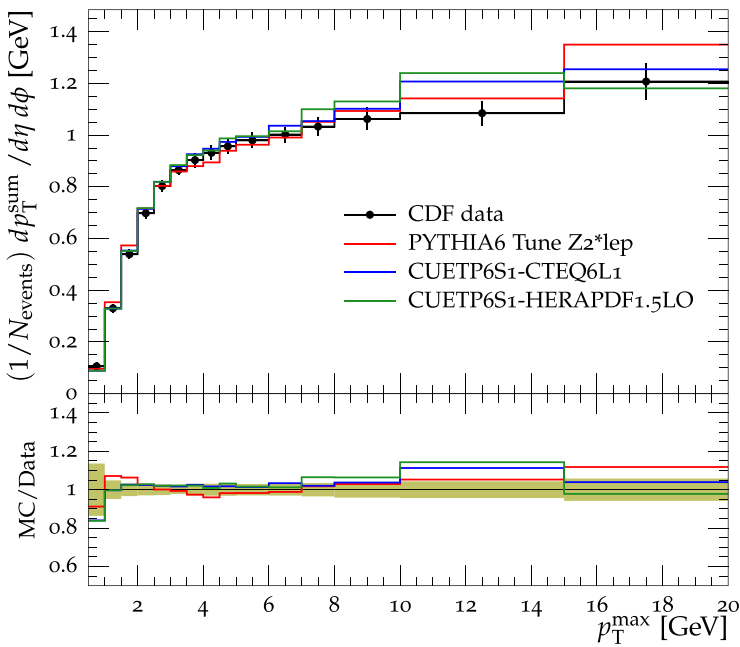

Fig. $25 \mathrm{CDF}$ data at $\sqrt{s}=1.96 \mathrm{TeV}$ [11] on the particle (top) and $p_{\mathrm{T}}^{\text {sum }}$ densities (bottom) for charged particles with $p_{\mathrm{T}}>0.5 \mathrm{GeV}$ and $|\eta|<0.8$ in the TransMIN (left) and TransMAX (right) regions as defined by the leading charged particle, as a function of the transverse

momentum of the leading charged-particle $p_{\mathrm{T}}^{\max }$. The data are compared to the PYTHIA6 Tune Z2*lep, CUETP6S1-CTEQ6L1 and CUETP6S1HERAPDF1.5LO. The green bands in the ratios represent the total experimental uncertainties 
TransMIN charged-particle density $\sqrt{s}=7 \mathrm{TeV}$

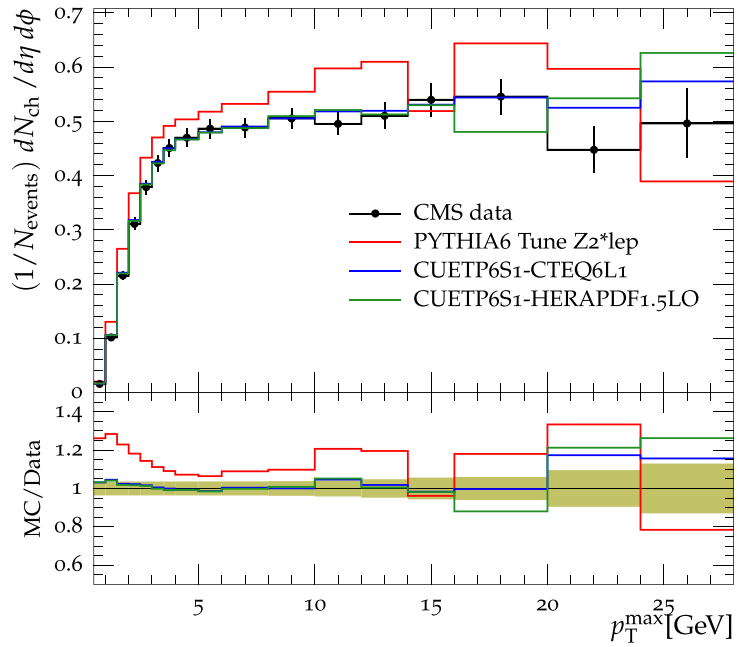

TransMIN charged $p_{\mathrm{T}}^{\text {sum }}$ density $\sqrt{s}=7 \mathrm{TeV}$

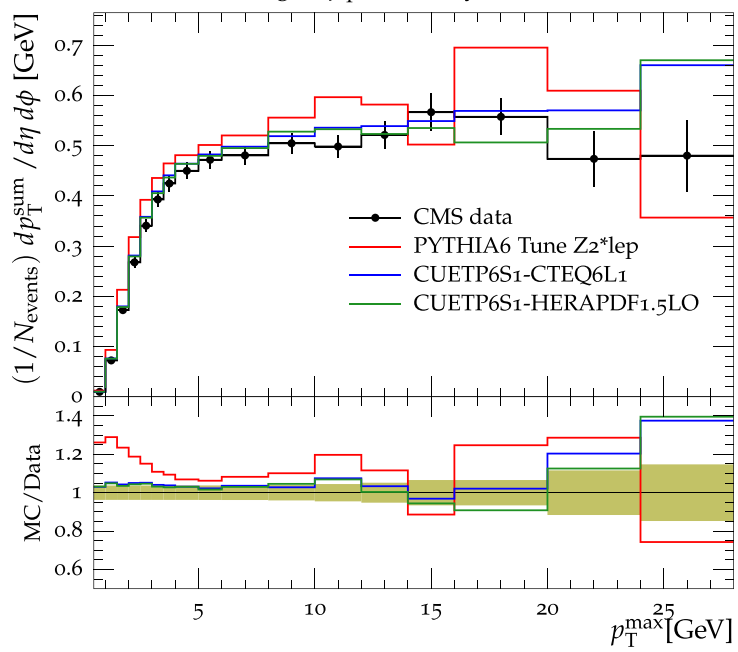

Fig. 26 CMS data at $\sqrt{s}=7 \mathrm{TeV}$ [17] on the particle (top) and $p_{\mathrm{T}}^{\text {sum }}$ densities (bottom) for charged particles with $p_{\mathrm{T}}>0.5 \mathrm{GeV}$ and $|\eta|<0.8$ in the TransMIN (left) and TransMAX (right) regions as defined by the leading charged particle, as a function of the transverse momen-
TransMAX charged-particle density $\sqrt{s}=7 \mathrm{TeV}$

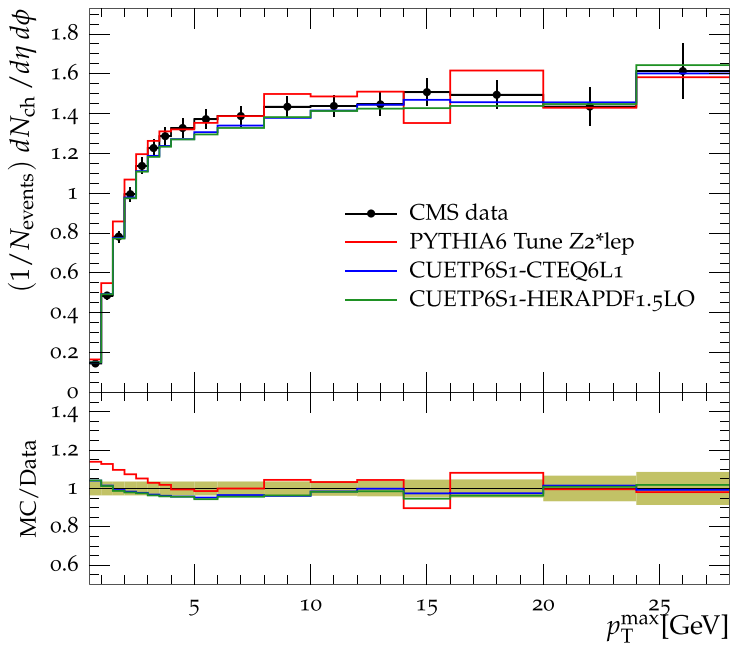

TransMAX charged $p_{\mathrm{T}}^{\text {sum }}$ density $\sqrt{s}=7 \mathrm{TeV}$

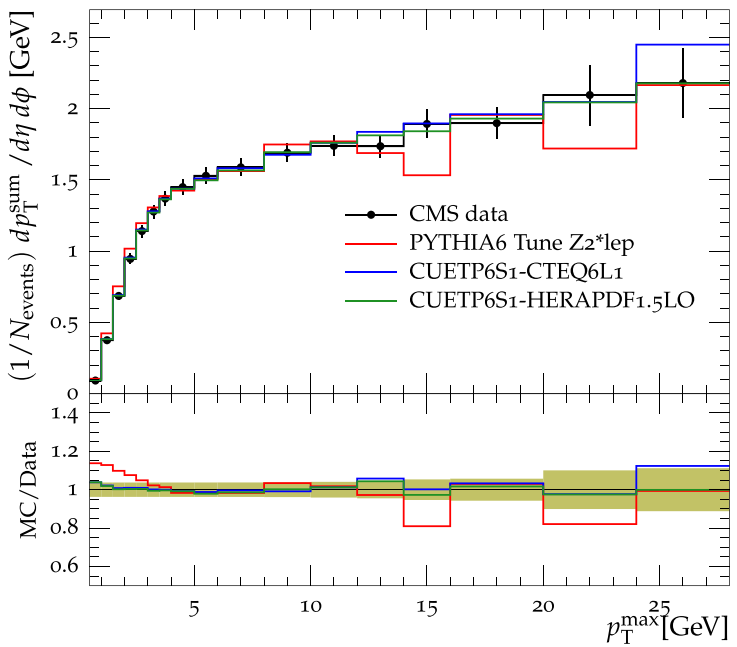

tum of the leading charged-particle $p_{\mathrm{T}}^{\max }$. The data are compared to the PYTHIA6 Tune Z2*lep, CUETP6S1-CTEQ6L1 and CUETP6S1HERAPDF1.5LO. The green bands in the ratios represent the total experimental uncertainties 

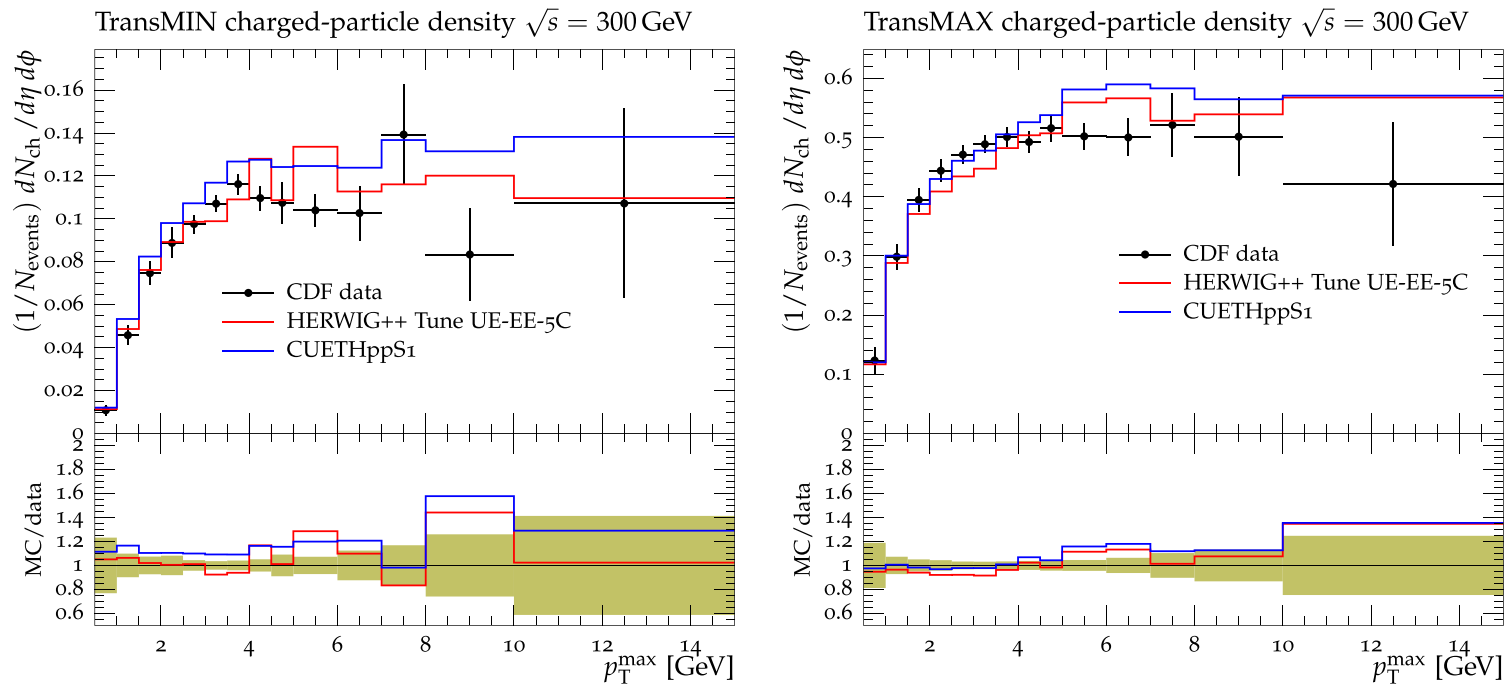

TransMIN charged $p_{\mathrm{T}}^{\text {sum }}$ density $\sqrt{ } s=300 \mathrm{GeV}$
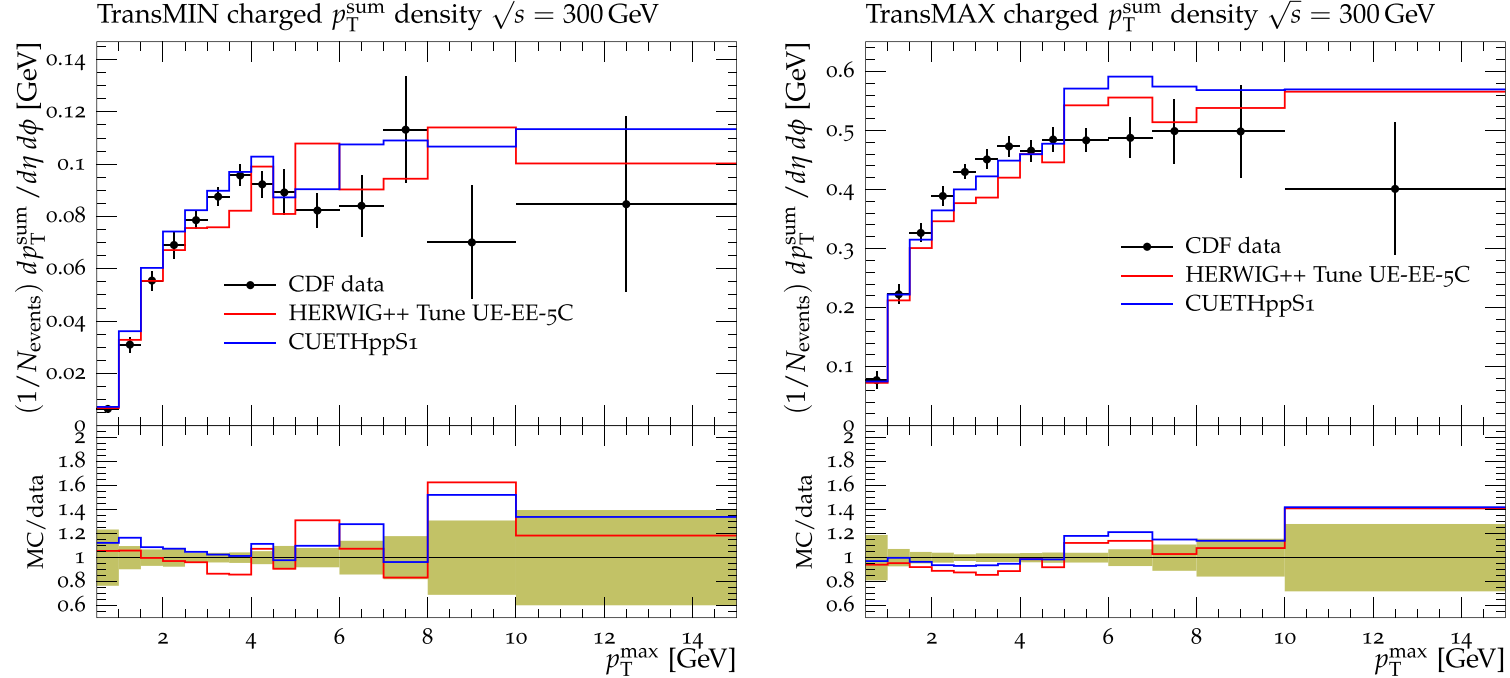

Fig. $27 \mathrm{CDF}$ data at $\sqrt{s}=300 \mathrm{GeV}$ [11] on particle (top) and $p_{\mathrm{T}}^{\text {sum }}$ densities (bottom) for charged particles with $p_{\mathrm{T}}>0.5 \mathrm{GeV}$ and $|\eta|<0.8$ in the TransMIN (left) and TransMAX (right) regions as defined by the leading charged particle, as a function of the transverse momentum of

the leading charged-particle $p_{\mathrm{T}}^{\max }$. The data are compared to the HERWIG++ Tune UE-EE-5C and CUETHppS1. The green bands in the ratios represent the total experimental uncertainties 

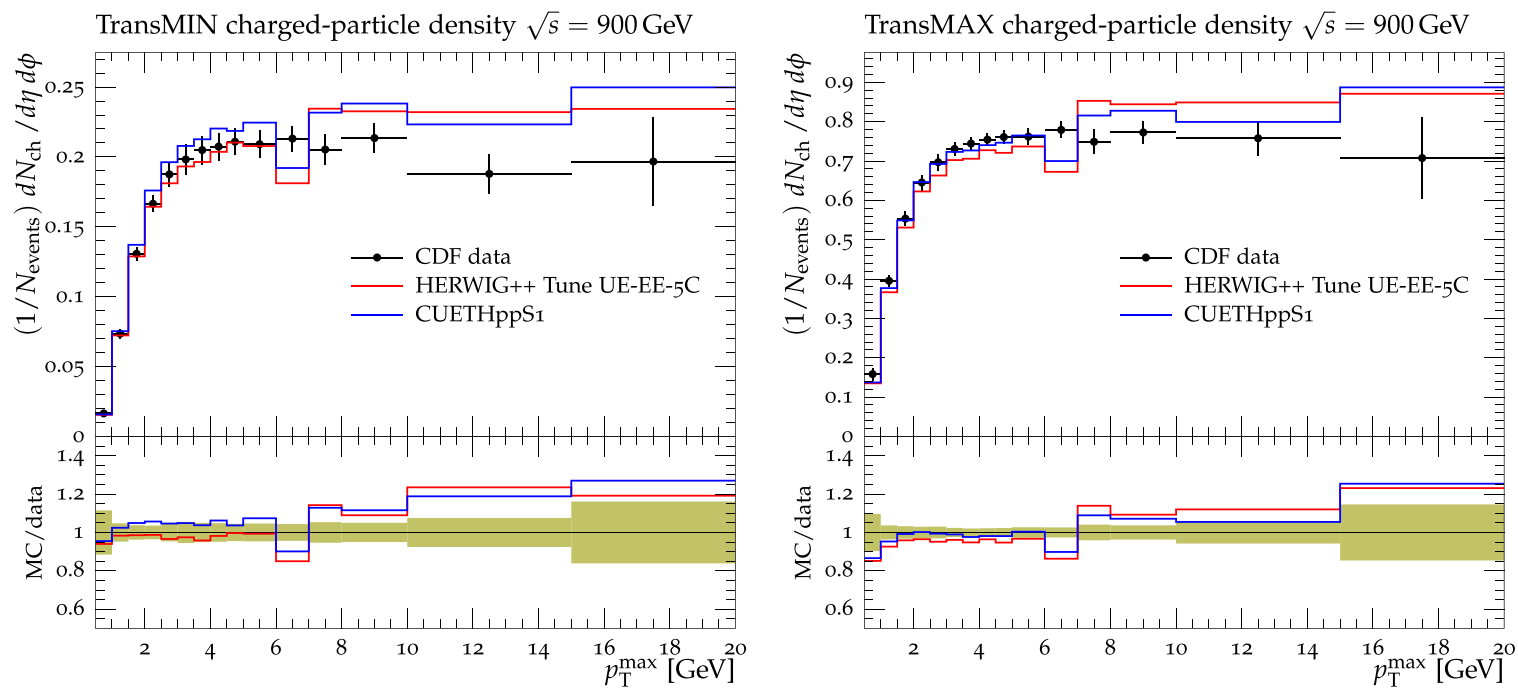

TransMIN charged $p_{\mathrm{T}}^{\text {sum }}$ density $\sqrt{s}=900 \mathrm{GeV}$

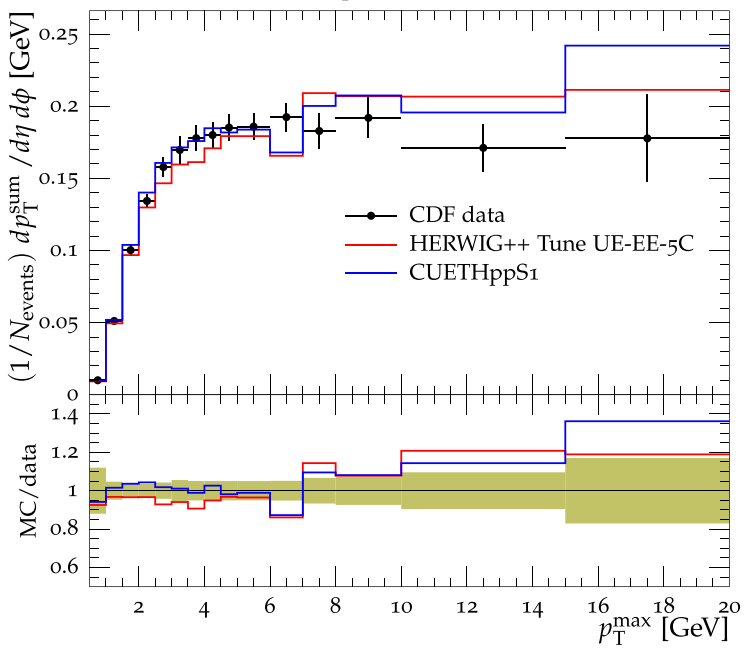

TransMAX charged $p_{\mathrm{T}}^{\text {sum }}$ density $\sqrt{s}=900 \mathrm{GeV}$

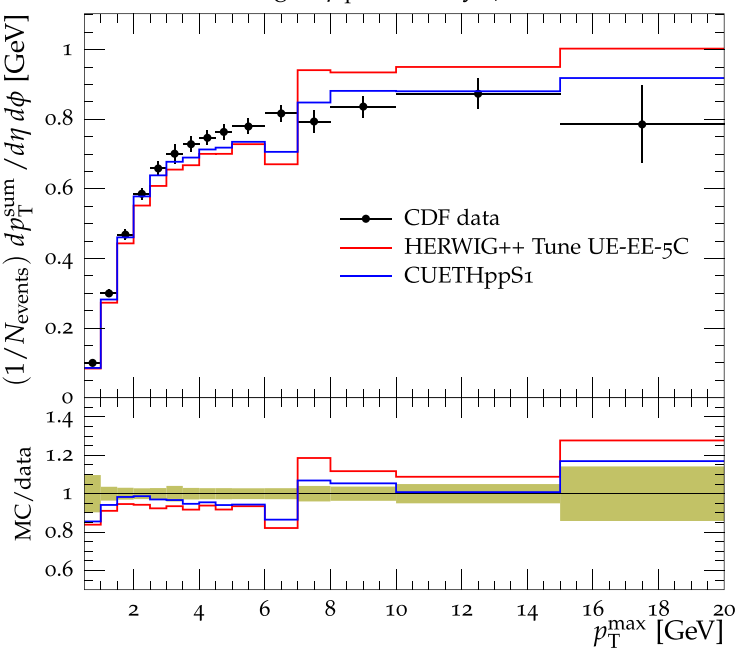

Fig. $28 \mathrm{CDF}$ data at $\sqrt{s}=900 \mathrm{GeV}$ [11] on particle (top) and $p_{\mathrm{T}}^{\text {sum }}$ densities (bottom) for charged particles with $p_{\mathrm{T}}>0.5 \mathrm{GeV}$ and $|\eta|<0.8$ in the TransMIN (left) and TransMAX (right) regions as defined by the leading charged particle, as a function of the transverse momentum of

the leading charged-particle $p_{\mathrm{T}}^{\max }$. The data are compared to the HERWIG++ Tune UE-EE-5C and CUETHppS1. The green bands in the ratios represent the total experimental uncertainties 

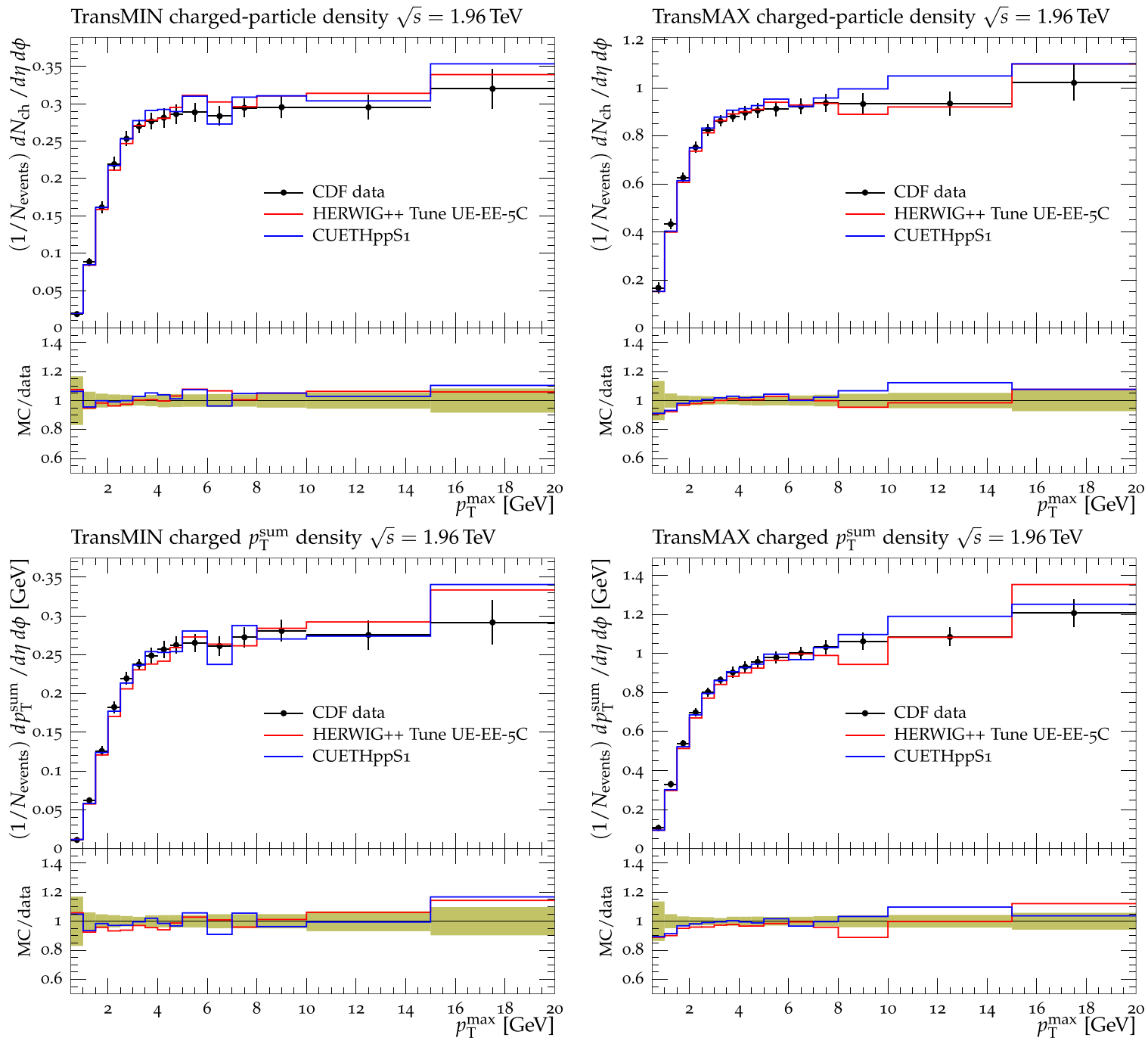

Fig. $29 \mathrm{CDF}$ data at $\sqrt{s}=1.96 \mathrm{TeV}$ [11] on particle (top) and $p_{\mathrm{T}}^{\text {sum }}$ densities (bottom) for charged particles with $p_{\mathrm{T}}>0.5 \mathrm{GeV}$ and $|\eta|<0.8$ in the TransMIN (left) and TransMAX (right) regions as defined by the leading charged particle, as a function of the transverse momentum of

the leading charged-particle $p_{\mathrm{T}}^{\max }$. The data are compared to the HERWIG++ Tune UE-EE-5C and CUETHppS1. The green bands in the ratios represent the total experimental uncertainties 

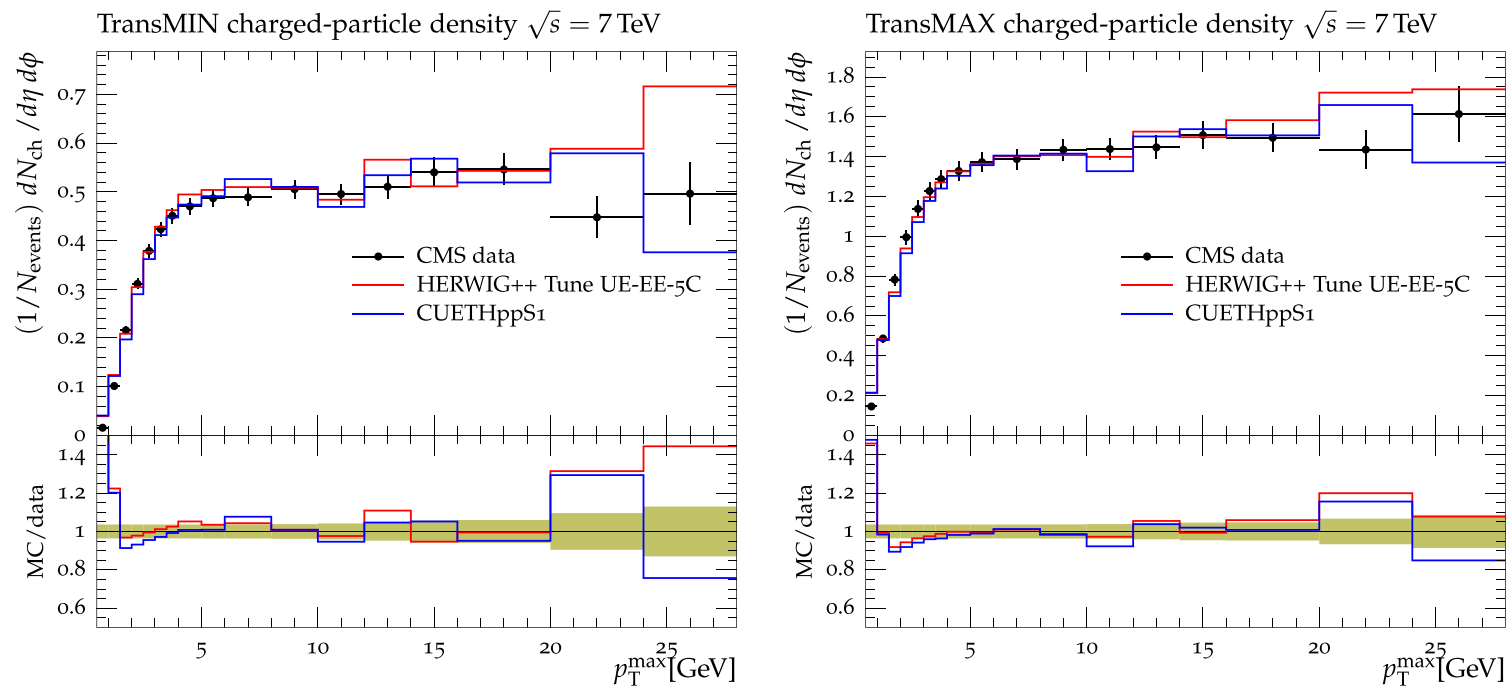

TransMIN charged $p_{\mathrm{T}}^{\text {sum }}$ density $\sqrt{s}=7 \mathrm{TeV}$
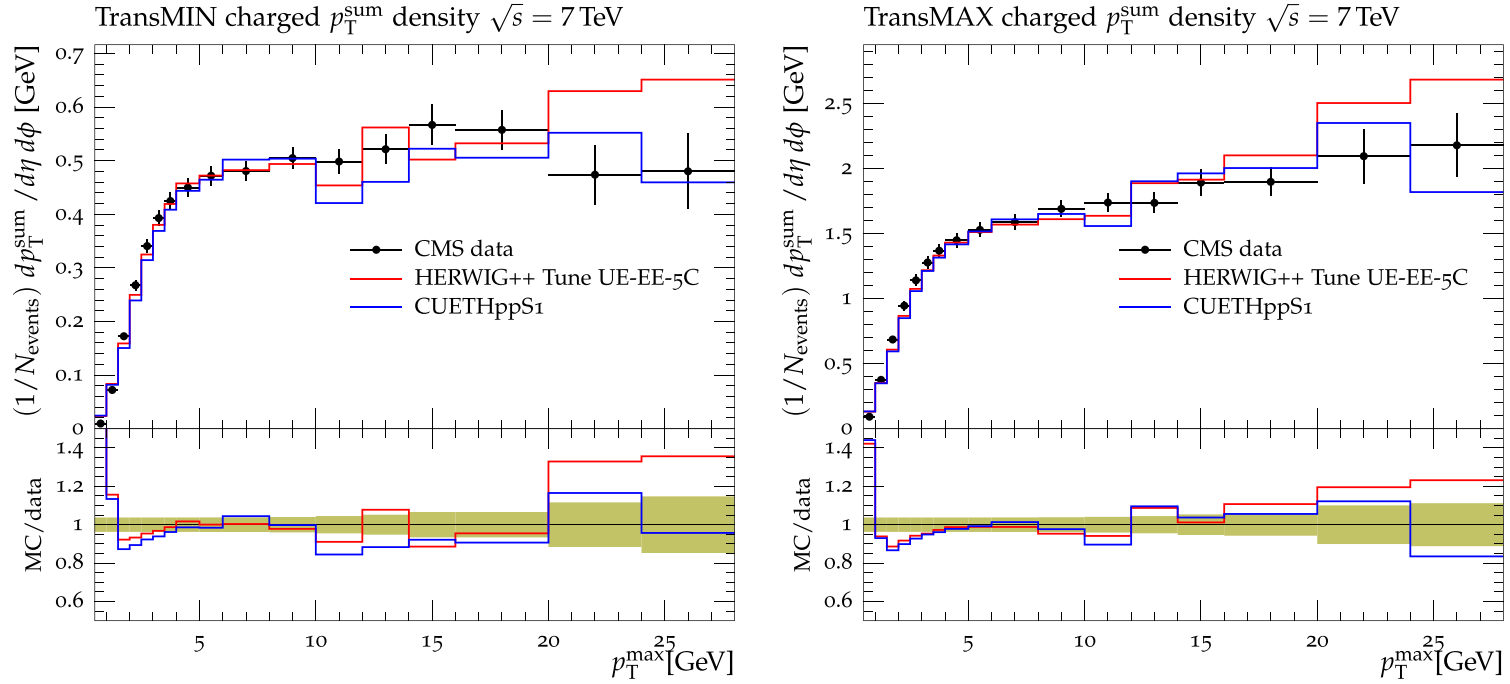

Fig. $30 \mathrm{CMS}$ data at $\sqrt{s}=7 \mathrm{TeV}$ [17] on particle (top) and $p_{\mathrm{T}}^{\text {sum }} \mathrm{den}$ sities (bottom) for charged particles with $p_{\mathrm{T}}>0.5 \mathrm{GeV}$ and $|\eta|<0.8$ in the TransMIN (left) and TransMAX (right) regions as defined by the leading charged particle, as a function of the transverse momen-

tum of the leading charged-particle $p_{\mathrm{T}}^{\max }$. The data are compared to the HERWIG++ Tune UE-EE-5C and CUETHppS1. The green bands in the ratios represent the total experimental uncertainties 


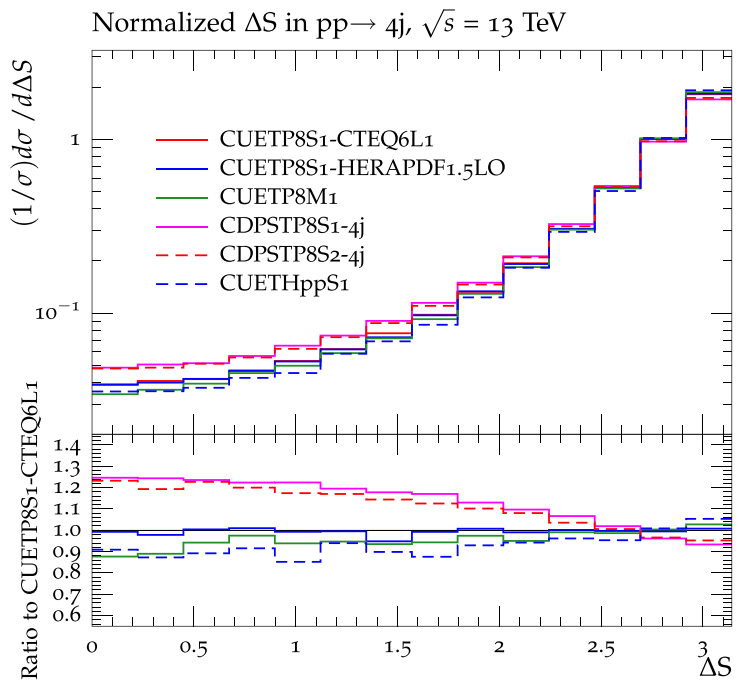

Fig. 31 Predictions at $\sqrt{s}=13 \mathrm{TeV}$ for the normalized distributions of the correlation observables $\Delta \mathrm{S}$ (left), and $\Delta^{\text {rel }} p_{\mathrm{T}}$ (right) for four-jet production in pp collisions for the three CMS PYTHIA8 UE tunes CUETP8S1-CTEQ6L1, CUETP8S1-HERAPDF1.5LO,

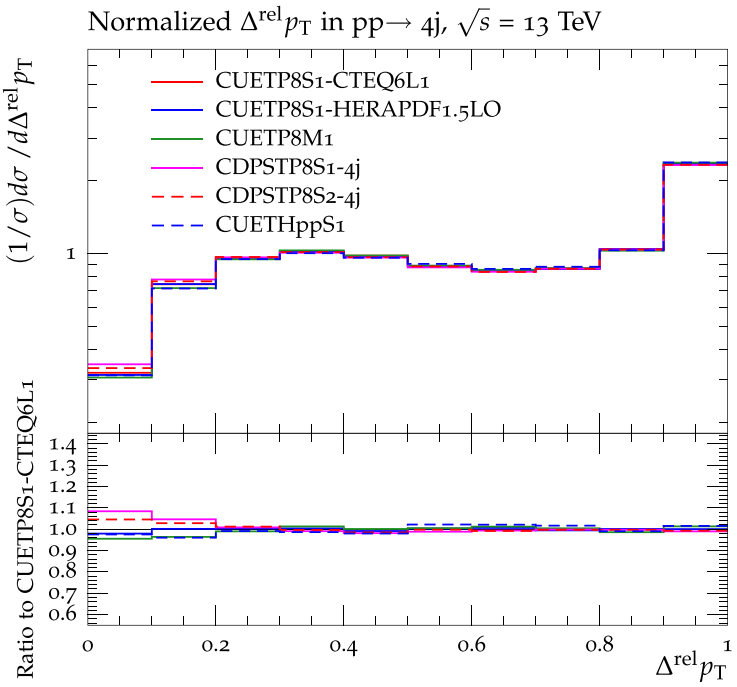

and CUETP8M1, for CUETHppS1, and for CDPSTP8S1-4j and CDPSTP8S2-4j. Also shown are the ratios of the tunes to predictions of CUETP8S1-CTEQ6L1 

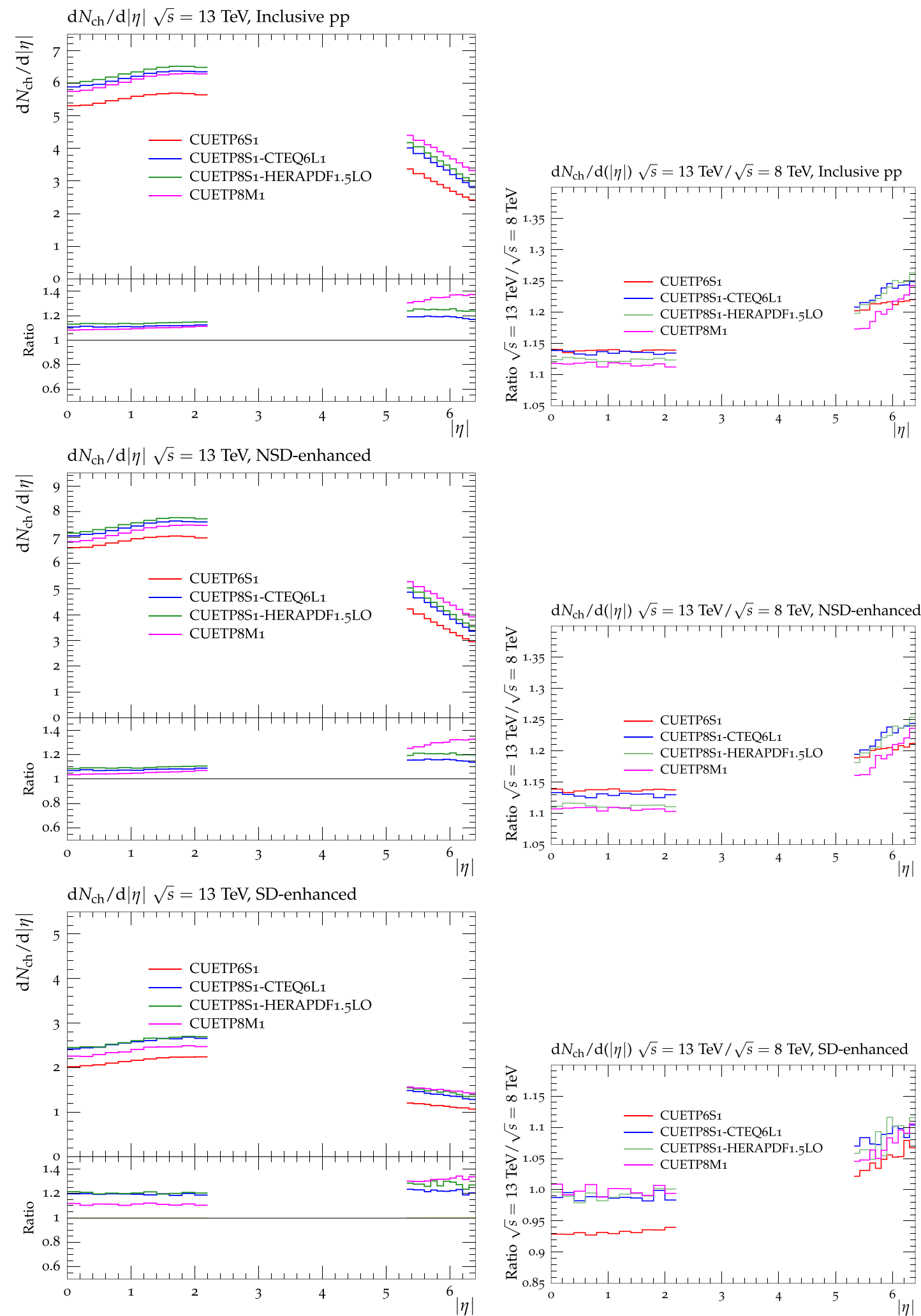

Fig. 32 Predictions at $\sqrt{s}=13 \mathrm{TeV}$ for the charged-particle pseudorapidity distribution $\mathrm{dN}_{\mathrm{ch}} / \mathrm{d} \eta$, for (top) inelastic, (middle) NSDenhanced, and (bottom) SD-enhanced pp collisions from CUETP6S1CTEQ6L1, CUETP8S1-CTEQ6L1, CUETP8S1-HERAPDF1.5LO,

and CUETP8M1. Also shown are the ratios of the tunes to predictions of CUETP8M1, and the ratio of 13 to $8 \mathrm{TeV}$ results for each of the tunes (right) 


\section{References}

1. T. Sjöstrand, S. Mrenna, P. Skands, PYTHIA 6.4 physics and manual. JHEP 05, 026 (2006). doi:10.1088/1126-6708/2006/05/026. arXiv:hep-ph/0603175

2. T. Sjöstrand, L. Lonnblad, S. Mrenna, PYTHIA 6.2: physics and manual (2001). arXiv:hep-ph/0108264

3. T. Sjöstrand, M. van Zijl, A multiple interaction model for the event structure in hadron collisions. Phys. Rev. D 36, 2019 (1987). doi:10.1103/PhysRevD.36.2019

4. M. Bengtsson, T. Sjöstrand, M. van Zijl, Initial state radiation effects on $W$ and jet production. Z. Phys. C 32, 67 (1986). doi:10. 1007/BF01441353

5. T. Sjöstrand, S. Mrenna, P.Z. Skands, A brief introduction to PYTHIA 8.1. Comput. Phys. Commun. 178, 852 (2008). doi:10. 1016/j.cpc.2008.01.036. arXiv:0710.3820

6. M. Bahr et al., Herwig++ physics and manual. Eur. Phys. J. C 58, 639 (2008). doi:10.1140/epjc/s10052-008-0798-9. arXiv:0803.0883

7. J. Bellm et al., Herwig++ 2.7 release note (2013). arXiv:1310.6877

8. M.G. Albrow et al., Tevatron-for-LHC report of the QCD working group (2006). arXiv:hep-ph/0610012

9. CDF Collaboration, Studying the 'underlying event' at CDF and the LHC. FERMILAB-CONF-09-792-E (2009)

10. P.Z. Skands, The Perugia tunes. in Proceedings, 1st International Workshop on Multiple Partonic Interactions at the LHC (MPIO8) (2009). arXiv:0905.3418

11. CDF Collaboration, A study of the energy dependence of the underlying event in proton-antiproton collisions. Phys. Rev. D 92(9), 092009 (2015). doi:10.1103/PhysRevD.92.092009. arXiv: 1508.05340

12. G.A. Schuler, T. Sjöstrand, Hadronic diffractive cross-sections and the rise of the total cross-section. Phys. Rev. D 49, 2257 (1994). doi:10.1103/PhysRevD.49.2257

13. A.V. Manohar, W.J. Waalewijn, What is double parton scattering? Phys. Lett. B 713, 196 (2012). doi:10.1016/j.physletb.2012.05.044. arXiv: 1202.5034

14. A. Del Fabbro, D. Treleani, in Multiparticle Production: New Frontiers in Soft Physics and Correlations on the Threshold of the Third Millennium. Proceedings, 9th International Workshop, Torino, Italy, June 12-17, 2000, vol. 92. Double parton scatterings at the CERN LHC (2001), p. 130. doi:10.1016/S0920-5632(00)01027-6

15. B. Blok, Y. Dokshitzer, L. Frankfurt, M. Strikman, Perturbative QCD correlations in multi-parton collisions. Eur. Phys. J. C 74, 2926 (2014). doi:10.1140/epjc/s10052-014-2926-z. arXiv: 1306.3763

16. P. Bartalini et al., Multi-parton interactions at the LHC (2011). arXiv: 1111.0469

17. CMS Collaboration, Measurement of the underlying event activity at the LHC at $7 \mathrm{TeV}$ and comparison with $0.9 \mathrm{TeV}$. CMS Physics Analysis Summary CMS-PAS-FSQ-12-020 (2012)

18. A. Buckley et al., Rivet user manual. Comput. Phys. Commun. 184, 2803 (2013). doi:10.1016/j.cpc.2013.05.021. arXiv:1003.0694

19. A. Buckley et al., Systematic event generator tuning for the LHC. Eur. Phys. J. C 65, 331 (2010). doi:10.1140/epjc/ s10052-009-1196-7. arXiv:0907.2973

20. J. Pumplin et al., New generation of parton distributions with uncertainties from global QCD analysis. JHEP 07, 012 (2002). doi:10. 1088/1126-6708/2002/07/012. arXiv:hep-ph/0201195

21. A.M. Cooper-Sarkar, in Proceedings, 22nd International Workshop on Deep-Inelastic Scattering and Related Subjects (DIS 2014), vol. DIS2014. HERAPDF1.5LO PDF set with experimental uncertainties (2014), p. 032
22. NNPDF Collaboration, Parton distributions with QED corrections. Nucl. Phys. B 877, 290 (2013). doi:10.1016/j.nuclphysb.2013.10. 010. arXiv: 1308.0598

23. NNPDF Collaboration, Unbiased global determination of parton distributions and their uncertainties at NNLO and at LO. Nucl. Phys. B 855, 153 (2012). doi:10.1016/j.nuclphysb.2011.09.024. arXiv: 1107.2652

24. CMS Collaboration, Measurement of the underlying event activity at the LHC with $\sqrt{s}=7 \mathrm{TeV}$ and comparison with $\sqrt{s}=$ 0.9 TeV. JHEP 09, 109 (2011). doi:10.1007/JHEP09(2011)109. arXiv: 1107.0330

25. CMS Collaboration, Study of the underlying event at forward rapidity in pp collisions at $\sqrt{s}=0.9,2.76$, and 7 TeV. JHEP 04, 072 (2013). doi:10.1007/JHEP04(2013)072. arXiv:1302.2394

26. CMS Collaboration, Measurement of the underlying event in the Drell-Yan process in proton-proton collisions at $\sqrt{s}=7 \mathrm{TeV}$. Eur. Phys. J. C 72, 2080 (2012). doi:10.1140/epjc/s10052-012-2080-4. arXiv: 1204.1411

27. J. Pumplin, Hard underlying event correction to inclusive jet crosssections. Phys. Rev. D 57, 5787 (1998). doi:10.1103/PhysRevD. 57.5787. arXiv:hep-ph/9708464

28. R. Corke, T. Sjöstrand, Interleaved parton showers and tuning prospects. JHEP 03, 032 (2011). doi:10.1007/JHEP03(2011)032. arXiv: 1011.1759

29. P. Skands, S. Carrazza, J. Rojo, Tuning PYTHIA 8.1: the Monash 2013 tune. Eur. Phys. J. C 74, 3024 (2014). doi:10.1140/epjc/ s10052-014-3024-y. arXiv:1404.5630

30. M.H. Seymour, A. Siodmok, Constraining MPI models using $\sigma_{\mathrm{eff}}$ and recent Tevatron and LHC underlying event data. JHEP 10, 113 (2013). doi:10.1007/JHEP10(2013)113. arXiv:1307.5015

31. P.Z. Skands, Tuning Monte Carlo generators: the Perugia tunes. Phys. Rev. D 82, 074018 (2010). doi:10.1103/PhysRevD.82. 074018. arXiv: 1005.3457

32. AFS Collaboration, Double parton scattering in pp collisions at $\sqrt{s}=63$ GeV. Z. Phys. C 34 (1987). doi:10.1007/BF01566757

33. CDF Collaboration, Double parton scattering in $\bar{p} p$ collisions at $\sqrt{s}=1.8$ TeV. Z. Phys. D 56 (1997). doi:10.1103/PhysRevD.56. 3811

34. UA2 Collaboration, A study of multi-jet events at the CERN $\bar{p} p$ collider and a search for double parton scattering. Phys. Lett. B 268, 145 (1991). doi:10.1016/0370-2693(91)90937-L

35. ATLAS Collaboration, Measurement of hard double-parton interactions in $W(\rightarrow \ell v)+2$-jet events at $\sqrt{s}=7 \mathrm{TeV}$ with the ATLAS detector. New J. Phys. 15, 033038 (2013). doi:10.1088/1367-2630/ 15/3/033038. arXiv:1301.6872

36. CMS Collaboration, Study of double parton scattering using $\mathrm{W}+$ 2-jet events in proton-proton collisions at $\sqrt{s}=7 \mathrm{TeV}$. JHEP 03, 032 (2014). doi:10.1007/JHEP03(2014)032. arXiv:1312.5729

37. CMS Collaboration, Measurement of four-jet production in proton-proton collisions at $\sqrt{s}=7 \mathrm{TeV}$. Phys. Rev. D 89, 092010 (2014). doi:10.1103/PhysRevD.89.092010. arXiv:1312.6440

38. J. Alwall et al., MadGraph 5: going beyond. JHEP 06, 128 (2011). doi:10.1007/JHEP06(2011)128. arXiv:1106.0522

39. ATLAS Collaboration, Measurement of underlying event characteristics using charged particles in $p p$ collisions at $\sqrt{s}=900 \mathrm{GeV}$ and $7 \mathrm{TeV}$ with the ATLAS detector. Phys. Rev. D 83, 112001 (2011). doi:10.1103/PhysRevD.83.112001. arXiv:1012.0791

40. B. Blok, P. Gunnellini, Dynamical approach to MPI four-jet production in Pythia. Eur. Phys. J. C 75, 282 (2015). doi:10.1140/epjc/ s10052-015-3520-8. arXiv:1503.08246

41. M. Diehl, T. Kasemets, S. Keane, Correlations in double parton distributions: effects of evolution. JHEP 05, 118 (2014). doi:10. 1007/JHEP05(2014)118. arXiv:1401.1233 
42. CMS Collaboration, Measurement of the underlying event activity using charged-particle jets in proton-proton collisions at $\sqrt{(s)}=$ 2.76 TeV. JHEP 09, 137 (2015). doi:10.1007/JHEP09(2015)137. arXiv: 1507.07229

43. B. Cooper et al., Importance of a consistent choice of $\alpha_{s}$ in the matching of AlpGen and Pythia. Eur. Phys. J. C 72, 2078 (2012). doi:10.1140/epjc/s10052-012-2078-y. arXiv:1109.5295

44. P.A. Nason, in Proceedings, 9th International Symposium on Radiative Corrections: Applications of Quantum Field Theory to Phenomenology. (RADCOR 2009), vol. RADCOR2009. Recent developments in POWHEG (2010), p. 018. arXiv:1001.2747

45. S. Alioli, P. Nason, C. Oleari, E. Re, A general framework for implementing NLO calculations in shower Monte Carlo programs: the POWHEG BOX. JHEP 06, 043 (2010). doi:10.1007/ JHEP06(2010)043. arXiv:1002.2581

46. H.-L. Lai et al., New parton distributions for collider physics. Phys. Rev. D 82, 074024 (2010). doi:10.1103/PhysRevD.82.074024. arXiv: 1007.2241

47. ALICE Collaboration, Charged-particle multiplicity measurement in proton-proton collisions at $\sqrt{s}=7 \mathrm{TeV}$ with ALICE at LHC. Eur. Phys. J. C 68, 345 (2010). doi:10.1140/epjc/ s10052-010-1350-2. arXiv:1004.3514
48. TOTEM Collaboration, First measurement of the total protonproton cross section at the LHC energy of $\sqrt{s}=7 \mathrm{TeV}$. Europhys. Lett. 96, 21002 (2011). doi:10.1209/0295-5075/96/21002. arXiv: 1110.1395

49. CMS Collaboration, Measurement of energy flow at large pseudorapidities in pp collisions at $\sqrt{s}=0.9$ and 7 TeV. JHEP 11, 148 (2011). doi:10.1007/JHEP02(2012)055. arXiv:1110.0211

50. CMS and TOTEM Collaborations, Measurement of pseudorapidity distributions of charged particles in proton-proton collisions at $\sqrt{s}=8 \mathrm{TeV}$ by the CMS and TOTEM experiments. Eur. Phys. J. C 74, 3053 (2014). doi:10.1140/epjc/s10052-014-3053-6. arXiv: 1405.0722

51. CMS Collaboration, Measurement of the inclusive jet cross section in pp collisions at $\sqrt{s}=7$ TeV. Phys. Rev. Lett. 107, 132001 (2011). doi:10.1103/PhysRevLett.107.132001. arXiv:1106.0208

52. CMS Collaboration, Measurement of the rapidity and transverse momentum distributions of $\mathrm{Z}$ bosons in pp collisions at $\sqrt{s}=7$ TeV. Phys. Rev. D 85, 032002 (2012). doi:10.1103/PhysRevD.85. 032002. arXiv: 1110.4973

53. CMS Collaboration, Pseudorapidity distribution of charged hadrons in proton-proton collisions at $\sqrt{s}=13 \mathrm{TeV}$. Phys. Lett. B 751, 143 (2015). doi:10.1016/j.physletb.2015.10.004. arXiv: 1507.05915

\section{CMS Collaboration}

\section{Yerevan Physics Institute, Yerevan, Armenia}

V. Khachatryan, A. M. Sirunyan, A. Tumasyan

\section{Institut für Hochenergiephysik der OeAW, Vienna, Austria}

W. Adam, E. Asilar, T. Bergauer, J. Brandstetter, E. Brondolin, M. Dragicevic, J. Erö, M. Flechl, M. Friedl, R. Frühwirth ${ }^{1}$, V. M. Ghete, C. Hartl, N. Hörmann, J. Hrubec, M. Jeitler ${ }^{1}$, V. Knünz, A. König, M. Krammer ${ }^{1}$, I. Krätschmer, D. Liko, T. Matsushita, I. Mikulec, D. Rabady ${ }^{2}$, B. Rahbaran, H. Rohringer, J. Schieck ${ }^{1}$, R. Schöfbeck, J. Strauss, W. Treberer-Treberspurg, W. Waltenberger, C.-E. Wulz ${ }^{1}$

\section{National Centre for Particle and High Energy Physics, Minsk, Belarus}

V. Mossolov, N. Shumeiko, J. Suarez Gonzalez

\section{Universiteit Antwerpen, Antwerp, Belgium}

S. Alderweireldt, T. Cornelis, E. A. De Wolf, X. Janssen, A. Knutsson, J. Lauwers, S. Luyckx, M. Van De Klundert, H. Van Haevermaet, P. Van Mechelen, N. Van Remortel, A. Van Spilbeeck

\section{Vrije Universiteit Brussel, Brussels, Belgium}

S. Abu Zeid, F. Blekman, J. D’Hondt, N. Daci, I. De Bruyn, K. Deroover, N. Heracleous, J. Keaveney, S. Lowette,

L. Moreels, A. Olbrechts, Q. Python, D. Strom, S. Tavernier, W. Van Doninck, P. Van Mulders, G. P. Van Onsem,

I. Van Parijs

\section{Université Libre de Bruxelles, Brussels, Belgium}

P. Barria, H. Brun, C. Caillol, B. Clerbaux, G. De Lentdecker, G. Fasanella, L. Favart, A. Grebenyuk, G. Karapostoli, T. Lenzi, A. Léonard, T. Maerschalk, A. Marinov, L. Perniè, A. Randle-conde, T. Seva, C. Vander Velde, P. Vanlaer, R. Yonamine, F. Zenoni, F. Zhang ${ }^{3}$

\section{Ghent University, Ghent, Belgium}

K. Beernaert, L. Benucci, A. Cimmino, S. Crucy, D. Dobur, A. Fagot, G. Garcia, M. Gul, J. Mccartin, A. A. Ocampo Rios, D. Poyraz, D. Ryckbosch, S. Salva, M. Sigamani, M. Tytgat, W. Van Driessche, E. Yazgan, N. Zaganidis

\section{Université Catholique de Louvain, Louvain-la-Neuve, Belgium}

S. Basegmez, C. Beluffi ${ }^{4}$, O. Bondu, S. Brochet, G. Bruno, A. Caudron, L. Ceard, G. G. Da Silveira, C. Delaere, D. Favart, L. Forthomme, A. Giammanco ${ }^{5}$, J. Hollar, A. Jafari, P. Jez, M. Komm, V. Lemaitre, A. Mertens, M. Musich, C. Nuttens, L. Perrini, A. Pin, K. Piotrzkowski, A. Popov ${ }^{6}$, L. Quertenmont, M. Selvaggi, M. Vidal Marono 
Université de Mons, Mons, Belgium

N. Beliy, G. H. Hammad

Centro Brasileiro de Pesquisas Fisicas, Rio de Janeiro, Brazil

W. L. Aldá Júnior, F. L. Alves, G. A. Alves, L. Brito, M. Correa Martins Junior, M. Hamer, C. Hensel, A. Moraes,

M. E. Pol, P. Rebello Teles

Universidade do Estado do Rio de Janeiro, Rio de Janeiro, Brazil

E. Belchior Batista Das Chagas, W. Carvalho, J. Chinellato ${ }^{7}$, A. Custódio, E. M. Da Costa, D. De Jesus Damiao,

C. De Oliveira Martins, S. Fonseca De Souza, L.M. Huertas Guativa, H. Malbouisson, D. Matos Figueiredo,

C. Mora Herrera, L. Mundim, H. Nogima, W. L. Prado Da Silva, A. Santoro, A. Sznajder, E. J. Tonelli Manganote ${ }^{7}$,

A. Vilela Pereira

Universidade Estadual Paulista ${ }^{a}$, Universidade Federal do $\mathrm{ABC}^{b}$, São Paulo, Brazil

S. Ahuja ${ }^{a}$, C. A. Bernardes ${ }^{b}$, A. De Souza Santos ${ }^{b}$, S. Dogra ${ }^{a}$, T. R. Fernandez Perez Tomei ${ }^{a}$, E. M. Gregores $^{b}$,

P. G. Mercadante ${ }^{b}$, C.S. Moon ${ }^{a}, 8$, S. F. Novaes ${ }^{a}$, Sandra S. Padula ${ }^{a}$, D. Romero Abad, J.C. Ruiz Vargas

Institute for Nuclear Research and Nuclear Energy, Sofia, Bulgaria

A. Aleksandrov, R. Hadjiiska, P. Iaydjiev, M. Rodozov, S. Stoykova, G. Sultanov, M. Vutova

University of Sofia, Sofia, Bulgaria

A. Dimitrov, I. Glushkov, L. Litov, B. Pavlov, P. Petkov

Institute of High Energy Physics, Beijing, China

M. Ahmad, J. G. Bian, G. M. Chen, H. S. Chen, M. Chen, T. Cheng, R. Du, C. H. Jiang, R. Plestina ${ }^{9}$, F. Romeo,

S. M. Shaheen,A. Spiezia, J. Tao, C. Wang, Z. Wang, H. Zhang

State Key Laboratory of Nuclear Physics and Technology, Peking University, Beijing, China

C. Asawatangtrakuldee, Y. Ban, Q. Li, S. Liu, Y. Mao, S. J. Qian, D. Wang, Z. Xu

Universidad de Los Andes, Bogota, Colombia

C. Avila, A. Cabrera, L. F. Chaparro Sierra, C. Florez, J. P. Gomez, B. Gomez Moreno, J. C. Sanabria

Faculty of Electrical Engineering, Mechanical Engineering and Naval Architecture, University of Split, Split, Croatia N. Godinovic, D. Lelas, I. Puljak, P. M. Ribeiro Cipriano

Faculty of Science, University of Split, Split, Croatia

Z. Antunovic, M. Kovac

Institute Rudjer Boskovic, Zagreb, Croatia

V. Brigljevic, K. Kadija, J. Luetic, S. Micanovic, L. Sudic

University of Cyprus, Nicosia, Cyprus

A. Attikis, G. Mavromanolakis, J. Mousa, C. Nicolaou, F. Ptochos, P. A. Razis, H. Rykaczewski

Charles University, Prague, Czech Republic

M. Bodlak, M. Finger ${ }^{10}$, M. Finger Jr. ${ }^{10}$

Academy of Scientific Research and Technology of the Arab Republic of Egypt, Egyptian Network of High Energy Physics, Cairo, Egypt

A. A. Abdelalim ${ }^{11,12}$, A. Awad, A. Mahrous ${ }^{11}$, Y. Mohammed ${ }^{13}$, A. Radi ${ }^{14,15}$

National Institute of Chemical Physics and Biophysics, Tallinn, Estonia

B. Calpas, M. Kadastik, M. Murumaa, M. Raidal, A. Tiko, C. Veelken

Department of Physics, University of Helsinki, Helsinki, Finland

P. Eerola, J. Pekkanen, M. Voutilainen

Helsinki Institute of Physics, Helsinki, Finland

J. Härkönen, V. Karimäki, R. Kinnunen, T. Lampén, K. Lassila-Perini, S. Lehti, T. Lindén, P. Luukka, T. Mäenpää,

T. Peltola, E. Tuominen, J. Tuominiemi, E. Tuovinen, L. Wendland 
Lappeenranta University of Technology, Lappeenranta, Finland

J. Talvitie, T. Tuuva

DSM/IRFU, CEA/Saclay, Gif-sur-Yvette, France

M. Besancon, F. Couderc, M. Dejardin, D. Denegri, B. Fabbro, J. L. Faure, C. Favaro, F. Ferri, S. Ganjour, A. Givernaud, P. Gras, G. Hamel de Monchenault, P. Jarry, E. Locci, M. Machet, J. Malcles, J. Rander, A. Rosowsky, M. Titov,

A. Zghiche

Laboratoire Leprince-Ringuet, Ecole Polytechnique, IN2P3-CNRS, Palaiseau, France

I. Antropov, S. Baffioni, F. Beaudette, P. Busson, L. Cadamuro, E. Chapon, C. Charlot, T. Dahms, O. Davignon,

N. Filipovic, R. Granier de Cassagnac, M. Jo, S. Lisniak, L. Mastrolorenzo, P. Miné, I. N. Naranjo, M. Nguyen,

C. Ochando, G. Ortona, P. Paganini, P. Pigard, S. Regnard, R. Salerno, J. B. Sauvan, Y. Sirois, T. Strebler, Y. Yilmaz,

A. Zabi

Institut Pluridisciplinaire Hubert Curien, Université de Strasbourg, Université de Haute Alsace Mulhouse, CNRS/IN2P3, Strasbourg, France

J.-L. Agram ${ }^{16}$, J. Andrea, A. Aubin, D. Bloch, J.-M. Brom, M. Buttignol, E. C. Chabert, N. Chanon, C. Collard,

E. Conte ${ }^{16}$, X. Coubez, J.-C. Fontaine ${ }^{16}$, D. Gelé, U. Goerlach, C. Goetzmann, A.-C. Le Bihan, J. A. Merlin ${ }^{2}$, K. Skovpen,

P. Van Hove

Centre de Calcul de l'Institut National de Physique Nucleaire et de Physique des Particules, CNRS/IN2P3, Villeurbanne, France

S. Gadrat

Institut de Physique Nucléaire de Lyon, Université de Lyon, Université Claude Bernard Lyon 1, CNRS-IN2P3, Villeurbanne, France

S. Beauceron, C. Bernet, G. Boudoul, E. Bouvier, C. A. Carrillo Montoya, R. Chierici, D. Contardo, B. Courbon, P. Depasse, H. El Mamouni, J. Fan, J. Fay, S. Gascon, M. Gouzevitch, B. Ille, F. Lagarde, I. B. Laktineh, M. Lethuillier, L. Mirabito, A. L. Pequegnot, S. Perries, J. D. Ruiz Alvarez, D. Sabes, L. Sgandurra, V. Sordini, M. Vander Donckt, P. Verdier, S. Viret

Georgian Technical University, Tbilisi, Georgia

T. Toriashvili ${ }^{17}$

Tbilisi State University, Tbilisi, Georgia

D. Lomidze

I. Physikalisches Institut, RWTH Aachen University, Aachen, Germany

C. Autermann, S. Beranek, M. Edelhoff, L. Feld, A. Heister, M. K. Kiesel, K. Klein, M. Lipinski, A. Ostapchuk,

M. Preuten, F. Raupach, S. Schael, J. F. Schulte, T. Verlage, H. Weber, B. Wittmer, V. Zhukov ${ }^{6}$

III. Physikalisches Institut A, RWTH Aachen University, Aachen, Germany

M. Ata, M. Brodski, E. Dietz-Laursonn, D. Duchardt, M. Endres, M. Erdmann, S. Erdweg, T. Esch, R. Fischer, A. Güth, T. Hebbeker, C. Heidemann, K. Hoepfner, S. Knutzen, P. Kreuzer, M. Merschmeyer, A. Meyer, P. Millet, M. Olschewski, K. Padeken, P. Papacz, T. Pook, M. Radziej, H. Reithler, M. Rieger, F. Scheuch, L. Sonnenschein, D. Teyssier, S. Thüer

III. Physikalisches Institut B, RWTH Aachen University, Aachen, Germany

V. Cherepanov, Y. Erdogan, G. Flügge, H. Geenen, M. Geisler, F. Hoehle, B. Kargoll, T. Kress, Y. Kuessel, A. Künsken, J. Lingemann, A. Nehrkorn, A. Nowack, I. M. Nugent, C. Pistone, O. Pooth, A. Stahl

\section{Deutsches Elektronen-Synchrotron, Hamburg, Germany}

M. Aldaya Martin, I. Asin, N. Bartosik, O. Behnke, U. Behrens, A. J. Bell, K. Borras ${ }^{18}$, A. Burgmeier, A. Campbell,

S. Choudhury ${ }^{19}$, F. Costanza, C. Diez Pardos, G. Dolinska, S. Dooling, T. Dorland, G. Eckerlin, D. Eckstein, T. Eichhorn, G. Flucke, E. Gallo ${ }^{20}$, J. Garay Garcia, A. Geiser, A. Gizhko, P. Gunnellini, J. Hauk, M. Hempel ${ }^{21}$, H. Jung,

A. Kalogeropoulos, O. Karacheban ${ }^{21}$, M. Kasemann, P. Katsas, J. Kieseler, C. Kleinwort, I. Korol, W. Lange, J. Leonard, K. Lipka, A. Lobanov, W. Lohmann ${ }^{21}$, R. Mankel, I. Marfin ${ }^{21}$, I.-A. Melzer-Pellmann, A. B. Meyer, G. Mittag, J. Mnich, A. Mussgiller, S. Naumann-Emme, A. Nayak, E. Ntomari, H. Perrey, D. Pitzl, R. Placakyte, A. Raspereza, B. Roland, 
M. Ö. Sahin, P. Saxena, T. Schoerner-Sadenius, M. Schröder, C. Seitz, S. Spannagel, K. D. Trippkewitz, R. Walsh,

C. Wissing

\section{University of Hamburg, Hamburg, Germany}

V. Blobel, M. Centis Vignali, A. R. Draeger, J. Erfle, E. Garutti, K. Goebel, D. Gonzalez, M. Görner, J. Haller, M. Hoffmann, R. S. Höing, A. Junkes, R. Klanner, R. Kogler, N. Kovalchuk, T. Lapsien, T. Lenz, I. Marchesini, D. Marconi, M. Meyer, D. Nowatschin, J. Ott, F. Pantaleo ${ }^{2}$, T. Peiffer, A. Perieanu, N. Pietsch, J. Poehlsen, D. Rathjens, C. Sander,C. Scharf, H. Schettler, P. Schleper, E. Schlieckau, A. Schmidt, J. Schwandt, V. Sola, H. Stadie, G. Steinbrück, H. Tholen, D. Troendle, E. Usai, L. Vanelderen, A. Vanhoefer, B. Vormwald

\section{Institut für Experimentelle Kernphysik, Karlsruhe, Germany}

C. Barth, C. Baus, J. Berger, C. Böser, E. Butz, T. Chwalek, F. Colombo, W. De Boer, A. Descroix, A. Dierlamm, S. Fink, F. Frensch, R. Friese, M. Giffels, A. Gilbert, D. Haitz, F. Hartmann², S. M. Heindl, U. Husemann, I. Katkov' ${ }^{6}$, A. Kornmayer ${ }^{2}$, P. Lobelle Pardo, B. Maier, H. Mildner, M. U. Mozer, T. Müller, Th. Müller, M. Plagge, G. Quast, K. Rabbertz, S. Röcker, F. Roscher, G. Sieber, H. J. Simonis, F. M. Stober, R. Ulrich, J. Wagner-Kuhr, S. Wayand, M. Weber, T. Weiler, S. Williamson, C. Wöhrmann, R. Wolf

Institute of Nuclear and Particle Physics (INPP), NCSR Demokritos, Aghia Paraskevi, Greece G. Anagnostou, G. Daskalakis, T. Geralis, V. A. Giakoumopoulou, A. Kyriakis, D. Loukas, A. Psallidas, I. Topsis-Giotis

University of Athens, Athens, Greece

A. Agapitos, S. Kesisoglou, A. Panagiotou, N. Saoulidou, E. Tziaferi

University of Ioánnina, Ioánnina, Greece

I. Evangelou, G. Flouris, C. Foudas, P. Kokkas, N. Loukas, N. Manthos, I. Papadopoulos, E. Paradas, J. Strologas

Wigner Research Centre for Physics, Budapest, Hungary

G. Bencze, C. Hajdu, A. Hazi, P. Hidas, D. Horvath ${ }^{22}$, F. Sikler, V. Veszpremi, G. Vesztergombi ${ }^{23}$, A. J. Zsigmond

Institute of Nuclear Research ATOMKI, Debrecen, Hungary

N. Beni, S. Czellar, J. Karancsi ${ }^{24}$, J. Molnar, Z. Szillasi ${ }^{2}$

University of Debrecen, Debrecen, Hungary

M. Bartók ${ }^{25}$, A. Makovec, P. Raics, Z. L. Trocsanyi, B. Ujvari

National Institute of Science Education and Research, Bhubaneswar, India

P. Mal, K. Mandal, D. K. Sahoo, N. Sahoo, S. K. Swain

Panjab University, Chandigarh, India

S. Bansal, S. B. Beri, V. Bhatnagar, R. Chawla, R. Gupta, U. Bhawandeep, A. K. Kalsi, A. Kaur, M. Kaur, R. Kumar, A. Mehta, M. Mittal, J. B. Singh, G. Walia

University of Delhi, Delhi, India

Ashok Kumar, A. Bhardwaj, B. C. Choudhary, R. B. Garg, A. Kumar, S. Malhotra, M. Naimuddin, N. Nishu, K. Ranjan, R. Sharma, V. Sharma

Saha Institute of Nuclear Physics, Kolkata, India

S. Bhattacharya, K. Chatterjee, S. Dey, S. Dutta, Sa. Jain, N. Majumdar, A. Modak, K. Mondal, S. Mukherjee,

S. Mukhopadhyay, A. Roy, D. Roy, S. Roy Chowdhury, S. Sarkar, M. Sharan

Bhabha Atomic Research Centre, Mumbai, India

A. Abdulsalam, R. Chudasama, D. Dutta, V. Jha, V. Kumar, A. K. Mohanty ${ }^{2}$, L. M. Pant, P. Shukla, A. Topkar

Tata Institute of Fundamental Research, Mumbai, India

T. Aziz, S. Banerjee, S. Bhowmik ${ }^{26}$, R. M. Chatterjee, R. K. Dewanjee, S. Dugad, S. Ganguly, S. Ghosh, M. Guchait,

A. Gurtu ${ }^{27}$, G. Kole, S. Kumar, B. Mahakud, M. Maity ${ }^{26}$, G. Majumder, K. Mazumdar, S. Mitra, G. B. Mohanty, B. Parida, T. Sarkar ${ }^{26}$, N. Sur,B. Sutar, N. Wickramage 28

Indian Institute of Science Education and Research (IISER), Pune, India

S. Chauhan, S. Dube, A. Kapoor, K. Kothekar, S. Sharma 
Institute for Research in Fundamental Sciences (IPM), Tehran, Iran

H. Bakhshiansohi, H. Behnamian, S. M. Etesami ${ }^{29}$, A. Fahim ${ }^{30}$, R. Goldouzian, M. Khakzad, M. Mohammadi Najafabadi, M. Naseri, S. Paktinat Mehdiabadi, F. Rezaei Hosseinabadi, B. Safarzadeh ${ }^{31}$, M. Zeinali

\section{University College Dublin, Dublin, Ireland}

M. Felcini, M. Grunewald

INFN Sezione di Bari ${ }^{a}$, Università di Bari ${ }^{b}$, Politecnico di Bari $^{c}$, Bari, Italy

M. Abbrescia ${ }^{a, b}$, C. Calabria ${ }^{a, b}$, C. Caputo ${ }^{a, b}$, A. Colaleo $^{a}$, D. Creanza ${ }^{a, c}$, L. Cristella $^{a, b}$, N. De Filippis ${ }^{a, c}$,

M. De Palma ${ }^{a, b}$, L. Fiore ${ }^{a}$, G. Iaselli ${ }^{a, c}$, G. Maggi ${ }^{a, c}$, M. Maggi $^{a}$, G. Miniello ${ }^{a, b}$, S. My $^{a, c}$, S. Nuzzo $^{a, b}$, A. Pompili ${ }^{a, b}$,

G. Pugliese $^{a, c}$, R. Radogna ${ }^{a, b}$, A. Ranieri ${ }^{a}$, G. Selvaggi ${ }^{a, b}$, L. Silvestris $^{a, 2}$, R. Venditti ${ }^{a, b}$, P. Verwilligen $^{a}$

INFN Sezione di Bologna ${ }^{a}$, Università di Bologna ${ }^{b}$, Bologna, Italy

G. Abbiendi ${ }^{a}$, C. Battilana ${ }^{2}$, A. C. Benvenuti ${ }^{a}$, D. Bonacorsi ${ }^{a}, b$, S. Braibant-Giacomellia ${ }^{a, b}$, L. Brigliadori ${ }^{a}, b$,

R. Campanini ${ }^{a, b}$, P. Capiluppi ${ }^{a}, b$, A. Castro ${ }^{a, b}$, F. R. Cavallo ${ }^{a}$, S. S. Chhibra ${ }^{a, b}$, G. Codispoti $^{a, b}$, M. Cuffiani $^{a, b}$,

G. M. Dallavalle ${ }^{a}$, F. Fabbri ${ }^{a}$, A. Fanfani ${ }^{a, b}$, D. Fasanella ${ }^{a, b}$, P. Giacomelli ${ }^{a}$, C. Grandi ${ }^{a}$, L. Guiducci ${ }^{a}, b$, S. Marcellini ${ }^{a}$,

G. Masetti ${ }^{a}$, A. Montanari ${ }^{a}$, F. L. Navarria ${ }^{a, b}$, A. Perrotta ${ }^{a}$, A. M. Rossi ${ }^{a, b}$, T. Rovelli ${ }^{a, b}$, G. P. Siroli ${ }^{a}, b$, N. Tosi ${ }^{a, b, 2}$,

R. Travaglini ${ }^{a, b}$

INFN Sezione di Catania ${ }^{a}$, Università di Catania ${ }^{b}$, Catania, Italy

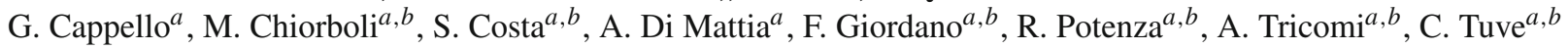

INFN Sezione di Firenze ${ }^{a}$, Università di Firenze ${ }^{b}$, Florence, Italy

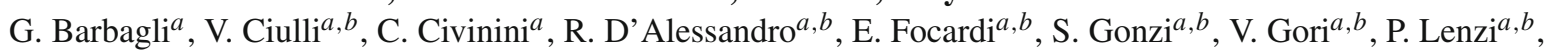

M. Meschini ${ }^{a}$, S. Paoletti $^{a}$, G. Sguazzoni $^{a}$, A. Tropiano $^{a, b}$, L. Viliani $^{a, b, 2}$

INFN Laboratori Nazionali di Frascati, Frascati, Italy

L. Benussi, S. Bianco, F. Fabbri, D. Piccolo, F. Primavera ${ }^{2}$

INFN Sezione di Genova ${ }^{a}$, Università di Genova ${ }^{b}$, Genoa, Italy

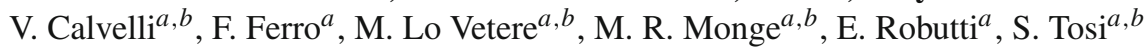

INFN Sezione di Milano-Bicocca ${ }^{a}$, Università di Milano-Bicocca ${ }^{b}$, Milan, Italy

L. Brianza, M. E. Dinardo ${ }^{a, b}$, S. Fiorendi ${ }^{a, b}$, S. Gennai ${ }^{a}$, R. Gerosa ${ }^{a, b}$, A. Ghezzi ${ }^{a}, b$, P. Govoni $^{a, b}$, S. Malvezzi ${ }^{a}$,

R. A. Manzoni ${ }^{a, b, 2}$, B. Marzocchi ${ }^{a, b, 2}$, D. Menasce ${ }^{a}$, L. Moroni $^{a}$, M. Paganoni ${ }^{a, b}$, D. Pedrini ${ }^{a}$, S. Ragazzi $^{a, b}$,

N. Redaelli ${ }^{a}$, T. Tabarelli de Fatis ${ }^{a, b}$

INFN Sezione di Napoli ${ }^{a}$, Università di Napoli 'Federico II' ${ }^{b}$, Napoli, Italy, Università della Basilicata ${ }^{c}$, Potenza, Italy, Università G. Marconi ${ }^{d}$, Rome, Italy

S. Buontempo ${ }^{a}$, N. Cavallo ${ }^{a, c}$, S. Di Guida ${ }^{a, d, 2}$, M. Esposito ${ }^{a, b}$, F. Fabozzi ${ }^{a, c}$, A. O. M. Iorio ${ }^{a, b}$, G. Lanza ${ }^{a}$ L. Lista $^{a}$, S. Meola ${ }^{a, d, 2}$, M. Merola ${ }^{a}$, P. Paolucci ${ }^{a, 2}$, C. Sciacca ${ }^{a, b}$, F. Thyssen

INFN Sezione di Padova ${ }^{a}$, Università di Padova ${ }^{b}$, Padova, Italy, Università di Trento $^{c}$, Trento, Italy

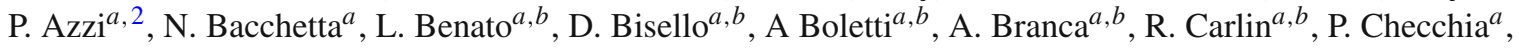

M. Dall'Osso ${ }^{a, b, 2}$, T. Dorigo ${ }^{a}$, U. Dosselli $^{a}$, S. Fantinel ${ }^{a}$, F. Fanzago $^{a}$, F. Gasparini ${ }^{a, b}$, U. Gasparini ${ }^{a, b}$, A. Gozzelino $^{a}$,

K. Kanishchev ${ }^{a, c}$, S. Lacaprara ${ }^{a}$, M. Margoni ${ }^{a, b}$, A. T. Meneguzzo ${ }^{a, b}$, J. Pazzini $^{a, b, 2}$, N. Pozzobon $^{a, b}$, P. Ronchese ${ }^{a, b}$,

F. Simonetto ${ }^{a, b}$, E. Torassa $^{a}$, M. Tosi ${ }^{a, b}$, M. Zanetti, P. Zotto ${ }^{a, b}$, A. Zucchetta $^{a, b, 2}$

INFN Sezione di Pavia ${ }^{a}$, Università di Pavia ${ }^{b}$, Pavia, Italy

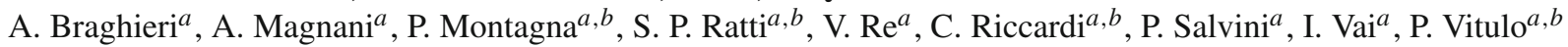

INFN Sezione di Perugia ${ }^{a}$, Università di Perugia ${ }^{b}$, Perugia, Italy

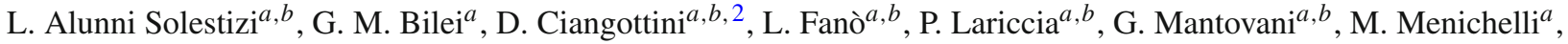

A. Saha ${ }^{a}$, A. Santocchia ${ }^{a, b}$

INFN Sezione di Pisa ${ }^{a}$, Università di Pisa ${ }^{b}$, Scuola Normale Superiore di Pisa ${ }^{c}$, Pisa, Italy

K. Androsov $a, 32$, P. Azzurri ${ }^{a, 2}$, G. Bagliesi ${ }^{a}$, J. Bernardini ${ }^{a}$, T. Boccali ${ }^{a}$, R. Castaldi $^{a}$, M. A. Ciocci ${ }^{a, 32}$, R. Dell'Orso ${ }^{a}$,

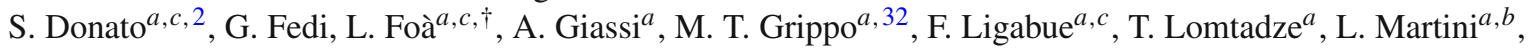




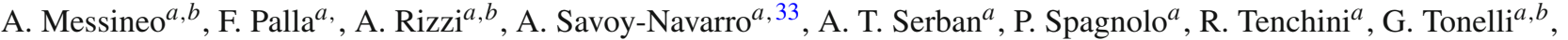

A. Venturi ${ }^{a}$, P. G. Verdini ${ }^{a}$

INFN Sezione di Roma ${ }^{a}$, Università di Roma ${ }^{b}$, Rome, Italy

L. Barone ${ }^{a, b}$, F. Cavallari ${ }^{a}$, G. D'imperio ${ }^{a, b, 2}$, D. Del Re ${ }^{a, b, 2}$, M. Diemoz ${ }^{a}$, S. Gelli ${ }^{a, b}$, C. Jorda ${ }^{a}$, E. Longo ${ }^{a, b}$,

F. Margaroli ${ }^{a, b}$, P. Meridiani ${ }^{a}$, G. Organtini ${ }^{a, b}$, R. Paramatti ${ }^{a}$, F. Preiato ${ }^{a, b}$, S. Rahatlou ${ }^{a, b}$, C. Rovelli $^{a}$, F. Santanastasio $^{a, b}$,

P. Traczyk ${ }^{a, b, 2}$

INFN Sezione di Torino ${ }^{a}$, Università di Torino ${ }^{b}$, Torino, Italy, Università del Piemonte Orientale ${ }^{c}$, Novara, Italy

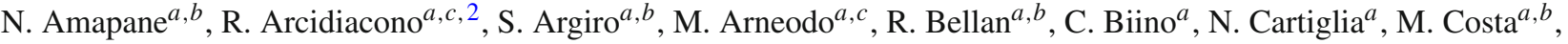

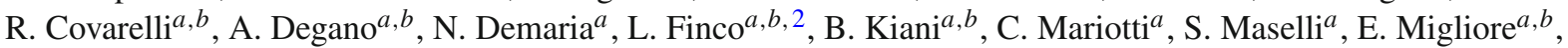

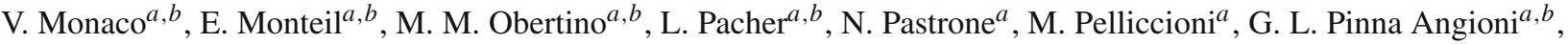

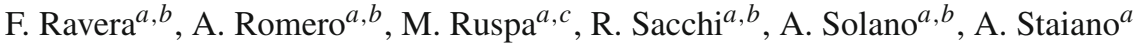

INFN Sezione di Trieste ${ }^{a}$, Università di Trieste ${ }^{b}$, Trieste, Italy

S. Belforte ${ }^{a}$, V. Candelise ${ }^{a, b, 2}$, M. Casarsa ${ }^{a}$, F. Cossutti ${ }^{a}$, G. Della Ricca ${ }^{a, b}$, B. Gobbo ${ }^{a}$, C. La Licata ${ }^{a, b}$, M. Marone ${ }^{a, b}$, A. Schizzi ${ }^{a, b}$, A. Zanetti ${ }^{a}$

\section{Kangwon National University, Chunchon, Korea}

A. Kropivnitskaya, S. K. Nam

Kyungpook National University, Daegu, Korea

D. H. Kim, G. N. Kim, M. S. Kim, D. J. Kong, S. Lee, Y. D. Oh, A. Sakharov, D. C. Son

Chonbuk National University, Jeonju, Korea

J. A. Brochero Cifuentes, H. Kim, T. J. Kim

Chonnam National University, Institute for Universe and Elementary Particles, Kwangju, Korea

S. Song

Korea University, Seoul, Korea

S. Choi, Y. Go, D. Gyun, B. Hong, H. Kim, Y. Kim, B. Lee, K. Lee, K. S. Lee, S. Lee, S. K. Park, Y. Roh

Seoul National University, Seoul, Korea

H. D. Yoo

University of Seoul, Seoul, Korea

M. Choi, H. Kim, J. H. Kim, J. S. H. Lee, I. C. Park, G. Ryu, M. S. Ryu

Sungkyunkwan University, Suwon, Korea

Y. Choi, J. Goh, D. Kim, E. Kwon, J. Lee, I. Yu

Vilnius University, Vilnius, Lithuania

V. Dudenas, A. Juodagalvis, J. Vaitkus

National Centre for Particle Physics, Universiti Malaya, Kuala Lumpur, Malaysia

I. Ahmed, Z. A. Ibrahim, J. R. Komaragiri, M. A. B. Md Ali $^{34}$, F. Mohamad Idris ${ }^{35}$, W. A. T. Wan Abdullah, M. N. Yusli

Centro de Investigacion y de Estudios Avanzados del IPN, Mexico City, Mexico

E. Casimiro Linares, H. Castilla-Valdez, E. De La Cruz-Burelo, I. Heredia-De La Cruz ${ }^{36}$, A. Hernandez-Almada,

R. Lopez-Fernandez, A. Sanchez-Hernandez

Universidad Iberoamericana, Mexico City, Mexico

S. Carrillo Moreno, F. Vazquez Valencia

Benemerita Universidad Autonoma de Puebla, Puebla, Mexico

I. Pedraza, H. A. Salazar Ibarguen

Universidad Autónoma de San Luis Potosí, San Luis Potosí, Mexico

A. Morelos Pineda 
University of Auckland, Auckland, New Zealand

D. Krofcheck

University of Canterbury, Christchurch, New Zealand

P. H. Butler

National Centre for Physics, Quaid-I-Azam University, Islamabad, Pakistan

A. Ahmad, M. Ahmad, Q. Hassan, H. R. Hoorani, W. A. Khan, T. Khurshid, M. Shoaib

National Centre for Nuclear Research, Swierk, Poland

H. Bialkowska, M. Bluj, B. Boimska, T. Frueboes, M. Górski, M. Kazana, K. Nawrocki, K. Romanowska-Rybinska,

M. Szleper, P. Zalewski

Institute of Experimental Physics, Faculty of Physics, University of Warsaw, Warsaw, Poland

G. Brona, K. Bunkowski, A. Byszuk ${ }^{37}$, K. Doroba, A. Kalinowski, M. Konecki, J. Krolikowski, M. Misiura, M. Olszewski, M. Walczak

Laboratório de Instrumentação e Física Experimental de Partículas, Lisbon, Portugal

P. Bargassa, C. Beirão Da Cruz E Silva, A. Di Francesco, P. Faccioli, P. G. Ferreira Parracho, M. Gallinaro, N. Leonardo,

L. Lloret Iglesias, F. Nguyen, J. Rodrigues Antunes, J. Seixas, O. Toldaiev, D. Vadruccio, J. Varela, P. Vischia

Joint Institute for Nuclear Research, Dubna, Russia

S. Afanasiev, P. Bunin, M. Gavrilenko, I. Golutvin, I. Gorbunov, A. Kamenev, V. Karjavin, V. Konoplyanikov, A. Lanev,

A. Malakhov, V. Matveev ${ }^{38,39}$, P. Moisenz, V. Palichik, V. Perelygin, S. Shmatov, S. Shulha, N. Skatchkov, V. Smirnov,

A. Zarubin

Petersburg Nuclear Physics Institute, Gatchina, St. Petersburg, Russia

V. Golovtsov, Y. Ivanov, V. Kim ${ }^{40}$, E. Kuznetsova, P. Levchenko, V. Murzin, V. Oreshkin, I. Smirnov, V. Sulimov,

L. Uvarov, S. Vavilov, A. Vorobyev

Institute for Nuclear Research, Moscow, Russia

Yu. Andreev, A. Dermenev, S. Gninenko, N. Golubev, A. Karneyeu, M. Kirsanov, N. Krasnikov, A. Pashenkov, D. Tlisov, A. Toropin

Institute for Theoretical and Experimental Physics, Moscow, Russia

V. Epshteyn, V. Gavrilov, N. Lychkovskaya, V. Popov, I. Pozdnyakov, G. Safronov, A. Spiridonov, E. Vlasov, A. Zhokin

National Research Nuclear University 'Moscow Engineering Physics Institute'(MEPhI), Moscow, Russia

A. Bylinkin

P. N. Lebedev Physical Institute, Moscow, Russia

V. Andreev, M. Azarkin ${ }^{39}$, I. Dremin ${ }^{39}$, M. Kirakosyan, A. Leonidov ${ }^{39}$, G. Mesyats, S. V. Rusakov

Skobeltsyn Institute of Nuclear Physics, Lomonosov Moscow State University, Moscow, Russia

A. Baskakov, A. Belyaev, E. Boos, M. Dubinin ${ }^{41}$, L. Dudko, A. Ershov, A. Gribushin, V. Klyukhin, O. Kodolova,

I. Lokhtin, I. Myagkov, S. Obraztsov, S. Petrushanko, V. Savrin, A. Snigirev

State Research Center of Russian Federation, Institute for High Energy Physics, Protvino, Russia

I. Azhgirey, I. Bayshev, S. Bitioukov, V. Kachanov, A. Kalinin, D. Konstantinov, V. Krychkine, V. Petrov, R. Ryutin,

A. Sobol, L. Tourtchanovitch, S. Troshin, N. Tyurin, A. Uzunian, A. Volkov

Faculty of Physics and Vinca Institute of Nuclear Sciences, University of Belgrade, Belgrade, Serbia P. Adzic ${ }^{42}$, P. Cirkovic, J. Milosevic, V. Rekovic

Centro de Investigaciones Energéticas Medioambientales y Tecnológicas (CIEMAT), Madrid, Spain J. Alcaraz Maestre, E. Calvo, M. Cerrada, M. Chamizo Llatas, N. Colino, B. De La Cruz, A. Delgado Peris, A. Escalante Del Valle, C. Fernandez Bedoya, J. P. Fernández Ramos, J. Flix, M. C. Fouz, P. Garcia-Abia,

O. Gonzalez Lopez, S. Goy Lopez, J. M. Hernandez, M. I. Josa, E. Navarro De Martino, A. Pérez-Calero Yzquierdo,

J. Puerta Pelayo, A. Quintario Olmeda, I. Redondo, L. Romero, J. Santaolalla, M. S. Soares 
Universidad Autónoma de Madrid, Madrid, Spain

C. Albajar, J. F. de Trocóniz, M. Missiroli, D. Moran

Universidad de Oviedo, Oviedo, Spain

J. Cuevas, J. Fernandez Menendez, S. Folgueras, I. Gonzalez Caballero, E. Palencia Cortezon, J. M. Vizan Garcia

Instituto de Física de Cantabria (IFCA), CSIC-Universidad de Cantabria, Santander, Spain

I. J. Cabrillo, A. Calderon, J. R. Castiñeiras De Saa, P. De Castro Manzano, M. Fernandez, J. Garcia-Ferrero, G. Gomez, A. Lopez Virto, J. Marco, R. Marco, C. Martinez Rivero, F. Matorras, J. Piedra Gomez, T. Rodrigo,

A. Y. Rodríguez-Marrero, A. Ruiz-Jimeno, L. Scodellaro, N. Trevisani, I. Vila, R. Vilar Cortabitarte

\section{CERN, European Organization for Nuclear Research, Geneva, Switzerland}

D. Abbaneo, E. Auffray, G. Auzinger, M. Bachtis, P. Baillon, A. H. Ball, D. Barney, A. Benaglia, J. Bendavid,

L. Benhabib, J. F. Benitez, G. M. Berruti, P. Bloch, A. Bocci, A. Bonato, C. Botta, H. Breuker, T. Camporesi, R. Castello,

G. Cerminara, M. D'Alfonso, D. d'Enterria, A. Dabrowski, V. Daponte, A. David, M. De Gruttola, F. De Guio,

A. De Roeck, S. De Visscher, E. Di Marco ${ }^{43}$, M. Dobson, M. Dordevic, B. Dorney, T. du Pree, D. Duggan, M. Dünser,

N. Dupont, A. Elliott-Peisert, G. Franzoni, J. Fulcher, W. Funk, D. Gigi, K. Gill, D. Giordano, M. Girone, F. Glege,

R. Guida, S. Gundacker, M. Guthoff, J. Hammer, P. Harris, J. Hegeman, V. Innocente, P. Janot, H. Kirschenmann,

M. J. Kortelainen, K. Kousouris, K. Krajczar, P. Lecoq, C. Lourenço,M. T. Lucchini, N. Magini, L. Malgeri, M. Mannelli,

A. Martelli, L. Masetti, F. Meijers, S. Mersi, E. Meschi, F. Moortgat, S. Morovic, M. Mulders, M. V. Nemallapudi,

H. Neugebauer, S. Orfanelli ${ }^{44}$, L. Orsini, L. Pape, E. Perez, M. Peruzzi, A. Petrilli, G. Petrucciani, A. Pfeiffer, D. Piparo,

A. Racz, T. Reis, G. Rolandi ${ }^{45}$, M. Rovere, M. Ruan, H. Sakulin, C. Schäfer, C. Schwick, M. Seidel, A. Sharma, P. Silva,

M. Simon, P. Sphicas ${ }^{46}$, J. Steggemann, B. Stieger, M. Stoye, Y. Takahashi, D. Treille, A. Triossi, A. Tsirou, G. I. Veres ${ }^{23}$,

N. Wardle, H. K. Wöhri, A. Zagozdzinska ${ }^{37}$, W. D. Zeuner

Paul Scherrer Institut, Villigen, Switzerland

W. Bertl, K. Deiters, W. Erdmann, R. Horisberger, Q. Ingram, H. C. Kaestli, D. Kotlinski, U. Langenegger, D. Renker,

T. Rohe

Institute for Particle Physics, ETH Zurich, Zurich, Switzerland

F. Bachmair, L. Bäni, L. Bianchini, B. Casal, G. Dissertori, M. Dittmar, M. Donegà, P. Eller, C. Grab, C. Heidegger,

D. Hits, J. Hoss, G. Kasieczka, W. Lustermann, B. Mangano, M. Marionneau, P. Martinez Ruiz del Arbol,

M. Masciovecchio, D. Meister, F. Micheli, P. Musella, F. Nessi-Tedaldi, F. Pandolfi, J. Pata, F. Pauss, L. Perrozzi,

M. Quittnat, M. Rossini, A. Starodumov ${ }^{47}$, M. Takahashi, V. R. Tavolaro, K. Theofilatos, R. Wallny

Universität Zürich, Zurich, Switzerland

T. K. Aarrestad, C. Amsler ${ }^{48}$, L. Caminada, M. F. Canelli, V. Chiochia, A. De Cosa, C. Galloni, A. Hinzmann, T. Hreus,

B. Kilminster, C. Lange, J. Ngadiuba, D. Pinna, P. Robmann, F. J. Ronga, D. Salerno, Y. Yang

National Central University, Chung-Li, Taiwan

M. Cardaci, K. H. Chen, T. H. Doan, Sh. Jain, R. Khurana, M. Konyushikhin, C. M. Kuo, W. Lin, Y. J. Lu, S. S. Yu

National Taiwan University (NTU), Taipei, Taiwan

Arun Kumar, R. Bartek, P. Chang, Y. H. Chang, Y. W. Chang, Y. Chao, K. F. Chen, P. H. Chen, C. Dietz, F. Fiori,

U. Grundler, W.-S. Hou, Y. Hsiung, Y. F. Liu, R.-S. Lu, M. Miñano Moya, E. Petrakou, J. f. Tsai, Y. M. Tzeng

Department of Physics, Faculty of Science, Chulalongkorn University, Bangkok, Thailand

B. Asavapibhop, K. Kovitanggoon, G. Singh, N. Srimanobhas, N. Suwonjandee

Cukurova University, Adana, Turkey

A. Adiguzel, M. N. Bakirci ${ }^{49}$, S. Cerci ${ }^{50}$, Z. S. Demiroglu, C. Dozen, E. Eskut, F. H. Gecit, S. Girgis, G. Gokbulut, Y. Guler, E. Gurpinar, I. Hos, E. E. Kangal ${ }^{51}$, G. Onengut ${ }^{52}$, M. Ozcan, K. Ozdemir ${ }^{53}$, A. Polatoz, D. Sunar $\mathrm{Cerci}^{50}$, H. Topakli ${ }^{49}$, M. Vergili, C. Zorbilmez

Physics Department, Middle East Technical University, Ankara, Turkey

I. V. Akin, B. Bilin, S. Bilmis, B. Isildak ${ }^{54}$, G. Karapinar ${ }^{55}$, M. Yalvac, M. Zeyrek

Bogazici University, Istanbul, Turkey

E. Gülmez, M. Kaya ${ }^{56}$, O. Kaya ${ }^{57}$, E. A. Yetkin ${ }^{58}$, T. Yetkin ${ }^{59}$ 
Istanbul Technical University, Istanbul, Turkey

A. Cakir, K. Cankocak, S. Sen ${ }^{60}$, F. I. Vardarlı

Institute for Scintillation Materials of National Academy of Science of Ukraine, Kharkov, Ukraine

B. Grynyov

National Scientific Center, Kharkov Institute of Physics and Technology, Kharkov, Ukraine

L. Levchuk, P. Sorokin

University of Bristol, Bristol, UK

R. Aggleton, F. Ball, L. Beck, J. J. Brooke, E. Clement, D. Cussans, H. Flacher, J. Goldstein, M. Grimes, G. P. Heath, H. F. Heath, J. Jacob, L. Kreczko, C. Lucas, Z. Meng, D. M. Newbold ${ }^{61}$, S. Paramesvaran, A. Poll, T. Sakuma,

S. Seif El Nasr-storey, S. Senkin, D. Smith, V. J. Smith

Rutherford Appleton Laboratory, Didcot, UK

K. W. Bell, A. Belyaev ${ }^{62}$, C. Brew, R. M. Brown, L. Calligaris, D. Cieri, D. J. A. Cockerill, J. A. Coughlan, K. Harder, S. Harper, E. Olaiya, D. Petyt, C. H. Shepherd-Themistocleous, A. Thea, I. R. Tomalin, T. Williams, S. D. Worm

Imperial College, London, UK

M. Baber, R. Bainbridge, O. Buchmuller, A. Bundock, D. Burton, S. Casasso, M. Citron, D. Colling, L. Corpe, N. Cripps, P. Dauncey, G. Davies, A. De Wit, M. Della Negra, P. Dunne, A. Elwood, W. Ferguson, D. Futyan, G. Hall, G. Iles, M. Kenzie, R. Lane, R. Lucas ${ }^{61}$, L. Lyons, A.-M. Magnan, S. Malik, J. Nash, A. Nikitenko ${ }^{47}$, J. Pela, M. Pesaresi, K. Petridis, D. M. Raymond, A. Richards, A. Rose, C. Seez, A. Tapper, K. Uchida, M. Vazquez Acosta ${ }^{63}$, T. Virdee, S. C. Zenz

Brunel University, Uxbridge, UK

J. E. Cole, P. R. Hobson, A. Khan, P. Kyberd, D. Leggat, D. Leslie, I. D. Reid, P. Symonds, L. Teodorescu, M. Turner

Baylor University, Waco, USA

A. Borzou, K. Call, J. Dittmann, K. Hatakeyama, H. Liu, N. Pastika

The University of Alabama, Tuscaloosa, USA

O. Charaf, S. I. Cooper, C. Henderson, P. Rumerio

Boston University, Boston, USA

D. Arcaro, A. Avetisyan, T. Bose, C. Fantasia, D. Gastler, P. Lawson, D. Rankin, C. Richardson, J. Rohlf, J. St. John, L. Sulak, D. Zou

\section{Brown University, Providence, USA}

J. Alimena, E. Berry, S. Bhattacharya, D. Cutts, N. Dhingra, A. Ferapontov, A. Garabedian, J. Hakala, U. Heintz, E. Laird, G. Landsberg, Z. Mao, M. Narain, S. Piperov, S. Sagir, R. Syarif

University of California, Davis, Davis, USA

R. Breedon, G. Breto, M. Calderon De La Barca Sanchez, S. Chauhan, M. Chertok, J. Conway, R. Conway, P. T. Cox,

R. Erbacher, G. Funk, M. Gardner, W. Ko, R. Lander, M. Mulhearn, D. Pellett, J. Pilot, F. Ricci-Tam, S. Shalhout, J. Smith, M. Squires, D. Stolp, M. Tripathi, S. Wilbur, R. Yohay

University of California, Los Angeles, USA

C. Bravo, R. Cousins, P. Everaerts, C. Farrell, A. Florent, J. Hauser, M. Ignatenko, D. Saltzberg, C. Schnaible, E. Takasugi, V. Valuev, M. Weber

University of California, Riverside, Riverside, USA

K. Burt, R. Clare, J. Ellison, J. W. Gary, G. Hanson, J. Heilman, M. Ivova PANEVA, P. Jandir, E. Kennedy, F. Lacroix, O. R. Long, A. Luthra, M. Malberti, M. Olmedo Negrete, A. Shrinivas, H. Wei, S. Wimpenny, B. R. Yates

University of California, San Diego, La Jolla, USA

J. G. Branson, G. B. Cerati, S. Cittolin, R. T. D’Agnolo, M. Derdzinski, A. Holzner, R. Kelley, D. Klein, J. Letts,

I. Macneill, D. Olivito, S. Padhi, M. Pieri, M. Sani, V. Sharma, S. Simon, M. Tadel, A. Vartak, S. Wasserbaech ${ }^{64}$, C. Welke,

F. Würthwein, A. Yagil, G. Zevi Della Porta 
University of California, Santa Barbara, Santa Barbara, USA

J. Bradmiller-Feld, C. Campagnari, A. Dishaw, V. Dutta, K. Flowers, M. Franco Sevilla, P. Geffert, C. George, F. Golf,

L. Gouskos, J. GranJ. Incandela, N. Mccoll, S. D. Mullin, J. Richman, D. Stuart, I. Suarez, C. West, J. Yoo

California Institute of Technology, Pasadena, USA

D. Anderson, A. Apresyan, A. Bornheim, J. Bunn, Y. Chen, J. Duarte, A. Mott, H. B. Newman, C. Pena, M. Pierini,

M. Spiropulu, J. R. Vlimant, S. Xie, R. Y. Zhu

Carnegie Mellon University, Pittsburgh, USA

M. B. Andrews, V. Azzolini, A. Calamba, B. Carlson, T. Ferguson, M. Paulini, J. Russ, M. Sun, H. Vogel, I. Vorobiev

University of Colorado Boulder, Boulder, USA

J. P. Cumalat, W. T. Ford, A. Gaz, F. Jensen, A. Johnson, M. Krohn, T. Mulholland, U. Nauenberg, K. Stenson,

S. R. Wagner

\section{Cornell University, Ithaca, USA}

J. Alexander, A. Chatterjee, J. Chaves, J. Chu, S. Dittmer, N. Eggert, N. Mirman, G. Nicolas Kaufman, J. R. Patterson, A. Rinkevicius, A. Ryd, L. Skinnari, L. Soffi, W. Sun, S. M. Tan, W. D. Teo, J. Thom, J. Thompson, J. Tucker, Y. Weng, P. Wittich

\section{Fermi National Accelerator Laboratory, Batavia, USA}

S. Abdullin, M. Albrow, G. Apollinari, S. Banerjee, L. A. T. Bauerdick, A. Beretvas, J. Berryhill, P. C. Bhat, G. Bolla, K. Burkett, J. N. Butler, H. W. K. Cheung, F. Chlebana, S. Cihangir, V. D. Elvira, I. Fisk, J. Freeman, E. Gottschalk, L. Gray, D. Green, S. Grünendahl, O. Gutsche, J. Hanlon, D. Hare, R. M. Harris, S. Hasegawa, J. Hirschauer, Z. Hu, B. Jayatilaka, S. Jindariani, M. Johnson, U. Joshi, A. W. Jung, B. Klima, B. Kreis, S. Lammel, J. Linacre, D. Lincoln, R. Lipton, T. Liu, R. Lopes De Sá, J. Lykken, K. Maeshima, J. M. Marraffino, V. I. Martinez Outschoorn, S. Maruyama, D. Mason, P. McBride, P. Merkel, K. Mishra, S. Mrenna, S. Nahn, C. Newman-Holmes ${ }^{\dagger}$, V. O’Dell, K. Pedro, O. Prokofyev, G. Rakness, E. Sexton-Kennedy, A. Soha, W. J. Spalding, L. Spiegel, N. Strobbe, L. Taylor, S. Tkaczyk, N. V. Tran, L. Uplegger, E. W. Vaandering, C. Vernieri, M. Verzocchi, R. Vidal, H. A. Weber, A. Whitbeck

\section{University of Florida, Gainesville, USA}

D. Acosta, P. Avery, P. Bortignon, D. Bourilkov, A. Carnes, M. Carver, D. Curry, S. Das, R. D. Field, I. K. Furic, S. V. Gleyzer, J. Hugon, J. Konigsberg, A. Korytov, K. Kotov, J. F. Low, P. Ma, K. Matchev, H. Mei, P. Milenovic ${ }^{65}$, G. Mitselmakher, D. Rank, R. Rossin, L. Shchutska, M. Snowball, D. Sperka, N. Terentyev, L. Thomas, J. Wang, S. Wang, J. Yelton

\section{Florida International University, Miami, USA}

S. Hewamanage, S. Linn, P. Markowitz, G. Martinez, J. L. Rodriguez

\section{Florida State University, Tallahassee, USA}

A. Ackert, J. R. Adams, T. Adams, A. Askew, S. Bein, J. Bochenek, B. Diamond, J. Haas, S. Hagopian, V. Hagopian, K. F. Johnson, A. Khatiwada, H. Prosper, M. Weinberg

Florida Institute of Technology, Melbourne, USA

M. M. Baarmand, V. Bhopatkar, S. Colafranceschi6 ${ }^{66}$, M. Hohlmann, H. Kalakhety, D. Noonan, T. Roy, F. Yumiceva

University of Illinois at Chicago (UIC), Chicago, USA

M. R. Adams, L. Apanasevich, D. Berry, R. R. Betts, I. Bucinskaite, R. Cavanaugh, O. Evdokimov, L. Gauthier, C. E. Gerber, D. J. Hofman, P. Kurt, C. O’Brien, I. D. Sandoval Gonzalez, C. Silkworth, P. Turner, N. Varelas, Z. Wu, M. Zakaria

\section{The University of Iowa, Iowa City, USA}

B. Bilki ${ }^{67}$, W. Clarida, K. Dilsiz, S. Durgut, R. P. Gandrajula, M. Haytmyradov, V. Khristenko, J.-P. Merlo, H. Mermerkaya ${ }^{68}$, A. Mestvirishvili, A. Moeller, J. Nachtman, H. Ogul, Y. Onel, F. Ozok ${ }^{58}$, A. Penzo, C. Snyder, E. Tiras, J. Wetzel, K. Yi

\section{Johns Hopkins University, Baltimore, USA}

I. Anderson, B. A. Barnett, B. Blumenfeld, N. Eminizer, D. Fehling, L. Feng, A. V. Gritsan, P. Maksimovic, C. Martin, M. Osherson, J. Roskes, A. Sady, U. Sarica, M. Swartz, M. Xiao, Y. Xin, C. You 
The University of Kansas, Lawrence, USA

P. Baringer, A. Bean, G. Benelli, C. Bruner, R. P. KennyIII, D. Majumder, M. Malek, M. Murray, S. Sanders, R. Stringer, Q. Wang

\section{Kansas State University, Manhattan, USA}

A. Ivanov, K. Kaadze, S. Khalil, M. Makouski, Y. Maravin, A. Mohammadi, L. K. Saini, N. Skhirtladze, S. Toda

\section{Lawrence Livermore National Laboratory, Livermore, USA}

D. Lange, F. Rebassoo, D. Wright

\section{University of Maryland, College Park, USA}

C. Anelli, A. Baden, O. Baron, A. Belloni, B. Calvert, S. C. Eno, C. Ferraioli, J. A. Gomez, N. J. Hadley, S. Jabeen, R. G. Kellogg, T. Kolberg, J. Kunkle, Y. Lu, A. C. Mignerey, Y. H. Shin, A. Skuja, M. B. Tonjes, S. C. Tonwar

Massachusetts Institute of Technology, Cambridge, USA

A. Apyan, R. Barbieri, A. Baty, K. Bierwagen, S. Brandt, W. Busza, I. A. Cali, Z. Demiragli, L. Di Matteo,

G. Gomez Ceballos, M. Goncharov, D. Gulhan, Y. Iiyama, G. M. Innocenti, M. Klute, D. Kovalskyi, Y. S. Lai, Y.-J. Lee,

A. Levin, P. D. Luckey, A. C. Marini, C. Mcginn, C. Mironov, S. Narayanan, X. Niu, C. Paus, D. Ralph, C. Roland,

G. Roland, J. Salfeld-Nebgen, G. S. F. Stephans, K. Sumorok, M. Varma, D. Velicanu, J. Veverka, J. Wang, T. W. Wang,

B. Wyslouch, M. Yang, V. Zhukova

University of Minnesota, Minneapolis, USA

B. Dahmes, A. Evans, A. Finkel, A. Gude, P. Hansen, S. Kalafut, S. C. Kao, K. Klapoetke, Y. Kubota, Z. Lesko, J. Mans, S. Nourbakhsh, N. Ruckstuhl, R. Rusack, N. Tambe, J. Turkewitz

University of Mississippi, Oxford, USA

J. G. Acosta, S. Oliveros

University of Nebraska-Lincoln, Lincoln, USA

E. Avdeeva, K. Bloom, S. Bose, D. R. Claes, A. Dominguez, C. Fangmeier, R. Gonzalez Suarez, R. Kamalieddin, J. Keller, D. Knowlton, I. Kravchenko, F. Meier, J. Monroy, F. Ratnikov, J. E. Siado, G. R. Snow

\section{State University of New York at Buffalo, Buffalo, USA}

M. Alyari, J. Dolen, J. George, A. Godshalk, C. Harrington, I. Iashvili, J. Kaisen, A. Kharchilava, A. Kumar, S. Rappoccio, B. Roozbahani

\section{Northeastern University, Boston, USA}

G. Alverson, E. Barberis, D. Baumgartel, M. Chasco, A. Hortiangtham, A. Massironi, D. M. Morse, D. Nash, T. Orimoto,

R. Teixeira De Lima, D. Trocino, R.-J. Wang, D. Wood, J. Zhang

\section{Northwestern University, Evanston, USA}

K. A. Hahn, A. Kubik, N. Mucia, N. Odell, B. Pollack, A. Pozdnyakov, M. Schmitt, S. Stoynev, K. Sung, M. Trovato, M. Velasco

\section{University of Notre Dame, Notre Dame, USA}

A. Brinkerhoff, N. Dev, M. Hildreth, C. Jessop, D. J. Karmgard, N. Kellams, K. Lannon, N. Marinelli, F. Meng, C. Mueller, Y. Musienko ${ }^{38}$, M. Planer, A. Reinsvold, R. Ruchti, G. Smith, S. Taroni, N. Valls, M. Wayne, M. Wolf, A. Woodard

The Ohio State University, Columbus, USA

L. Antonelli, J. Brinson, B. Bylsma, L. S. Durkin, S. Flowers, A. Hart, C. Hill, R. Hughes, W. Ji, T. Y. Ling, B. Liu, W. Luo, D. Puigh, M. Rodenburg, B. L. Winer, H. W. Wulsin

Princeton University, Princeton, USA

O. Driga, P. Elmer, J. Hardenbrook, P. Hebda, S. A. Koay, P. Lujan, D. Marlow, T. Medvedeva, M. Mooney, J. Olsen,

C. Palmer, P. Piroué, H. Saka, D. Stickland, C. Tully, A. Zuranski

\section{University of Puerto Rico, Mayagüez, USA}

S. Malik 
Purdue University, West Lafayette, USA

V. E. Barnes, D. Benedetti, D. Bortoletto, L. Gutay, M. K. Jha, M. Jones, K. Jung, D. H. Miller, N. Neumeister,

B. C. Radburn-Smith, X. Shi, I. Shipsey, D. Silvers, J. Sun, A. Svyatkovskiy, F. Wang, W. Xie, L. Xu

Purdue University Calumet, Hammond, USA

N. Parashar, J. Stupak

Rice University, Houston, USA

A. Adair, B. Akgun, Z. Chen, K. M. Ecklund, F. J. M. Geurts, M. Guilbaud, W. Li, B. Michlin, M. Northup, B. P. Padley, R. Redjimi, J. Roberts, J. Rorie, Z. Tu, J. Zabel

University of Rochester, Rochester, USA

B. Betchart, A. Bodek, P. de Barbaro, R. Demina, Y. Eshaq, T. Ferbel, M. Galanti, A. Garcia-Bellido, J. Han, A. Harel, O. Hindrichs, A. Khukhunaishvili, G. Petrillo, P. Tan, M. Verzetti

Rutgers, The State University of New Jersey, Piscataway, USA

S. Arora, A. Barker, J. P. Chou, C. Contreras-Campana, E. Contreras-Campana, D. Ferencek, Y. Gershtein, R. Gray, E. Halkiadakis, D. Hidas, E. Hughes, S. Kaplan, R. Kunnawalkam Elayavalli, A. Lath, K. Nash, S. Panwalkar, M. Park, S. Salur, S. Schnetzer, D. Sheffield, S. Somalwar, R. Stone, S. Thomas, P. Thomassen, M. Walker

University of Tennessee, Knoxville, USA

M. Foerster, G. Riley, K. Rose, S. Spanier, A. York

Texas A\&M University, College Station, USA

O. Bouhali ${ }^{69}$, A. Castaneda Hernandez ${ }^{69}$, A. Celik, M. Dalchenko, M. De Mattia, A. Delgado, S. Dildick, R. Eusebi,

J. Gilmore, T. Huang, T. Kamon ${ }^{70}$, V. Krutelyov, V. Krutelyov, R. Mueller, I. Osipenkov, Y. Pakhotin, R. Patel, A. Perloff, A. Rose, A. Safonov, A. Tatarinov, K. A. Ulmer ${ }^{2}$

Texas Tech University, Lubbock, USA

N. Akchurin, C. Cowden, J. Damgov, C. Dragoiu, P. R. Dudero, J. Faulkner, S. Kunori, K. Lamichhane, S. W. Lee, T. Libeiro, S. Undleeb, I. Volobouev

\section{Vanderbilt University, Nashville, USA}

E. Appelt, A. G. Delannoy, S. Greene, A. Gurrola, R. Janjam, W. Johns, C. Maguire, Y. Mao, A. Melo, H. Ni, P. Sheldon,

B. Snook, S. Tuo, J. Velkovska, Q. Xu

\section{University of Virginia, Charlottesville, USA}

M. W. Arenton, B. Cox, B. Francis, J. Goodell, R. Hirosky, A. Ledovskoy, H. Li, C. Lin, C. Neu, T. Sinthuprasith, X. Sun, Y. Wang, E. Wolfe, J. Wood, F. Xia

\section{Wayne State University, Detroit, USA}

C. Clarke, R. Harr, P. E. Karchin, C. Kottachchi Kankanamge Don, P. Lamichhane, J. Sturdy

\section{University of Wisconsin, Madison, USA}

D. A. Belknap, D. Carlsmith, M. Cepeda, S. Dasu, L. Dodd, S. Duric, B. Gomber, R. Hall-Wilton, M. Herndon, A. Hervé, P. Klabbers, A. Lanaro, A. Levine, K. Long, R. Loveless, A. Mohapatra, I. Ojalvo, T. Perry, G. A. Pierro, G. Polese,

T. Ruggles, T. Sarangi, A. Savin, A. Sharma, N. Smith, W. H. Smith, D. Taylor, N. Woods

\section{$\dagger$ Deceased}

1: Also at Vienna University of Technology, Vienna, Austria

2: Also at CERN, European Organization for Nuclear Research, Geneva, Switzerland

3: Also at State Key Laboratory of Nuclear Physics and Technology, Peking University, Beijing, China

4: Also at Institut Pluridisciplinaire Hubert Curien, Université de Strasbourg, Université de Haute Alsace Mulhouse, CNRS/IN2P3, Strasbourg, France

5: Also at National Institute of Chemical Physics and Biophysics, Tallinn, Estonia

6: Also at Skobeltsyn Institute of Nuclear Physics, Lomonosov Moscow State University, Moscow, Russia

7: Also at Universidade Estadual de Campinas, Campinas, Brazil

8: Also at Centre National de la Recherche Scientifique (CNRS)-IN2P3, Paris, France 
9: Also at Laboratoire Leprince-Ringuet, Ecole Polytechnique, IN2P3-CNRS, Palaiseau, France

10: Also at Joint Institute for Nuclear Research, Dubna, Russia

11: Also at Helwan University, Cairo, Egypt

12: Now at Zewail City of Science and Technology, Zewail, Egypt

13: Now at Fayoum University, El-Fayoum, Egypt

14: Also at British University in Egypt, Cairo, Egypt

15: Now at Ain Shams University, Cairo, Egypt

16: Also at Université de Haute Alsace, Mulhouse, France

17: Also at Tbilisi State University, Tbilisi, Georgia

18: Also at RWTH Aachen University, III. Physikalisches Institut A, Aachen, Germany

19: Also at Indian Institute of Science Education and Research, Bhopal, India

20: Also at University of Hamburg, Hamburg, Germany

21: Also at Brandenburg University of Technology, Cottbus, Germany

22: Also at Institute of Nuclear Research ATOMKI, Debrecen, Hungary

23: Also at Eötvös Loránd University, Budapest, Hungary

24: Also at University of Debrecen, Debrecen, Hungary

25: Also at Wigner Research Centre for Physics, Budapest, Hungary

26: Also at University of Visva-Bharati, Santiniketan, India

27: Now at King Abdulaziz University, Jeddah, Saudi Arabia

28: Also at University of Ruhuna, Matara, Sri Lanka

29: Also at Isfahan University of Technology, Isfahan, Iran

30: Also at University of Tehran, Department of Engineering Science, Tehran, Iran

31: Also at Plasma Physics Research Center, Science and Research Branch, Islamic Azad University, Tehran, Iran

32: Also at Università degli Studi di Siena, Siena, Italy

33: Also at Purdue University, West Lafayette, USA

34: Also at International Islamic University of Malaysia, Kuala Lumpur, Malaysia

35: Also at Malaysian Nuclear Agency, MOSTI, Kajang, Malaysia

36: Also at Consejo Nacional de Ciencia y Tecnología, Mexico City, Mexico

37: Also at Warsaw University of Technology, Institute of Electronic Systems, Warsaw, Poland

38: Also at Institute for Nuclear Research, Moscow, Russia

39: Now at National Research Nuclear University 'Moscow Engineering Physics Institute' (MEPhI), Moscow, Russia

40: Also at St. Petersburg State Polytechnical University, St. Petersburg, Russia

41: Also at California Institute of Technology, Pasadena, USA

42: Also at Faculty of Physics, University of Belgrade, Belgrade, Serbia

43: Also at INFN Sezione di Roma; Università di Roma, Rome, Italy

44: Also at National Technical University of Athens, Athens, Greece

45: Also at Scuola Normale e Sezione dell'INFN, Pisa, Italy

46: Also at University of Athens, Athens, Greece

47: Also at Institute for Theoretical and Experimental Physics, Moscow, Russia

48: Also at Albert Einstein Center for Fundamental Physics, Bern, Switzerland

49: Also at Gaziosmanpasa University, Tokat, Turkey

50: Also at Adiyaman University, Adiyaman, Turkey

51: Also at Mersin University, Mersin, Turkey

52: Also at Cag University, Mersin, Turkey

53: Also at Piri Reis University, Istanbul, Turkey

54: Also at Ozyegin University, Istanbul, Turkey

55: Also at Izmir Institute of Technology, Izmir, Turkey

56: Also at Marmara University, Istanbul, Turkey

57: Also at Kafkas University, Kars, Turkey

58: Also at Mimar Sinan University, Istanbul, Istanbul, Turkey

59: Also at Yildiz Technical University, Istanbul, Turkey

60: Also at Hacettepe University, Ankara, Turkey

61: Also at Rutherford Appleton Laboratory, Didcot, UK 
62: Also at School of Physics and Astronomy, University of Southampton, Southampton, UK

63: Also at Instituto de Astrofísica de Canarias, La Laguna, Spain

64: Also at Utah Valley University, Orem, USA

65: Also at University of Belgrade, Faculty of Physics and Vinca Institute of Nuclear Sciences, Belgrade, Serbia

66: Also at Facoltà Ingegneria, Università di Roma, Rome, Italy

67: Also at Argonne National Laboratory, Argonne, USA

68: Also at Erzincan University, Erzincan, Turkey

69: Also at Texas A\&M University at Qatar, Doha, Qatar

70: Also at Kyungpook National University, Daegu, Korea 